BULLETIN (New Series) OF THE

AMERICAN MATHEMATICAL SOCIETY

Volume 39, Number 2, Pages 145-205

S 0273-0979(01)00934-X

Article electronically published on December 21, 2001

\title{
THE OCTONIONS
}

\author{
JOHN C. BAEZ
}

\begin{abstract}
The octonions are the largest of the four normed division algebras. While somewhat neglected due to their nonassociativity, they stand at the crossroads of many interesting fields of mathematics. Here we describe them and their relation to Clifford algebras and spinors, Bott periodicity, projective and Lorentzian geometry, Jordan algebras, and the exceptional Lie groups. We also touch upon their applications in quantum logic, special relativity and supersymmetry.
\end{abstract}

\section{INTRODUCTION}

There are exactly four normed division algebras: the real numbers $(\mathbb{R})$, complex numbers $(\mathbb{C})$, quaternions $(\mathbb{H})$, and octonions $(\mathbb{O})$. The real numbers are the dependable breadwinner of the family, the complete ordered field we all rely on. The complex numbers are a slightly flashier but still respectable younger brother: not ordered, but algebraically complete. The quaternions, being noncommutative, are the eccentric cousin who is shunned at important family gatherings. But the octonions are the crazy old uncle nobody lets out of the attic: they are nonassociative.

Most mathematicians have heard the story of how Hamilton invented the quaternions. In 1835, at the age of 30 , he had discovered how to treat complex numbers as pairs of real numbers. Fascinated by the relation between $\mathbb{C}$ and 2-dimensional geometry, he tried for many years to invent a bigger algebra that would play a similar role in 3-dimensional geometry. In modern language, it seems he was looking for a 3dimensional normed division algebra. His quest built to its climax in October 1843. He later wrote to his son, "Every morning in the early part of the above-cited month, on my coming down to breakfast, your (then) little brother William Edwin, and yourself, used to ask me: 'Well, Papa, can you multiply triplets?' Whereto I was always obliged to reply, with a sad shake of the head: 'No, I can only add and subtract them.'" The problem, of course, was that there exists no 3-dimensional normed division algebra. He really needed a 4-dimensional algebra.

Finally, on the 16th of October, 1843, while walking with his wife along the Royal Canal to a meeting of the Royal Irish Academy in Dublin, he made his momentous discovery. "That is to say, I then and there felt the galvanic circuit of thought close; and the sparks which fell from it were the fundamental equations between $i, j, k$; exactly such as I have used them ever since." And in a famous act of mathematical vandalism, he carved these equations into the stone of the Brougham Bridge:

$$
i^{2}=j^{2}=k^{2}=i j k=-1 .
$$

Received by the editors May 31, 2001, and in revised form August 2, 2001.

2000 Mathematics Subject Classification. Primary 17-02, 17A35, 17C40, 17C90, 22E70. 
One reason this story is so well-known is that Hamilton spent the rest of his life obsessed with the quaternions and their applications to geometry [44], [52]. And for a while, quaternions were fashionable. They were made a mandatory examination topic in Dublin, and in some American universities they were the only advanced mathematics taught. Much of what we now do with scalars and vectors in $\mathbb{R}^{3}$ was then done using real and imaginary quaternions. A school of 'quaternionists' developed, which was led after Hamilton's death by Peter Tait of Edinburgh and Benjamin Peirce of Harvard. Tait wrote 8 books on the quaternions, emphasizing their applications to physics. When Gibbs invented the modern notation for the dot product and cross product, Tait condemned it as a "hermaphrodite monstrosity". A war of polemics ensued, with such luminaries as Kelvin and Heaviside weighing in on the side of vectors. Ultimately the quaternions lost and acquired a slight taint of disgrace from which they have never fully recovered [26].

Less well-known is the discovery of the octonions by Hamilton's friend from college, John T. Graves. It was Graves' interest in algebra that got Hamilton thinking about complex numbers and triplets in the first place. The very day after his fateful walk, Hamilton sent an 8-page letter describing the quaternions to Graves. Graves replied on October 26th, complimenting Hamilton on the boldness of the idea, but adding "There is still something in the system which gravels me. I have not yet any clear views as to the extent to which we are at liberty arbitrarily to create imaginaries, and to endow them with supernatural properties." And he asked: "If with your alchemy you can make three pounds of gold, why should you stop there?"

Graves then set to work on some gold of his own! On December 26th, he wrote to Hamilton describing a new 8-dimensional algebra, which he called the 'octaves'. He showed that they were a normed division algebra and used this to express the product of two sums of eight perfect squares as another sum of eight perfect squares: the 'eight squares theorem' [51].

In January 1844, Graves sent three letters to Hamilton expanding on his discovery. He considered the idea of a general theory of ' $2^{m}$-ions' and tried to construct a 16-dimensional normed division algebra, but he "met with an unexpected hitch" and came to doubt that this was possible. Hamilton offered to publicize Graves' discovery, but being busy with work on quaternions, he kept putting it off. In July he wrote to Graves pointing out that the octonions were nonassociative: " $A \cdot B C=A B \cdot C=A B C$, if $A, B, C$ be quaternions, but not so, generally, with your octaves." In fact, Hamilton first invented the term 'associative' at about this time, so the octonions may have played a role in clarifying the importance of this concept.

Meanwhile the young Arthur Cayley, fresh out of Cambridge, had been thinking about the quaternions ever since Hamilton announced their existence. He seemed to be seeking relationships between the quaternions and hyperelliptic functions. In March of 1845, he published a paper in the Philosophical Magazine entitled 'On Jacobi's Elliptic Functions, in Reply to the Rev. B. Bronwin; and on Quaternions' 17]. The bulk of this paper was an attempt to rebut an article pointing out mistakes in Cayley's work on elliptic functions. Apparently as an afterthought, he tacked on a brief description of the octonions. In fact, this paper was so full of errors that it was omitted from his collected works - except for the part about octonions [18].

Upset at being beaten to publication, Graves attached a postscript to a paper of his own which was to appear in the following issue of the same journal, saying that 
he had known of the octonions ever since Christmas, 1843. On June 14th, 1847, Hamilton contributed a short note to the Transactions of the Royal Irish Academy, vouching for Graves' priority. But it was too late: the octonions became known as 'Cayley numbers'. Still worse, Graves later found that his eight squares theorem had already been discovered by C. F. Degen in 1818 [27], 29.

Why have the octonions languished in such obscurity compared to the quaternions? Besides their rather inglorious birth, one reason is that they lacked a tireless defender such as Hamilton. But surely the reason for this is that they lacked any clear application to geometry and physics. The unit quaternions form the group $\mathrm{SU}(2)$, which is the double cover of the rotation group $\mathrm{SO}(3)$. This makes them nicely suited to the study of rotations and angular momentum, particularly in the context of quantum mechanics. These days we regard this phenomenon as a special case of the theory of Clifford algebras. Most of us no longer attribute to the quaternions the cosmic significance that Hamilton claimed for them, but they fit nicely into our understanding of the scheme of things.

The octonions, on the other hand, do not. Their relevance to geometry was quite obscure until 1925, when Élie Cartan described 'triality' - the symmetry between vectors and spinors in 8-dimensional Euclidean space [16]. Their potential relevance to physics was noticed in a 1934 paper by Jordan, von Neumann and Wigner on the foundations of quantum mechanics [58]. However, attempts by Jordan and others to apply octonionic quantum mechanics to nuclear and particle physics met with little success. Work along these lines continued quite slowly until the 1980s, when it was realized that the octonions explain some curious features of string theory [63]. The Lagrangian for the classical superstring involves a relationship between vectors and spinors in Minkowski spacetime which holds only in 3, 4, 6, and 10 dimensions. Note that these numbers are 2 more than the dimensions of $\mathbb{R}, \mathbb{C}, \mathbb{H}$ and $\mathbb{O}$. As we shall see, this is no coincidence: briefly, the isomorphisms

$$
\begin{aligned}
& \mathfrak{s l}(2, \mathbb{R}) \cong \mathfrak{s o}(2,1) \\
& \mathfrak{s l}(2, \mathbb{C}) \cong \mathfrak{s o}(3,1) \\
& \mathfrak{s l}(2, \mathbb{H}) \cong \mathfrak{s o}(5,1) \\
& \mathfrak{s l}(2, \mathbb{O}) \cong \mathfrak{s o}(9,1)
\end{aligned}
$$

allow us to treat a spinor in one of these dimensions as a pair of elements of the corresponding division algebra. It is fascinating that of these superstring Lagrangians, it is the 10-dimensional octonionic one that gives the most promising candidate for a realistic theory of fundamental physics! However, there is still no proof that the octonions are useful for understanding the real world. We can only hope that eventually this question will be settled one way or another.

Besides their possible role in physics, the octonions are important because they tie together some algebraic structures that otherwise appear as isolated and inexplicable exceptions. As we shall explain, the concept of an octonionic projective space $\mathbb{O} \mathbb{P}^{n}$ only makes sense for $n \leq 2$, due to the nonassociativity of $\mathbb{O}$. This means that various structures associated to real, complex and quaternionic projective spaces have octonionic analogues only for $n \leq 2$.

Simple Lie algebras are a nice example of this phenomenon. There are 3 infinite families of 'classical' simple Lie algebras, which come from the isometry groups of the projective spaces $\mathbb{R} \mathbb{P}^{n}, \mathbb{C P}^{n}$ and $\mathbb{H} \mathbb{P}^{n}$. There are also 5 'exceptional' simple Lie algebras. These were discovered by Killing and Cartan in the late 1800s. At the time, the significance of these exceptions was shrouded in mystery: they did not 
arise as symmetry groups of known structures. Only later did their connection to the octonions become clear. It turns out that 4 of them come from the isometry groups of the projective planes over $\mathbb{O}, \mathbb{O} \otimes \mathbb{C}, \mathbb{O} \otimes \mathbb{H}$ and $\mathbb{O} \otimes \mathbb{O}$. The remaining one is the automorphism group of the octonions!

Another good example is the classification of simple formally real Jordan algebras. Besides several infinite families of these, there is the 'exceptional' Jordan algebra, which consists of $3 \times 3$ hermitian octonionic matrices. Minimal projections in this Jordan algebra correspond to points of $\mathbb{O P}^{2}$, and the automorphism group of this algebra is the same as the isometry group of $\mathbb{O P}^{2}$.

The octonions also have fascinating connections to topology. In 1957, Raoul Bott computed the homotopy groups of the topological group $\mathrm{O}(\infty)$, which is the inductive limit of the orthogonal groups $\mathrm{O}(n)$ as $n \rightarrow \infty$. He proved that they repeat with period 8 :

$$
\pi_{i+8}(\mathrm{O}(\infty)) \cong \pi_{i}(\mathrm{O}(\infty))
$$

This is known as 'Bott periodicity'. He also computed the first 8:

$$
\begin{aligned}
& \pi_{0}(\mathrm{O}(\infty)) \cong \mathbb{Z}_{2} \\
& \pi_{1}(\mathrm{O}(\infty)) \cong \mathbb{Z}_{2} \\
& \pi_{2}(\mathrm{O}(\infty)) \cong 0 \\
& \pi_{3}(\mathrm{O}(\infty)) \cong \mathbb{Z} \\
& \pi_{4}(\mathrm{O}(\infty)) \cong 0 \\
& \pi_{5}(\mathrm{O}(\infty)) \cong 0 \\
& \pi_{6}(\mathrm{O}(\infty)) \cong 0 \\
& \pi_{7}(\mathrm{O}(\infty)) \cong \mathbb{Z}
\end{aligned}
$$

Note that the nonvanishing homotopy groups here occur in dimensions one less than the dimensions of $\mathbb{R}, \mathbb{C}, \mathbb{H}$, and $\mathbb{O}$. This is no coincidence! In a normed division algebra, left multiplication by an element of norm one defines an orthogonal transformation of the algebra, and thus an element of $\mathrm{O}(\infty)$. This gives us maps from the spheres $S^{0}, S^{1}, S^{3}$ and $S^{7}$ to $\mathrm{O}(\infty)$, and these maps generate the homotopy groups in those dimensions.

Given this, one might naturally guess that the period- 8 repetition in the homotopy groups of $\mathrm{O}(\infty)$ is in some sense 'caused' by the octonions. As we shall see, this is true. Conversely, Bott periodicity is closely connected to the problem of how many pointwise linearly independent smooth vector fields can be found on the $n$-sphere [55. There exist $n$ such vector fields only when $n+1=1,2,4$, or 8 , and this can be used to show that division algebras over the reals can only occur in these dimensions.

In what follows we shall try to explain the octonions and their role in algebra, geometry, and topology. In Section 2 we give four constructions of the octonions: first via their multiplication table, then using the Fano plane, then using the CayleyDickson construction and finally using Clifford algebras, spinors, and a generalized concept of 'triality' advocated by Frank Adams [2]. Each approach has its own merits. In Section 3 we discuss the projective lines and planes over the normed division algebras - especially $\mathbb{O}$ - and describe their relation to Bott periodicity, the exceptional Jordan algebra, and the Lie algebra isomorphisms listed above. 
Finally, in Section 4 we discuss octonionic constructions of the exceptional Lie groups, especially the 'magic square'.

1.1. Preliminaries. Before our tour begins, let us settle on some definitions. For us a vector space will always be a finite-dimensional module over the field of real numbers. An algebra $A$ will be a vector space that is equipped with a bilinear map $m: A \times A \rightarrow A$ called 'multiplication' and a nonzero element $1 \in A$ called the 'unit' such that $m(1, a)=m(a, 1)=a$. As usual, we abbreviate $m(a, b)$ as $a b$. We do not assume our algebras are associative! Given an algebra, we will freely think of real numbers as elements of this algebra via the map $\alpha \mapsto \alpha 1$.

An algebra $A$ is a division algebra if given $a, b \in A$ with $a b=0$, then either $a=0$ or $b=0$. Equivalently, $A$ is a division algebra if the operations of left and right multiplication by any nonzero element are invertible. A normed division algebra is an algebra $A$ that is also a normed vector space with $\|a b\|=\|a\|\|b\|$. This implies that $A$ is a division algebra and that $\|1\|=1$.

We should warn the reader of some subtleties. We say an algebra $A$ has multiplicative inverses if for any nonzero $a \in A$ there is an element $a^{-1} \in A$ with $a a^{-1}=a^{-1} a=1$. An associative algebra has multiplicative inverses iff it is a division algebra. However, this fails for nonassociative algebras! In Section 2.2 we shall construct algebras that have multiplicative inverses, but are not division algebras. On the other hand, we can construct a division algebra without multiplicative inverses by taking the quaternions and modifying the product slightly, setting $i^{2}=-1+\epsilon j$ for some small nonzero real number $\epsilon$ while leaving the rest of the multiplication table unchanged. The element $i$ then has both right and left inverses, but they are not equal. (We thank David Rusin for this example.)

There are three levels of associativity. An algebra is power-associative if the subalgebra generated by any one element is associative. It is alternative if the subalgebra generated by any two elements is associative. Finally, if the subalgebra generated by any three elements is associative, the algebra is associative.

As we shall see, the octonions are not associative, but they are alternative. How does one check a thing like this? By a theorem of Emil Artin [83], an algebra $A$ is alternative iff for all $a, b \in A$ we have

$$
(a a) b=a(a b), \quad(a b) a=a(b a), \quad(b a) a=b(a a) .
$$

In fact, any two of these equations implies the remaining one, so people usually take the first and last as the definition of 'alternative'. To see this fact, note that any algebra has a trilinear map

$$
[\cdot, \cdot, \cdot]: A^{3} \rightarrow A
$$

called the associator, given by

$$
[a, b, c]=(a b) c-a(b c) .
$$

The associator measures the failure of associativity just as the commutator $[a, b]=$ $a b-b a$ measures the failure of commutativity. Now, the commutator is an alternating bilinear map, meaning that it switches sign whenever the two arguments are exchanged:

$$
[a, b]=-[b, a]
$$

or equivalently, that it vanishes when they are equal:

$$
[a, a]=0 .
$$


This raises the question of whether the associator is alternating too. In fact, this holds precisely when $A$ is alternative! The reason is that each equation in (1) says that the associator vanishes when a certain pair of arguments are equal, or equivalently, that it changes sign when that pair of arguments is switched. Note, however, that if the associator changes sign when we switch the $i$ th and $j$ th arguments, and also when we switch the $j$ th and $k$ th arguments, it must change sign when we switch the $i$ th and $k$ th. Thus any two of equations (1) imply the third.

Now we can say what is so great about $\mathbb{R}, \mathbb{C}, \mathbb{H}$, and $\mathbb{O}$ :

Theorem 1. $\mathbb{R}, \mathbb{C}, \mathbb{H}$, and $\mathbb{O}$ are the only normed division algebras.

Theorem 2. $\mathbb{R}, \mathbb{C}, \mathbb{H}$, and $\mathbb{O}$ are the only alternative division algebras.

The first theorem goes back to an 1898 paper by Hurwitz [54. It was subsequently generalized in many directions, for example, to algebras over other fields. A version of the second theorem appears in an 1930 paper by Zorn [99] - the guy with the lemma. For modern proofs of both these theorems, see Schafer's excellent book on nonassociative algebras [83. We sketch a couple of proofs of Hurwitz's theorem in Section 2.3

Note that we did not state that $\mathbb{R}, \mathbb{C}, \mathbb{H}$ and $\mathbb{O}$ are the only division algebras. This is not true. For example, we have already described a way to get 4-dimensional division algebras that do not have multiplicative inverses. However, we do have this fact:

Theorem 3. All division algebras have dimension 1,2, 4, or 8.

This was independently proved by Kervaire [61] and Bott-Milnor [1] in 1958. We will say a bit about the proof in Section 3.1. However, in what follows our main focus will not be on general results about division algebras. Instead, we concentrate on special features of the octonions. Let us begin by constructing them.

\section{Constructing the octonions}

The most elementary way to construct the octonions is to give their multiplication table. The octonions are an 8-dimensional algebra with basis $1, e_{1}, e_{2}, e_{3}, e_{4}, e_{5}$, $e_{6}, e_{7}$, and their multiplication is given in this table, which describes the result of multiplying the element in the $i$ th row by the element in the $j$ th column:

\begin{tabular}{|c|c|c|c|c|c|c|c|}
\hline & $e_{1}$ & $e_{2}$ & $e_{3}$ & $e_{4}$ & $e_{5}$ & $e_{6}$ & $e_{7}$ \\
\hline$e_{1}$ & -1 & $e_{4}$ & $e_{7}$ & $-e_{2}$ & $e_{6}$ & $-e_{5}$ & $-e_{3}$ \\
\hline$e_{2}$ & $-e_{4}$ & -1 & $e_{5}$ & $e_{1}$ & $-e_{3}$ & $e_{7}$ & $-e_{6}$ \\
\hline$e_{3}$ & $-e_{7}$ & $-e_{5}$ & -1 & $e_{6}$ & $e_{2}$ & $-e_{4}$ & $e_{1}$ \\
\hline$e_{4}$ & $e_{2}$ & $-e_{1}$ & $-e_{6}$ & -1 & $e_{7}$ & $e_{3}$ & $-e_{5}$ \\
\hline$e_{5}$ & $-e_{6}$ & $e_{3}$ & $-e_{2}$ & $-e_{7}$ & -1 & $e_{1}$ & $e_{4}$ \\
\hline$e_{6}$ & $e_{5}$ & $-e_{7}$ & $e_{4}$ & $-e_{3}$ & $-e_{1}$ & -1 & $e_{2}$ \\
\hline$e_{7}$ & $e_{3}$ & $e_{6}$ & $-e_{1}$ & $e_{5}$ & $-e_{4}$ & $-e_{2}$ & -1 \\
\hline
\end{tabular}

TABLE 1. Octonion Multiplication Table

Unfortunately, this table is almost completely unenlightening! About the only interesting things one can easily learn from it are: 
- $e_{1}, \ldots, e_{7}$ are square roots of -1 ,

- $e_{i}$ and $e_{j}$ anticommute when $i \neq j$ :

$$
e_{i} e_{j}=-e_{j} e_{i}
$$

- the index cycling identity holds:

$$
e_{i} e_{j}=e_{k} \Longrightarrow e_{i+1} e_{j+1}=e_{k+1}
$$

where we think of the indices as living in $\mathbb{Z}_{7}$, and

- the index doubling identity holds:

$$
e_{i} e_{j}=e_{k} \Longrightarrow e_{2 i} e_{2 j}=e_{2 k}
$$

Together with a single nontrivial product like $e_{1} e_{2}=e_{4}$, these facts are enough to recover the whole multiplication table. However, we really want a better way to remember the octonion product. We should become as comfortable with multiplying octonions as we are with multiplying matrices! And ultimately, we want a more conceptual approach to the octonions, which explains their special properties and how they fit in with other mathematical ideas. In what follows, we give some more descriptions of octonion multiplication, starting with a nice mnemonic, and working up to some deeper, more conceptual ones.

2.1. The Fano plane. The quaternions, $\mathbb{H}$, are a 4-dimensional algebra with basis $1, i, j, k$. To describe the product we could give a multiplication table, but it is easier to remember that:

- 1 is the multiplicative identity,

- $i, j$, and $k$ are square roots of -1 ,

- we have $i j=k, j i=-k$, and all identities obtained from these by cyclic permutations of $(i, j, k)$.

We can summarize the last rule in a picture:

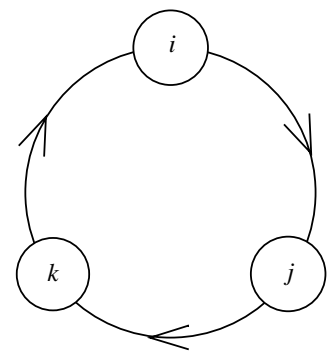

When we multiply two elements going clockwise around the circle, we get the next one: for example, $i j=k$. But when we multiply two going around counterclockwise, we get minus the next one: for example, $j i=-k$.

We can use the same sort of picture to remember how to multiply octonions: 


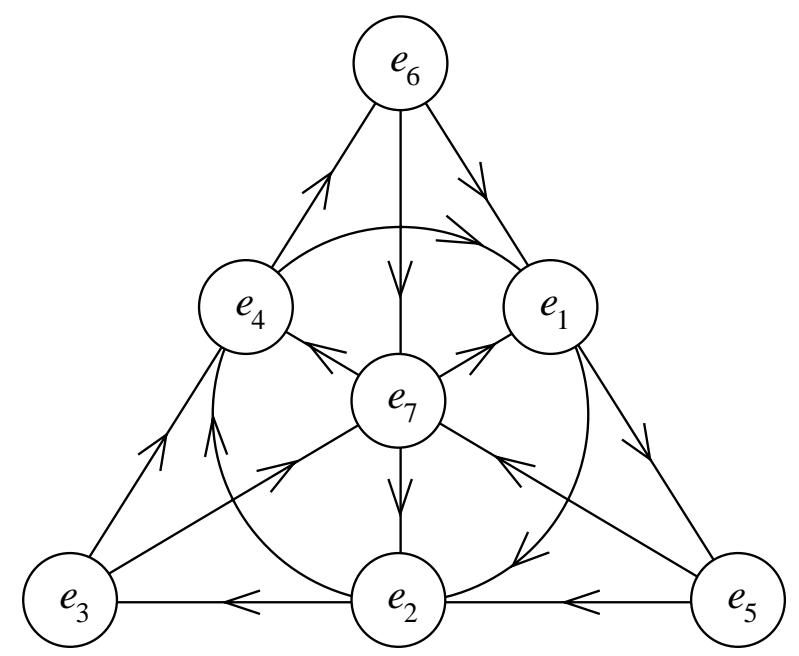

This is the Fano plane, a little gadget with 7 points and 7 lines. The 'lines' are the sides of the triangle, its altitudes, and the circle containing all the midpoints of the sides. Each pair of distinct points lies on a unique line. Each line contains three points, and each of these triples has a cyclic ordering shown by the arrows. If $e_{i}, e_{j}$, and $e_{k}$ are cyclically ordered in this way, then

$$
e_{i} e_{j}=e_{k}, \quad e_{j} e_{i}=-e_{k} .
$$

Together with these rules:

- 1 is the multiplicative identity,

- $e_{1}, \ldots, e_{7}$ are square roots of -1 ,

the Fano plane completely describes the algebra structure of the octonions. Indexdoubling corresponds to rotating the picture a third of a turn.

This is certainly a neat mnemonic, but is there anything deeper lurking behind it? Yes! The Fano plane is the projective plane over the 2-element field $\mathbb{Z}_{2}$. In other words, it consists of lines through the origin in the vector space $\mathbb{Z}_{2}^{3}$. Since every such line contains a single nonzero element, we can also think of the Fano plane as consisting of the seven nonzero elements of $\mathbb{Z}_{2}^{3}$. If we think of the origin in $\mathbb{Z}_{2}^{3}$ as corresponding to $1 \in \mathbb{O}$, we get the following picture of the octonions:

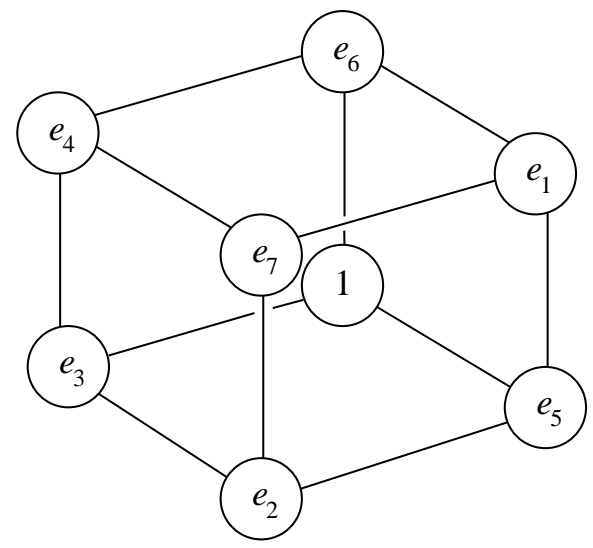


Note that planes through the origin of this 3 -dimensional vector space give subalgebras of $\mathbb{O}$ isomorphic to the quaternions, lines through the origin give subalgebras isomorphic to the complex numbers, and the origin itself gives a subalgebra isomorphic to the real numbers.

What we really have here is a description of the octonions as a 'twisted group algebra'. Given any group $G$, the group algebra $\mathbb{R}[G]$ consists of all finite formal linear combinations of elements of $G$ with real coefficients. This is an associative algebra with the product coming from that of $G$. We can use any function

$$
\alpha: G^{2} \rightarrow\{ \pm 1\}
$$

to 'twist' this product, defining a new product

$$
\star: \mathbb{R}[G] \times \mathbb{R}[G] \rightarrow \mathbb{R}[G]
$$

by:

$$
g \star h=\alpha(g, h) g h,
$$

where $g, h \in G \subset \mathbb{R}[G]$. One can figure out an equation involving $\alpha$ that guarantees this new product will be associative. In this case we call $\alpha$ a '2-cocycle'. If $\alpha$ satisfies a certain extra equation, the product $\star$ will also be commutative, and we call $\alpha$ a 'stable 2-cocycle'. For example, the group algebra $\mathbb{R}\left[\mathbb{Z}_{2}\right]$ is isomorphic to a product of 2 copies of $\mathbb{R}$, but we can twist it by a stable 2-cocyle to obtain the complex numbers. The group algebra $\mathbb{R}\left[\mathbb{Z}_{2}^{2}\right]$ is isomorphic to a product of 4 copies of $\mathbb{R}$, but we can twist it by a 2-cocycle to obtain the quaternions. Similarly, the group algebra $\mathbb{R}\left[\mathbb{Z}_{2}^{3}\right]$ is a product of 8 copies of $\mathbb{R}$, and what we have really done in this section is describe a function $\alpha$ that allows us to twist this group algebra to obtain the octonions. Since the octonions are nonassociative, this function is not a 2cocycle. However, its coboundary is a 'stable 3-cocycle', which allows one to define a new associator and braiding for the category of $\mathbb{Z}_{2}^{3}$-graded vector spaces, making it into a symmetric monoidal category [4. In this symmetric monoidal category, the octonions are a commutative monoid object. In less technical terms: this category provides a context in which the octonions are commutative and associative! So far this idea has just begun to be exploited.

2.2. The Cayley-Dickson construction. It would be nice to have a construction of the normed division algebras $\mathbb{R}, \mathbb{C}, \mathbb{H}, \mathbb{O}$ that explained why each one fits neatly inside the next. It would be nice if this construction made it clear why $\mathbb{H}$ is noncommutative and $\mathbb{O}$ is nonassociative. It would be even better if this construction gave an infinite sequence of algebras, doubling in dimension each time, with the normed division algebras as the first four. In fact, there is such a construction: it's called the Cayley-Dickson construction.

As Hamilton noted, the complex number $a+b i$ can be thought of as a pair $(a, b)$ of real numbers. Addition is done component-wise, and multiplication goes like this:

$$
(a, b)(c, d)=(a c-d b, a d+c b) .
$$

We can also define the conjugate of a complex number by

$$
(a, b)^{*}=(a,-b) .
$$

Now that we have the complex numbers, we can define the quaternions in a similar way. A quaternion can be thought of as a pair of complex numbers. Addition 
is done component-wise, and multiplication goes like this:

$$
(a, b)(c, d)=\left(a c-d b^{*}, a^{*} d+c b\right) .
$$

This is just like our formula for multiplication of complex numbers, but with a couple of conjugates thrown in. If we included them in the previous formula, nothing would change, since the conjugate of a real number is just itself. We can also define the conjugate of a quaternion by

$$
(a, b)^{*}=\left(a^{*},-b\right) .
$$

The game continues! Now we can define an octonion to be a pair of quaternions. As before, we add and multiply them using formulas (2) and (3). This trick for getting new algebras from old is called the Cayley-Dickson construction.

Why do the real numbers, complex numbers, quaternions and octonions have multiplicative inverses? I take it as obvious for the real numbers. For the complex numbers, one can check that

$$
(a, b)(a, b)^{*}=(a, b)^{*}(a, b)=k(1,0)
$$

where $k$ is a real number, the square of the norm of $(a, b)$. This means that whenever $(a, b)$ is nonzero, its multiplicative inverse is $(a, b)^{*} / k$. One can check that the same holds for the quaternions and octonions.

But this, of course, raises the question: why isn't there an infinite sequence of division algebras, each one obtained from the preceding one by the Cayley-Dickson construction? The answer is that each time we apply the construction, our algebra gets a bit worse. First we lose the fact that every element is its own conjugate, then we lose commutativity, then we lose associativity, and finally we lose the division algebra property.

To see this clearly, it helps to be a bit more formal. Define a $*$-algebra to be an algebra $A$ equipped with a conjugation, that is, a real-linear map $*: A \rightarrow A$ with

$$
a^{* *}=a, \quad(a b)^{*}=b^{*} a^{*}
$$

for all $a, b \in A$. We say a $*$-algebra is real if $a=a^{*}$ for every element $a$ of the algebra. We say the $*$-algebra $A$ is nicely normed if $a+a^{*} \in \mathbb{R}$ and $a a^{*}=a^{*} a>0$ for all nonzero $a \in A$. If $A$ is nicely normed, we set

$$
\operatorname{Re}(a)=\left(a+a^{*}\right) / 2 \in \mathbb{R}, \quad \operatorname{Im}(a)=\left(a-a^{*}\right) / 2,
$$

and define a norm on $A$ by

$$
\|a\|^{2}=a a^{*} .
$$

If $A$ is nicely normed, it has multiplicative inverses given by

$$
a^{-1}=a^{*} /\|a\|^{2} .
$$

If $A$ is nicely normed and alternative, $A$ is a normed division algebra. To see this, note that for any $a, b \in A$, all 4 elements $a, b, a^{*}, b^{*}$ lie in the associative algebra generated by $\operatorname{Im}(a)$ and $\operatorname{Im}(b)$, so that

$$
\|a b\|^{2}=(a b)(a b)^{*}=a b\left(b^{*} a^{*}\right)=a\left(b b^{*}\right) a^{*}=\|a\|^{2}\|b\|^{2} .
$$

Starting from any *-algebra $A$, the Cayley-Dickson construction gives a new *algebra $A^{\prime}$. Elements of $A^{\prime}$ are pairs $(a, b) \in A^{2}$, and multiplication and conjugation are defined using equations (21) and (3). The following propositions show the effect of repeatedly applying the Cayley-Dickson construction:

Proposition 1. $A^{\prime}$ is never real. 
Proposition 2. $A$ is real (and thus commutative) $\Longleftrightarrow A^{\prime}$ is commutative.

Proposition 3. $A$ is commutative and associative $\Longleftrightarrow A^{\prime}$ is associative.

Proposition 4. $A$ is associative and nicely normed $\Longleftrightarrow A^{\prime}$ is alternative and nicely normed.

Proposition 5. $A$ is nicely normed $\Longleftrightarrow A^{\prime}$ is nicely normed.

All of these follow from straightforward calculations; to prove them here would merely deprive the reader of the pleasure of doing so. It follows from these propositions that:

$\mathbb{R}$ is a real commutative associative nicely normed $*$-algebra $\Longrightarrow$

$\mathbb{C}$ is a commutative associative nicely normed $*$-algebra $\Longrightarrow$

$\mathbb{H}$ is an associative nicely normed $*$-algebra $\Longrightarrow$

$\mathbb{O}$ is an alternative nicely normed $*$-algebra

and therefore that $\mathbb{R}, \mathbb{C}, \mathbb{H}$, and $\mathbb{O}$ are normed division algebras. It also follows that the octonions are neither real, nor commutative, nor associative.

If we keep applying the Cayley-Dickson process to the octonions, we get a sequence of $*$-algebras of dimension $16,32,64$, and so on. The first of these is called the sedenions, presumably alluding to the fact that it is 16 -dimensional 66. It follows from the above results that all the $*$-algebras in this sequence are nicely normed but neither real, nor commutative, nor alternative. They all have multiplicative inverses, since they are nicely normed. But they are not division algebras, since an explicit calculation demonstrates that the sedenions, and thus all the rest, have zero divisors. In fact [23], 72, the zero divisors of norm one in the sedenions form a subspace that is homeomorphic to the exceptional Lie group $\mathrm{G}_{2}$.

The Cayley-Dickson construction provides a nice way to obtain the sequence $\mathbb{R}, \mathbb{H}, \mathbb{C}, \mathbb{O}$ and the basic properties of these algebras. But what is the meaning of this construction? To answer this, it is better to define $A^{\prime}$ as the algebra formed by adjoining to $A$ an element $i$ satisfying $i^{2}=-1$ together with the following relations:

$$
a(i b)=i\left(a^{*} b\right), \quad(a i) b=\left(a b^{*}\right) i, \quad(i a)\left(b i^{-1}\right)=(a b)^{*}
$$

for all $a, b \in A$. We make $A^{\prime}$ into a $*$-algebra using the original conjugation on elements of $A$ and setting $i^{*}=-i$. It is easy to check that every element of $A^{\prime}$ can be uniquely written as $a+i b$ for some $a, b \in A$, and that this description of the Cayley-Dickson construction becomes equivalent to our previous one if we set $(a, b)=a+i b$.

What is the significance of the relations in (4)? Simply this: they express conjugation in terms of conjugation! This is a pun on the double meaning of the word 'conjugation'. What I really mean is that they express the $*$ operation in $A$ as conjugation by $i$. In particular, we have

$$
a^{*}=(i a) i^{-1}=i\left(a i^{-1}\right)
$$

for all $a \in A$. Note that when $A^{\prime}$ is associative, any one of the relations in (4) implies the other two. It is when $A^{\prime}$ is nonassociative that we really need all three relations.

This interpretation of the Cayley-Dickson construction makes it easier to see what happens as we repeatedly apply the construction starting with $\mathbb{R}$. In $\mathbb{R}$ the $*$ 
operation does nothing, so when we do the Cayley-Dickson construction, conjugation by $i$ must have no effect on elements of $\mathbb{R}$. Since $\mathbb{R}$ is commutative, this means that $\mathbb{C}=\mathbb{R}^{\prime}$ is commutative. But $\mathbb{C}$ is no longer real, since $i^{*}=-i$.

Next let us apply the Cayley-Dickson construction to $\mathbb{C}$. Since $\mathbb{C}$ is commutative, the $*$ operation in $\mathbb{C}$ is an automorphism. Whenever we have an associative algebra $A$ equipped with an automorphism $\alpha$, we can always extend $A$ to a larger associative algebra by adjoining an invertible element $x$ with

$$
\alpha(a)=x a x^{-1}
$$

for all $a \in A$. Since $\mathbb{C}$ is associative, this means that $\mathbb{C}^{\prime}=\mathbb{H}$ is associative. But since $\mathbb{C}$ is not real, $\mathbb{H}$ cannot be commutative, since conjugation by the newly adjoined element $i$ must have a nontrivial effect.

Finally, let us apply the Cayley-Dickson construction to $\mathbb{H}$. Since $\mathbb{H}$ is noncommutative, the $*$ operation in $\mathbb{H}$ is not an automorphism; it is merely an antiautomorphism. This means we cannot express it as conjugation by some element of a larger associative algebra. Thus $\mathbb{H}^{\prime}=\mathbb{O}$ must be nonassociative.

2.3. Clifford algebras. William Clifford invented his algebras in 1876 as an attempt to generalize the quaternions to higher dimensions, and he published a paper about them two years later 22 . Given a real inner product space $V$, the Clifford algebra $\operatorname{Cliff}(V)$ is the associative algebra freely generated by $V$ modulo the relations

$$
v^{2}=-\|v\|^{2}
$$

for all $v \in V$. Equivalently, it is the associative algebra freely generated by $V$ modulo the relations

$$
v w+w v=-2\langle v, w\rangle
$$

for all $v, w \in V$. If $V=\mathbb{R}^{n}$ with its usual inner product, we call this Clifford algebra $\operatorname{Cliff}(n)$. Concretely, this is the associative algebra freely generated by $n$ anticommuting square roots of -1 . From this we easily see that

$$
\operatorname{Cliff}(0)=\mathbb{R}, \quad \operatorname{Cliff}(1)=\mathbb{C}, \quad \operatorname{Cliff}(2)=\mathbb{H} .
$$

So far this sequence resembles the iterated Cayley-Dickson construction - but the octonions are not a Clifford algebra, since they are nonassociative. Nonetheless, there is a profound relation between Clifford algebras and normed division algebras. This relationship gives a nice way to prove that $\mathbb{R}, \mathbb{C}, \mathbb{H}$ and $\mathbb{O}$ are the only normed division algebras. It is also crucial for understanding the geometrical meaning of the octonions.

To see this relation, first suppose $\mathbb{K}$ is a normed division algebra. Left multiplication by any element $a \in \mathbb{K}$ gives an operator

$$
\begin{aligned}
& L_{a}: \mathbb{K} \rightarrow \mathbb{K} \\
& x \mapsto a x \text {. }
\end{aligned}
$$

If $\|a\|=1$, the operator $L_{a}$ is norm-preserving, so it maps the unit sphere of $\mathbb{K}$ to itself. Since $\mathbb{K}$ is a division algebra, we can find an operator of this form mapping any point on the unit sphere to any other point. The only way the unit sphere in $\mathbb{K}$ can have this much symmetry is if the norm on $\mathbb{K}$ comes from an inner product. Even better, this inner product is unique, since we can use the polarization identity

$$
\langle x, y\rangle=\frac{1}{2}\left(\|x+y\|^{2}-\|x\|^{2}-\|y\|^{2}\right)
$$


to recover it from the norm.

Using this inner product, we say an element $a \in \mathbb{K}$ is imaginary if it is orthogonal to the element 1 , and we let $\operatorname{Im}(\mathbb{K})$ be the space of imaginary elements of $\mathbb{K}$. We can also think of $\operatorname{Im}(\mathbb{K})$ as the tangent space of the unit sphere in $\mathbb{K}$ at the point 1. This has a nice consequence: since $a \mapsto L_{a}$ maps the unit sphere in $\mathbb{K}$ to the Lie group of orthogonal transformations of $\mathbb{K}$, it must send $\operatorname{Im}(\mathbb{K})$ to the Lie algebra of skew-adjoint transformations of $\mathbb{K}$. In short, $L_{a}$ is skew-adjoint whenever $a$ is imaginary.

The relation to Clifford algebras shows up when we compute the square of $L_{a}$ for $a \in \operatorname{Im}(\mathbb{K})$. We can do this most easily when $a$ has norm 1 . Then $L_{a}$ is both orthogonal and skew-adjoint. For any orthogonal transformation, we can find some orthonormal basis in which its matrix is block diagonal, built from $2 \times 2$ blocks that look like this:

$$
\left(\begin{array}{cc}
\cos \theta & \sin \theta \\
-\sin \theta & \cos \theta
\end{array}\right)
$$

and possibly a $1 \times 1$ block like this: (1). Such a transformation can only be skewadjoint if it consists solely of $2 \times 2$ blocks of this form:

$$
\pm\left(\begin{array}{cc}
0 & 1 \\
-1 & 0
\end{array}\right) \text {. }
$$

In this case, its square is -1 . We thus have $L_{a}^{2}=-1$ when $a \in \operatorname{Im}(\mathbb{K})$ has norm 1 . It follows that

$$
L_{a}^{2}=-\|a\|^{2}
$$

for all $a \in \operatorname{Im}(\mathbb{K})$. We thus obtain a representation of the Clifford algebra $\operatorname{Cliff}(\operatorname{Im}(\mathbb{K}))$ on $\mathbb{K}$. Any $n$-dimensional normed division algebra thus gives an $n$ dimensional representation of $\operatorname{Cliff}(n-1)$. As we shall see, this is very constraining.

We have already described the Clifford algebras up to Cliff(2). Further calculations [53], [77] give the following table, where we use $A[n]$ to stand for $n \times n$ matrices with entries in the algebra $A$ :

\begin{tabular}{|l|c|}
\hline$n$ & Cliff $(n)$ \\
\hline 0 & $\mathbb{R}$ \\
\hline 1 & $\mathbb{C}$ \\
\hline 2 & $\mathbb{H}$ \\
\hline 3 & $\mathbb{H} \oplus \mathbb{H}$ \\
\hline 4 & $\mathbb{H}[2]$ \\
\hline 5 & $\mathbb{C}[4]$ \\
\hline 6 & $\mathbb{R}[8]$ \\
\hline 7 & $\mathbb{R}[8] \oplus \mathbb{R}[8]$ \\
\hline
\end{tabular}

TABle 2. Clifford Algebras

Starting at dimension 8, something marvelous happens: the table continues in the following fashion:

$$
\operatorname{Cliff}(n+8) \cong \operatorname{Cliff}(n) \otimes \mathbb{R}[16]
$$


In other words, $\operatorname{Cliff}(n+8)$ consists of $16 \times 16$ matrices with entries in $\operatorname{Cliff}(n)$. This 'period-8' behavior was discovered by Cartan in 1908 [15], but we will take the liberty of calling it Bott periodicity, since it has a far-ranging set of applications to topology, some of which were discovered by Bott.

Since Clifford algebras are built from matrix algebras over $\mathbb{R}, \mathbb{C}$ and $\mathbb{H}$, it is easy to determine their representations. Every representation is a direct sum of irreducible ones. The only irreducible representation of $\mathbb{R}[n]$ is its obvious one via matrix multiplication on $\mathbb{R}^{n}$. Similarly, the only irreducible representation of $\mathbb{C}[n]$ is the obvious one on $\mathbb{C}^{n}$, and the only irreducible representation of $\mathbb{H}[n]$ is the obvious one on $\mathbb{H}^{n}$.

Glancing at the above table, we see that unless $n$ equals 3 or 7 modulo $8, \operatorname{Cliff}(n)$ is a real, complex or quaternionic matrix algebra, so it has a unique irreducible representation. For reasons to be explained later, this irreducible representation is known as the space of pinors and is denoted $P_{n}$. When $n$ is 3 or 7 modulo 8, the algebra $\operatorname{Cliff}(n)$ is a direct sum of two real or quaternionic matrix algebras, so it has two irreducible representations, which we call the positive pinors $P_{n}^{+}$and negative pinors $P_{n}^{-}$. We summarize these results in the following table:

\begin{tabular}{|l|c|l|}
\hline$n$ & Cliff $(n)$ & irreducible representations \\
\hline 0 & $\mathbb{R}$ & $P_{0}=\mathbb{R}$ \\
\hline 1 & $\mathbb{C}$ & $P_{1}=\mathbb{C}$ \\
\hline 2 & $\mathbb{H}$ & $P_{2}=\mathbb{H}$ \\
\hline 3 & $\mathbb{H} \oplus \mathbb{H}$ & $P_{3}^{+}=\mathbb{H}, P_{3}^{-}=\mathbb{H}$ \\
\hline 4 & $\mathbb{H}[2]$ & $P_{4}=\mathbb{H}{ }^{2}$ \\
\hline 5 & $\mathbb{C}[4]$ & $P_{5}=\mathbb{C}^{4}$ \\
\hline 6 & $\mathbb{R}[8]$ & $P_{6}=\mathbb{R}^{8}$ \\
\hline 7 & $\mathbb{R}[8] \oplus \mathbb{R}[8]$ & $P_{7}^{+}=\mathbb{R}^{8}, P_{7}^{-}=\mathbb{R}^{8}$ \\
\hline
\end{tabular}

TABle 3. Pinor Representations

Examining this table, we see that in the range of dimensions listed there is an $n$-dimensional representation of $\operatorname{Cliff}(n-1)$ only for $n=1,2,4$, and 8 . What about higher dimensions? By Bott periodicity, the irreducible representations of Cliff $(n+8)$ are obtained by tensoring those of $\operatorname{Cliff}(n)$ by $\mathbb{R}^{16}$. This multiplies the dimension by 16 , so one can easily check that for $n>8$, the irreducible representations of $\operatorname{Cliff}(n-1)$ always have dimension greater than $n$.

It follows that normed division algebras are only possible in dimensions $1,2,4$, and 8. Having constructed $\mathbb{R}, \mathbb{C}, \mathbb{H}$ and $\mathbb{O}$, we also know that normed division algebras exist in these dimensions. The only remaining question is whether they are unique. For this it helps to investigate more deeply the relation between normed division algebras and the Cayley-Dickson construction. In what follows, we outline an approach based on ideas in the book by Springer and Veldkamp [89].

First, suppose $\mathbb{K}$ is a normed division algebra. Then there is a unique linear operator $*: \mathbb{K} \rightarrow \mathbb{K}$ such that $1^{*}=1$ and $a^{*}=-a$ for $a \in \operatorname{Im}(\mathbb{K})$. With some calculation one can prove this makes $\mathbb{K}$ into a nicely normed $*$-algebra.

Next, suppose that $\mathbb{K}_{0}$ is any subalgebra of the normed division algebra $\mathbb{K}$. It is easy to check that $\mathbb{K}_{0}$ is a nicely normed $*$-algebra in its own right. If $\mathbb{K}_{0}$ is 
not all of $\mathbb{K}$, we can find an element $i \in \mathbb{K}$ that is orthogonal to every element of $\mathbb{K}_{0}$. Without loss of generality we shall assume this element has norm 1 . Since this element $i$ is orthogonal to $1 \in \mathbb{K}_{0}$, it is imaginary. From the definition of the * operator it follows that $i^{*}=-i$, and from results earlier in this section we have $i^{2}=-1$. With further calculation one can show that for all $a, a^{\prime} \in \mathbb{K}_{0}$ we have

$$
a\left(i a^{\prime}\right)=i\left(a^{*} a^{\prime}\right), \quad(a i) a^{\prime}=\left(a a^{*}\right) i, \quad(i a)\left(a^{\prime} i^{-1}\right)=\left(a a^{\prime}\right)^{*} .
$$

A glance at equation (4) reveals that these are exactly the relations defining the Cayley-Dickson construction! With a little thought, it follows that the subalgebra of $\mathbb{K}$ generated by $\mathbb{K}_{0}$ and $i$ is isomorphic as a $*$-algebra to $\mathbb{K}_{0}^{\prime}$, the $*$-algebra obtained from $\mathbb{K}_{0}$ by the Cayley-Dickson construction.

Thus, whenever we have a normed division algebra $\mathbb{K}$ we can find a chain of subalgebras $\mathbb{R}=\mathbb{K}_{0} \subset \mathbb{K}_{1} \subset \cdots \subset \mathbb{K}_{n}=\mathbb{K}$ such that $\mathbb{K}_{i+1} \cong \mathbb{K}_{i}^{\prime}$. To construct $\mathbb{K}_{i+1}$, we simply need to choose a norm-one element of $\mathbb{K}$ that is orthogonal to every element of $\mathbb{K}_{i}$. It follows that the only normed division algebras of dimension $1,2,4$ and 8 are $\mathbb{R}, \mathbb{C}, \mathbb{H}$ and $\mathbb{O}$. This also gives an alternate proof that there are no normed division algebras of other dimensions: if there were any, there would have to be a 16-dimensional one, namely $\mathbb{O}^{\prime}$ - the sedenions. But as mentioned in Section [2.2, one can check explicitly that the sedenions are not a division algebra.

2.4. Spinors and trialities. A nonassociative division algebra may seem like a strange thing to bother with, but the notion of triality makes it seem a bit more natural. The concept of duality is important throughout linear algebra. The concept of triality is similar, but considerably subtler. Given vector spaces $V_{1}$ and $V_{2}$, we may define a duality to be a bilinear map

$$
f: V_{1} \times V_{2} \rightarrow \mathbb{R}
$$

that is nondegenerate, meaning that if we fix either argument to any nonzero value, the linear functional induced on the other vector space is nonzero. Similarly, given vector spaces $V_{1}, V_{2}$, and $V_{3}$, a triality is a trilinear map

$$
t: V_{1} \times V_{2} \times V_{3} \rightarrow \mathbb{R}
$$

that is nondegenerate in the sense that if we fix any two arguments to any nonzero values, the linear functional induced on the third vector space is nonzero.

Dualities are easy to come by. Trialities are much rarer, for suppose we have a triality

$$
t: V_{1} \times V_{2} \times V_{3} \rightarrow \mathbb{R}
$$

By dualizing, we can turn this into a bilinear map

$$
m: V_{1} \times V_{2} \rightarrow V_{3}^{*}
$$

which we call 'multiplication'. By the nondegeneracy of our triality, left multiplication by any nonzero element of $V_{1}$ defines an isomorphism from $V_{2}$ to $V_{3}^{*}$. Similarly, right multiplication by any nonzero element of $V_{2}$ defines an isomorphism from $V_{1}$ to $V_{3}^{*}$. If we choose nonzero elements $e_{1} \in V_{1}$ and $e_{2} \in V_{2}$, we can thereby identify the spaces $V_{1}, V_{2}$ and $V_{3}^{*}$ with a single vector space, say $V$. Note that this identifies all three vectors $e_{1} \in V_{1}, e_{2} \in V_{2}$, and $e_{1} e_{2} \in V_{3}^{*}$ with the same vector $e \in V$. We thus obtain a product

$$
m: V \times V \rightarrow V
$$


for which $e$ is the left and right unit. Since left or right multiplication by any nonzero element is an isomorphism, $V$ is actually a division algebra! Conversely, any division algebra gives a triality.

It follows from Theorem 3 that trialities only occur in dimensions 1, 2, 4, or 8. This theorem is quite deep. By comparison, Hurwitz's classification of normed division algebras is easy to prove. Not surprisingly, these correspond to a special sort of triality, which we call a 'normed' triality.

To be precise, a normed triality consists of inner product spaces $V_{1}, V_{2}, V_{3}$ equipped with a trilinear map $t: V_{1} \times V_{2} \times V_{3} \rightarrow \mathbb{R}$ with

$$
\left|t\left(v_{1}, v_{2}, v_{3}\right)\right| \leq\left\|v_{1}\right\|\left\|v_{2}\right\|\left\|v_{3}\right\|
$$

and such that for all $v_{1}, v_{2}$ there exists $v_{3} \neq 0$ for which this bound is attained and similarly for cyclic permutations of $1,2,3$. Given a normed triality, picking unit vectors in any two of the spaces $V_{i}$ allows us to identify all three spaces and get a normed division algebra. Conversely, any normed division algebra gives a normed triality.

But where do normed trialities come from? They come from the theory of spinors! From Section 2.3. we already know that any $n$-dimensional normed division algebra is a representation of $\operatorname{Cliff}(n-1)$, so it makes sense to look for normed trialities here. In fact, representations of $\operatorname{Cliff}(n-1)$ give certain representations of $\operatorname{Spin}(n)$, the double cover of the rotation group in $n$ dimensions. These are called 'spinors'. As we shall see, the relation between spinors and vectors gives a nice way to construct normed trialities in dimensions $1,2,4$ and 8.

To see how this works, first let $\operatorname{Pin}(n)$ be the group sitting inside $\operatorname{Cliff}(n)$ that consists of all products of unit vectors in $\mathbb{R}^{n}$. This group is a double cover of the orthogonal group $\mathrm{O}(n)$, where given any unit vector $v \in \mathbb{R}^{n}$, we map both $\pm v \in$ $\operatorname{Pin}(n)$ to the element of $\mathrm{O}(n)$ that reflects across the hyperplane perpendicular to $v$. Since every element of $\mathrm{O}(n)$ is a product of reflections, this homomorphism is indeed onto.

Next, let $\operatorname{Spin}(n) \subset \operatorname{Pin}(n)$ be the subgroup consisting of all elements that are a product of an even number of unit vectors in $\mathbb{R}^{n}$. An element of $\mathrm{O}(n)$ has determinant 1 iff it is the product of an even number of reflections, so just as $\operatorname{Pin}(n)$ is a double cover of $\mathrm{O}(n), \operatorname{Spin}(n)$ is a double cover of $\mathrm{SO}(n)$. Together with a French dirty joke which we shall not explain, this analogy is the origin of the terms 'Pin' and 'pinor'.

Since $\operatorname{Pin}(n)$ sits inside $\operatorname{Cliff}(n)$, the irreducible representations of Cliff $(n)$ restrict to representations of $\operatorname{Pin}(n)$, which turn out to be still irreducible. These are again called pinors, and we know what they are from Table 3. Similarly, $\operatorname{Spin}(n)$ sits inside the subalgebra

$$
\operatorname{Cliff}_{0}(n) \subseteq \operatorname{Cliff}(n)
$$

consisting of all linear combinations of products of an even number of vectors in $\mathbb{R}^{n}$. Thus the irreducible representations of $\operatorname{Cliff}_{0}(n)$ restrict to representations of $\operatorname{Spin}(n)$, which turn out to be still irreducible. These are called spinors - but we warn the reader that this term is also used for many slight variations on this concept.

In fact, there is an isomorphism

$$
\phi: \operatorname{Cliff}(n-1) \rightarrow \operatorname{Cliff}_{0}(n)
$$


given as follows:

$$
\phi\left(e_{i}\right)=e_{i} e_{n}, \quad 1 \leq i \leq n-1,
$$

where $\left\{e_{i}\right\}$ is an orthonormal basis for $\mathbb{R}^{n}$. Thus spinors in $n$ dimensions are the same as pinors in $n-1$ dimensions! Table 3 therefore yields the following table, where we use similar notation but with ' $S$ ' instead of ' $P$ ':

\begin{tabular}{|l|c|l|}
\hline$n$ & Cliff $_{0}(n)$ & irreducible representations \\
\hline 1 & $\mathbb{R}$ & $S_{1}=\mathbb{R}$ \\
\hline 2 & $\mathbb{C}$ & $S_{2}=\mathbb{C}$ \\
\hline 3 & $\mathbb{H}$ & $S_{3}=\mathbb{H}$ \\
\hline 4 & $\mathbb{H} \oplus \mathbb{H}$ & $S_{4}^{+}=\mathbb{H}, S_{4}^{-}=\mathbb{H}$ \\
\hline 5 & $\mathbb{H}[2]$ & $S_{5}=\mathbb{H}^{2}$ \\
\hline 6 & $\mathbb{C}[4]$ & $S_{6}=\mathbb{C}^{4}$ \\
\hline 7 & $\mathbb{R}[8]$ & $S_{7}=\mathbb{R}^{8}$ \\
\hline 8 & $\mathbb{R}[8] \oplus \mathbb{R}[8]$ & $S_{8}^{+}=\mathbb{R}^{8}, S_{8}^{-}=\mathbb{R}^{8}$ \\
\hline
\end{tabular}

TABLE 4. Spinor Representations

We call $S_{n}^{+}$and $S_{n}^{-}$the right-handed and left-handed spinor representations. For $n>8$ we can work out the spinor representations using Bott periodicity:

$$
S_{n+8} \cong S_{n} \otimes \mathbb{R}^{16}
$$

and similarly for right-handed and left-handed spinors.

Now, besides its pinor representation(s), the group $\operatorname{Pin}(n)$ also has an irreducible representation where we first apply the 2-1 homomorphism $\operatorname{Pin}(n) \rightarrow \mathrm{O}(n)$ and then use the obvious representation of $\mathrm{O}(n)$ on $\mathbb{R}^{n}$. We call this the vector representation, $V_{n}$. As a vector space $V_{n}$ is just $\mathbb{R}^{n}$, and $\operatorname{Cliff}(n)$ is generated by $\mathbb{R}^{n}$, so we have an inclusion

$$
V_{n} \hookrightarrow \operatorname{Cliff}(n)
$$

Using this, we can restrict the action of the Clifford algebra on pinors to a map

$$
\begin{array}{lc}
m_{n}: & V_{n} \times P_{n}^{ \pm} \rightarrow P_{n}^{ \pm} \quad n \equiv 3,7 \bmod 8 \\
m_{n}: & V_{n} \times P_{n} \rightarrow P_{n} \quad \text { otherwise. }
\end{array}
$$

This map is actually an intertwining operator between representations of $\operatorname{Pin}(n)$. If we restrict the vector representation to the subgroup $\operatorname{Spin}(n)$, it remains irreducible. This is not always true for the pinor representations, but we can always decompose them as a direct sum of spinor representations. Applying this decomposition to the map $m_{n}$, we get a map

$$
\begin{array}{lc}
m_{n}: V_{n} \times S_{n}^{ \pm} \rightarrow S_{n}^{\mp} & n \equiv 0,4 \bmod 8 \\
m_{n}: V_{n} \times S_{n} \rightarrow S_{n} & \text { otherwise. }
\end{array}
$$

All the spinor representations appearing here are self-dual, so we can dualize the above maps and reinterpret them as trilinear maps:

$$
\begin{array}{lc}
t_{n}: V_{n} \times S_{n}^{+} \times S_{n}^{-} \rightarrow \mathbb{R} & n \equiv 0,4 \bmod 8 \\
t_{n}: V_{n} \times S_{n} \times S_{n} \rightarrow \mathbb{R} \quad \text { otherwise. }
\end{array}
$$


These trilinear maps are candidates for trialities! However, they can only be trialities when the dimension of the vector representation matches that of the relevant spinor representations. In the range of the above table this happens only for $n=1,2,4,8$. In these cases we actually do get normed trialities, which in turn give normed division algebras:

$$
\begin{array}{lll}
t_{1}: V_{1} \times S_{1} \times S_{1} \rightarrow \mathbb{R} & \text { gives } \mathbb{R} . \\
t_{2}: V_{2} \times S_{2} \times S_{2} \rightarrow \mathbb{R} & \text { gives } \mathbb{C} . \\
t_{4}: V_{4} \times S_{4}^{+} \times S_{4}^{-} \rightarrow \mathbb{R} & \text { gives } \mathbb{H} . \\
t_{8}: V_{8} \times S_{8}^{+} \times S_{8}^{-} \rightarrow \mathbb{R} & \text { gives } \mathbb{O} .
\end{array}
$$

In higher dimensions, the spinor representations become bigger than the vector representation, so we get no more trialities this way - and of course, none exist.

Of the four normed trialities, the one that gives the octonions has an interesting property that the rest lack. To see this property, one must pay careful attention to the difference between a normed triality and a normed division algebra. To construct a normed division $\mathbb{K}$ algebra from the normed triality $t: V_{1} \times V_{2} \times V_{3} \rightarrow \mathbb{R}$, we must arbitrarily choose unit vectors in two of the three spaces, so the symmetry group of $\mathbb{K}$ is smaller than that of $t$. More precisely, let us define an automorphism of the normed triality $t: V_{1} \times V_{2} \times V_{3} \rightarrow \mathbb{R}$ to be a triple of norm-preserving maps $f_{i}: V_{i} \rightarrow V_{i}$ such that

$$
t\left(f_{1}\left(v_{1}\right), f_{2}\left(v_{2}\right), f_{3}\left(v_{3}\right)\right)=t\left(v_{1}, v_{2}, v_{3}\right)
$$

for all $v_{i} \in V_{i}$. These automorphisms form a group we call $\operatorname{Aut}(t)$. If we construct a normed division algebra $\mathbb{K}$ from $t$ by choosing unit vectors $e_{1} \in V_{1}, e_{2} \in V_{2}$, we have

$$
\operatorname{Aut}(\mathbb{K}) \cong\left\{\left(f_{1}, f_{2}, f_{3}\right) \in \operatorname{Aut}(t): f_{1}\left(e_{1}\right)=e_{1}, f_{2}\left(e_{2}\right)=e_{2}\right\} .
$$

In particular, it turns out that:

$$
\begin{aligned}
& 1 \cong \operatorname{Aut}(\mathbb{R}) \subseteq \operatorname{Aut}\left(t_{1}\right) \cong\left\{\left(g_{1}, g_{2}, g_{3}\right) \in \mathrm{O}(1)^{3}: g_{1} g_{2} g_{3}=1\right\} \\
& \mathbb{Z}_{2} \cong \operatorname{Aut}(\mathbb{C}) \subseteq \operatorname{Aut}\left(t_{2}\right) \cong\left\{\left(g_{1}, g_{2}, g_{3}\right) \in \mathrm{U}(1)^{3}: g_{1} g_{2} g_{3}=1\right\} \times \mathbb{Z}_{2} \\
& \mathrm{SO}(3) \cong \operatorname{Aut}(\mathbb{H}) \subseteq \operatorname{Aut}\left(t_{4}\right) \cong \operatorname{Sp}(1)^{3} /\{ \pm(1,1,1)\} \\
& \mathrm{G}_{2} \cong \operatorname{Aut}(\mathbb{O}) \subseteq \operatorname{Aut}\left(t_{8}\right) \cong \operatorname{Spin}(8)
\end{aligned}
$$

where

$$
\mathrm{O}(1) \cong \mathbb{Z}_{2}, \quad \mathrm{U}(1) \cong \mathrm{SO}(2), \quad \mathrm{Sp}(1) \cong \mathrm{SU}(2)
$$

are the unit spheres in $\mathbb{R}, \mathbb{C}$ and $\mathbb{H}$, respectively — the only spheres that are Lie groups. $\mathrm{G}_{2}$ is just another name for the automorphism group of the octonions; we shall study this group in Section 4.1. The bigger group Spin(8) acts as automorphisms of the triality that gives the octonions, and it does so in an interesting way. Given any element $g \in \operatorname{Spin}(8)$, there exist unique elements $g_{ \pm} \in \operatorname{Spin}(8)$ such that

$$
t\left(g\left(v_{1}\right), g_{+}\left(v_{2}\right), g_{-}\left(v_{3}\right)\right)=t\left(v_{1}, v_{2}, v_{3}\right)
$$

for all $v_{1} \in V_{8}, v_{2} \in S_{8}^{+}$, and $v_{3} \in S_{8}^{-}$. Moreover, the maps

$$
\alpha_{ \pm}: g \rightarrow g_{ \pm}
$$

are outer automorphisms of $\operatorname{Spin}(8)$. In fact Out(Spin(8)) is the permutation group on 3 letters, and there exist outer automorphisms that have the effect of permuting the vector, left-handed spinor, and right-handed spinor representations any way one likes; $\alpha_{+}$and $\alpha_{-}$are among these. 
In general, outer automorphisms of simple Lie groups come from symmetries of their Dynkin diagrams. Of all the simple Lie groups, Spin(8) has the most symmetrical Dynkin diagram! It looks like this:

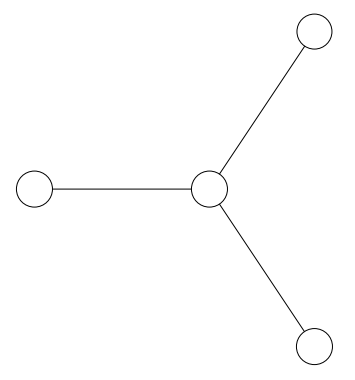

Here the three outer nodes correspond to the vector, left-handed spinor and righthanded spinor representations of $\operatorname{Spin}(8)$, while the central node corresponds to the adjoint representation - that is, the representation of $\operatorname{Spin}(8)$ on its own Lie algebra, better known as $\mathfrak{s o}(8)$. The outer automorphisms corresponding to the symmetries of this diagram were discovered in 1925 by Cartan [16, who called these symmetries triality. The more general notion of 'triality' we have been discussing here came later and is apparently due to Adams [2].

The construction of division algebras from trialities has tantalizing links to physics. In the Standard Model of particle physics, all particles other than the Higgs boson transform either as vectors or spinors. The vector particles are also called 'gauge bosons', and they serve to carry the forces in the Standard Model. The spinor particles are also called 'fermions', and they correspond to the basic forms of matter: quarks and leptons. The interaction between matter and the forces is described by a trilinear map involving two spinors and one vector. This map is often drawn as a Feynman diagram:

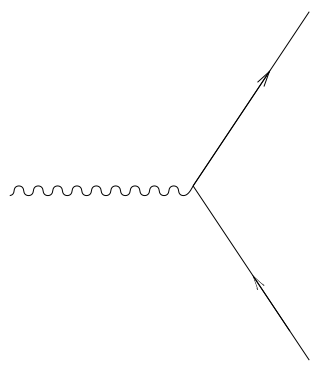

where the straight lines denote spinors and the wiggly one denotes a vector. The most familiar example is the process whereby an electron emits or absorbs a photon.

It is fascinating that the same sort of mathematics can be used both to construct the normed division algebras and to describe the interaction between matter and forces. Could this be important for physics? One prima facie problem with this speculation is that physics uses spinors associated to Lorentz groups rather 
than rotation groups, due to the fact that spacetime has a Lorentzian rather than Euclidean metric. However, in Section [3.3] we describe a way around this problem. Just as octonions give the spinor representations of $\operatorname{Spin}(8)$, pairs of octonions give the spinor representations of $\operatorname{Spin}(9,1)$. This is one reason so many theories of physics work best when spacetime is 10-dimensional! Examples include superstring theory [28], 45], supersymmetric gauge theories [34, [63], 84, and Geoffrey Dixon's extension of the Standard Model based on the algebra $\mathbb{C} \otimes \mathbb{H} \otimes \mathbb{O}$, in which the 3 forces arise naturally from the three factors in this tensor product 30 .

\section{OCTONIONIC PROJECTIVE GEOMETRY}

Projective geometry is a venerable subject that has its origins in the study of perspective by Renaissance painters. As seen by the eye, parallel lines - e.g., train tracks - appear to meet at a 'point at infinity'. When one changes ones viewpoint, distances and angles appear to change, but points remain points and lines remain lines. These facts suggest a modification of Euclidean plane geometry, based on a set of points, a set of lines, and relation whereby a point 'lies on' a line, satisfying the following axioms:

- For any two distinct points, there is a unique line on which they both lie.

- For any two distinct lines, there is a unique point which lies on both of them.

- There exist four points, no three of which lie on the same line.

- There exist four lines, no three of which have the same point lying on them.

A structure satisfying these axioms is called a projective plane. Part of the charm of this definition is that it is 'self-dual': if we switch the words 'point' and 'line' and switch who lies on whom, it stays the same.

We have already met one example of a projective plane in Section 2.1; the smallest one of all, the Fano plane. The example relevant to perspective is the real projective plane, $\mathbb{R P}^{2}$. Here the points are lines through the origin in $\mathbb{R}^{3}$, the lines are planes through the origin in $\mathbb{R}^{3}$, and the relation of 'lying on' is taken to be inclusion. Each point $(x, y) \in \mathbb{R}^{2}$ determines a point in $\mathbb{R P}^{2}$, namely the line in $\mathbb{R}^{3}$ containing the origin and the point $(x, y,-1)$ :

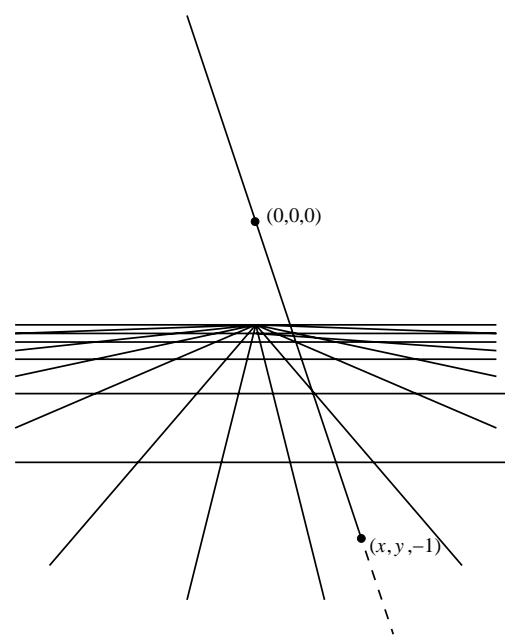


There are also other points in $\mathbb{R P}^{2}$, the 'points at infinity', corresponding to lines through the origin in $\mathbb{R}^{3}$ that do not intersect the plane $\{z=-1\}$. For example, any point on the horizon in the above picture determines a point at infinity.

Projective geometry is also interesting in higher dimensions. One can define a projective space by the following axioms:

- For any two distinct points $p, q$, there is a unique line $p q$ on which they both lie.

- For any line, there are at least three points lying on this line.

- If $a, b, c, d$ are distinct points and there is a point lying on both $a b$ and $c d$, then there is a point lying on both $a c$ and $b d$.

Given a projective space and a set $S$ of points in this space, we define the span of $S$ to be the smallest set $T$ of points containing $S$ such that if $a$ and $b$ lie in $T$, so do all points on the line $a b$. The dimension of a projective space is defined to be one less than the minimal cardinality of a set that spans the whole space. The reader may enjoy showing that a 2 -dimensional projective space is the same thing as a projective plane [43].

If $\mathbb{K}$ is any field, there is an $n$-dimensional projective space called $\mathbb{K} \mathbb{P}^{n}$ where the points are lines through the origin in $\mathbb{K}^{n+1}$, the lines are planes through the origin in $\mathbb{K}^{n+1}$, and the relation of 'lying on' is inclusion. In fact, this construction works even when $\mathbb{K}$ is a mere skew field: a ring such that every nonzero element has a left and right multiplicative inverse. We just need to be a bit careful about defining lines and planes through the origin in $\mathbb{K}^{n+1}$. To do this, we use the fact that $\mathbb{K}^{n+1}$ is a $\mathbb{K}$-bimodule in an obvious way. We take a line through the origin to be any set

$$
L=\{\alpha x: \alpha \in \mathbb{K}\}
$$

where $x \in \mathbb{K}^{n+1}$ is nonzero, and take a plane through the origin to be any set

$$
P=\{\alpha x+\beta y: \alpha, \beta \in \mathbb{K}\}
$$

where $x, y \in \mathbb{K}^{n+1}$ are elements such that $\alpha x+\beta y=0$ implies $\alpha, \beta=0$.

Given this example, the question naturally arises whether every projective $n$ space is of the form $\mathbb{K} \mathbb{P}^{n}$ for some skew field $\mathbb{K}$. The answer is quite surprising: yes, but only if $n>2$. Projective planes are more subtle [90]. A projective plane comes from a skew field if and only if it satisfies an extra axiom, the 'axiom of Desargues', which goes as follows. Define a triangle to be a triple of points that don't all lie on the same line. Now, suppose we have two triangles $x y z$ and $x^{\prime} y^{\prime} z^{\prime}$. The sides of each triangle determine three lines, say $L M N$ and $L^{\prime} M^{\prime} N^{\prime}$. Sometimes the line through $x$ and $x^{\prime}$, the line through $y$ and $y^{\prime}$, and the line through $z$ and $z^{\prime}$ will all intersect at the same point: 


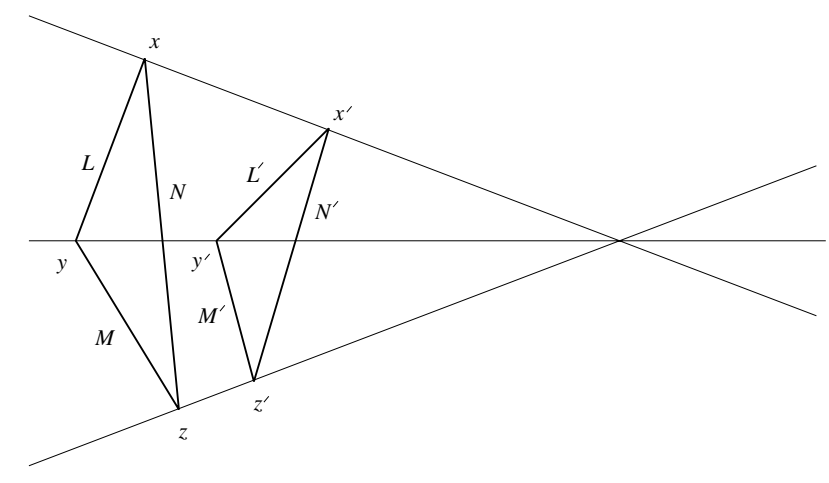

The axiom of Desargues says that whenever this happens, something else happens: the intersection of $L$ and $L^{\prime}$, the intersection of $M$ and $M^{\prime}$, and the intersection of $N$ and $N^{\prime}$ all lie on the same line:

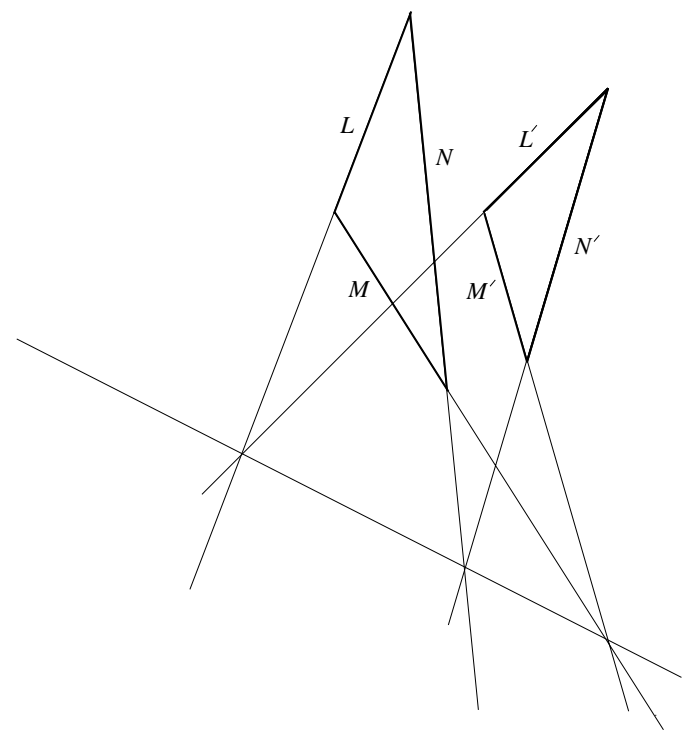

This axiom holds automatically for projective spaces of dimension 3 or more, but not for projective planes. A projective plane satisfying this axiom is called Desarguesian.

The axiom of Desargues is pretty, but what is its connection to skew fields? Suppose we start with a projective plane $P$ and try to reconstruct a skew field from it. We can choose any line $L$, choose three distinct points on this line, call them 0,1 , and $\infty$, and set $\mathbb{K}=L-\{\infty\}$. Copying geometric constructions that work when $P=\mathbb{R P}^{2}$, we can define addition and multiplication of points in $\mathbb{K}$. In general the resulting structure $(\mathbb{K},+, 0, \cdot, 1)$ will not be a skew field. Even worse, it will depend in a nontrivial way on the choices made. However, if we assume the axiom 
of Desargues, these problems go away. We thus obtain a one-to-one correspondence between isomorphism classes of skew fields and isomorphism classes of Desarguesian projective planes.

Projective geometry was very fashionable in the 1800 s, with such worthies as Poncelet, Brianchon, Steiner and von Staudt making important contributions. Later it was overshadowed by other forms of geometry. However, work on the subject continued, and in 1933 Ruth Moufang constructed a remarkable example of a nonDesarguesian projective plane using the octonions 73. As we shall see, this projective plane deserves the name $\mathbb{O P}^{2}$.

The 1930s also saw the rise of another reason for interest in projective geometry: quantum mechanics! Quantum theory is distressingly different from the classical Newtonian physics we have learnt to love. In classical mechanics, observables are described by real-valued functions. In quantum mechanics, they are often described by hermitian $n \times n$ complex matrices. In both cases, observables are closed under addition and multiplication by real scalars. However, in quantum mechanics, observables do not form an associative algebra. Still, one can raise an observable to a power, and from squaring one can construct a commutative but nonassociative product:

$$
a \circ b=\frac{1}{2}\left((a+b)^{2}-a^{2}-b^{2}\right)=\frac{1}{2}(a b+b a) .
$$

In 1932, Pascual Jordan attempted to understand this situation better by isolating the bare minimum axioms that an 'algebra of observables' should satisfy [56. He invented the definition of what is now called a formally real Jordan algebra: a commutative and power-associative algebra satisfying

$$
a_{1}^{2}+\cdots+a_{n}^{2}=0 \quad \Longrightarrow \quad a_{1}=\cdots=a_{n}=0
$$

for all $n$. The last condition gives the algebra a partial ordering: if we write $a \leq b$ when the element $b-a$ is a sum of squares, it says that $a \leq b$ and $b \leq a$ imply $a=b$. Though it is not obvious, any formally real Jordan algebra satisfies the identity

$$
a \circ\left(b \circ a^{2}\right)=(a \circ b) \circ a^{2}
$$

for all elements $a$ and $b$. Any commutative algebra satisfying this identity is called a Jordan algebra. Jordan algebras are automatically power-associative.

In 1934, Jordan published a paper with von Neumann and Wigner classifying all formally real Jordan algebras [58]. The classification is nice and succinct. An ideal in the Jordan algebra $A$ is a subspace $B \subseteq A$ such that $b \in B$ implies $a \circ b \in B$ for all $a \in A$. A Jordan algebra $A$ is simple if its only ideals are $\{0\}$ and $A$ itself. Every formally real Jordan algebra is a direct sum of simple ones. The simple formally real Jordan algebras consist of 4 infinite families and one exception.

1. The algebra $\mathfrak{h}_{n}(\mathbb{R})$ with the product $a \circ b=\frac{1}{2}(a b+b a)$.

2. The algebra $\mathfrak{h}_{n}(\mathbb{C})$ with the product $a \circ b=\frac{1}{2}(a b+b a)$.

3. The algebra $\mathfrak{h}_{n}(\mathbb{H})$ with the product $a \circ b=\frac{1}{2}(a b+b a)$.

4. The algebra $\mathbb{R}^{n} \oplus \mathbb{R}$ with the product

$$
(v, \alpha) \circ(w, \beta)=(\alpha w+\beta v,\langle v, w\rangle+\alpha \beta) .
$$

5. The algebra $\mathfrak{h}_{3}(\mathbb{O})$ with the product $a \circ b=\frac{1}{2}(a b+b a)$. 
Here we say a square matrix with entries in the $*$-algebra $A$ is hermitian if it equals its conjugate transpose, and we let $\mathfrak{h}_{n}(A)$ stand for the hermitian $n \times n$ matrices with entries in $A$. Jordan algebras in the fourth family are called spin factors, while $\mathfrak{h}_{3}(\mathbb{O})$ is called the exceptional Jordan algebra. This classification raises some obvious questions. Why does nature prefer the Jordan algebras $\mathfrak{h}_{n}(\mathbb{C})$ over all the rest? Or does it? Could the other Jordan algebras - even the exceptional one - have some role to play in quantum physics? Despite much research, these questions remain unanswered to this day.

The paper by Jordan, von Neumann and Wigner appears to have been uninfluenced by Moufang's discovery of $\mathbb{O P}^{2}$, but in fact they are related. A projection in a formally real Jordan algebra is defined to be an element $p$ with $p^{2}=p$. In the familiar case of $\mathfrak{h}_{n}(\mathbb{C})$, these correspond to hermitian matrices with eigenvalues 0 and 1 , so they are used to describe observables that assume only two values e.g., 'true' and 'false'. This suggests treating projections in a formally real Jordan algebra as propositions in a kind of 'quantum logic'. The partial order helps us do this: given projections $p$ and $q$, we say that $p$ 'implies' $q$ if $p \leq q$.

The relation between Jordan algebras and quantum logic is already interesting 33. but the real fun starts when we note that projections in $\mathfrak{h}_{n}(\mathbb{C})$ correspond to subspaces of $\mathbb{C}^{n}$. This sets up a relationship to projective geometry [97, since the projections onto 1-dimensional subspaces correspond to points in $\mathbb{C P}^{n}$, while the projections onto 2-dimensional subspaces correspond to lines. Even better, we can work out the dimension of a subspace $V \subseteq \mathbb{C}^{n}$ from the corresponding projection $p: \mathbb{C}^{n} \rightarrow V$ using only the partial order on projections: $V$ has dimension $d$ iff the longest chain of distinct projections

$$
0=p_{0}<\cdots<p_{i}=p
$$

has length $i=d$. In fact, we can use this to define the rank of a projection in any formally real Jordan algebra. We can then try to construct a projective space whose points are the rank-1 projections and whose lines are the rank-2 projections, with the relation of 'lying on' given by the partial order $\leq$.

If we try this starting with $\mathfrak{h}_{n}(\mathbb{R}), \mathfrak{h}_{n}(\mathbb{C})$ or $\mathfrak{h}_{n}(\mathbb{H})$, we succeed when $n \geq 2$, and we obtain the projective spaces $\mathbb{R P}^{n}, \mathbb{C P}^{n}$ and $\mathbb{H P}^{n}$, respectively. If we try this starting with the spin factor $\mathbb{R}^{n} \oplus \mathbb{R}$, we succeed when $n \geq 2$ and obtain a series of 1-dimensional projective spaces related to Lorentzian geometry. Finally, in 1949 Jordan [57] discovered that if we try this construction starting with the exceptional Jordan algebra, we get the projective plane discovered by Moufang: $\mathbb{O P}^{2}$.

In what follows we describe the octonionic projective plane and exceptional Jordan algebra in more detail. But first let us consider the octonionic projective line and the Jordan algebra $\mathfrak{h}_{2}(\mathbb{O})$.

3.1. Projective lines. A one-dimensional projective space is called a projective line. Projective lines are not very interesting from the viewpoint of axiomatic projective geometry, since they have only one line on which all the points lie. Nonetheless, they can be geometrically and topologically interesting. This is especially true of the octonionic projective line. As we shall see, this space has a deep connection to Bott periodicity, and also to the Lorentzian geometry of 10-dimensional spacetime.

Suppose $\mathbb{K}$ is a normed division algebra. We have already defined $\mathbb{K} \mathbb{P}^{1}$ when $\mathbb{K}$ is associative, but this definition does not work well for the octonions: it is wiser to take a detour through Jordan algebras. Let $\mathfrak{h}_{2}(\mathbb{K})$ be the space of $2 \times 2$ hermitian 
matrices with entries in $\mathbb{K}$. It is easy to check that this becomes a Jordan algebra with the product $a \circ b=\frac{1}{2}(a b+b a)$. We can try to build a projective space from this Jordan algebra using the construction in the previous section. To see if this succeeds, we need to ponder the projections in $\mathfrak{h}_{2}(\mathbb{K})$. A little calculation shows that besides the trivial projections 0 and 1 , they are all of the form

$$
\left(\begin{array}{l}
x^{*} \\
y^{*}
\end{array}\right)\left(\begin{array}{ll}
x & y
\end{array}\right)=\left(\begin{array}{cc}
x^{*} x & x^{*} y \\
y^{*} x & y^{*} y
\end{array}\right)
$$

where $(x, y) \in \mathbb{K}^{2}$ has

$$
\|x\|^{2}+\|y\|^{2}=1 .
$$

These nontrivial projections all have rank 1, so they are the points of our would-be projective space. Our would-be projective space has just one line, corresponding to the projection 1 , and all the points lie on this line. It is easy to check that the axioms for a projective space hold. Since this projective space is 1-dimensional, we have succeeded in creating the projective line over $\mathbb{K}$. We call the set of points of this projective line $\mathbb{K} \mathbb{P}^{1}$.

Given any nonzero element $(x, y) \in \mathbb{K}^{2}$, we can normalize it and then use the above formula to get a point in $\mathbb{K P}^{1}$, which we call $[(x, y)]$. This allows us to describe $\mathbb{K} \mathbb{P}^{1}$ in terms of lines through the origin, as follows. Define an equivalence relation on nonzero elements of $\mathbb{K}^{2}$ by

$$
(x, y) \sim\left(x^{\prime}, y^{\prime}\right) \Longleftrightarrow[(x, y)]=\left[\left(x^{\prime}, y^{\prime}\right)\right] .
$$

We call an equivalence class for this relation a line through the origin in $\mathbb{K}^{2}$. We can then identify points in $\mathbb{K} \mathbb{P}^{1}$ with lines through the origin in $\mathbb{K}^{2}$.

Be careful: when $\mathbb{K}$ is the octonions, the line through the origin containing $(x, y)$ is not always equal to

$$
\{(\alpha x, \alpha y): \alpha \in \mathbb{K}\} .
$$

This is only true when $\mathbb{K}$ is associative, or when $x$ or $y$ is 1 . Luckily, we have $(x, y) \sim\left(y^{-1} x, 1\right)$ when $y \neq 0$ and $(x, y) \sim\left(1, x^{-1} y\right)$ when $x \neq 0$. Thus in either case we get a concrete description of the line through the origin containing $(x, y)$ : when $x \neq 0$ it equals

$$
\left\{\left(\alpha\left(y^{-1} x\right), \alpha\right): \alpha \in \mathbb{K}\right\}
$$

and when $y \neq 0$ it equals

$$
\left\{\left(\alpha, \alpha\left(x^{-1} y\right): \alpha \in \mathbb{K}\right\} .\right.
$$

In particular, the line through the origin containing $(x, y)$ is always a real vector space isomorphic to $\mathbb{K}$.

We can make $\mathbb{K} \mathbb{P}^{1}$ into a manifold as follows. By the above observations, we can cover it with two coordinate charts: one containing all points of the form $[(x, 1)]$, the other containing all points of the form $[(1, y)]$. It is easy to check that $[(x, 1)]=[(1, y)]$ iff $y=x^{-1}$, so the transition function from the first chart to the second is the map $x \mapsto x^{-1}$. Since this transition function and its inverse are smooth on the intersection of the two charts, $\mathbb{K}^{1}$ becomes a smooth manifold.

When pondering the geometry of projective lines it is handy to visualize the complex case, since $\mathbb{C P}^{1}$ is just the familiar 'Riemann sphere'. In this case, the map

$$
x \mapsto[(x, 1)]
$$


is given by stereographic projection:

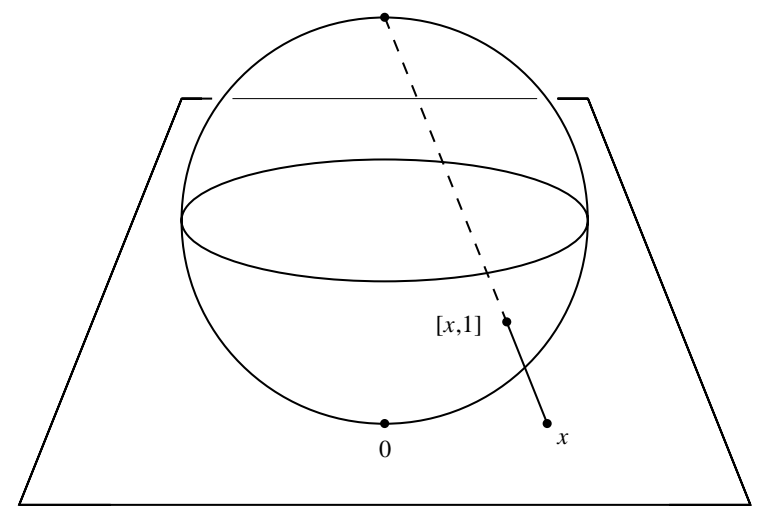

where we choose the sphere to have diameter 1 . This map from $\mathbb{C}$ to $\mathbb{C P}^{1}$ is one-toone and almost onto, missing only the point at infinity, or 'north pole'. Similarly, the map

$$
y \mapsto[(1, y)]
$$

misses only the south pole. Composing the first map with the inverse of the second, we get the map $x \mapsto x^{-1}$, which goes by the name of 'conformal inversion'. The southern hemisphere of the Riemann sphere consists of all points $[(x, 1)]$ with $\|x\| \leq$ 1 , while the northern hemisphere consists of all $[(1, y)]$ with $\|y\| \leq 1$. Unit complex numbers $x$ give points $[(x, 1)]=\left[\left(1, x^{-1}\right)\right]$ on the equator.

All these ideas painlessly generalize to $\mathbb{K P}^{1}$ for any normed division algebra $\mathbb{K}$. First of all, as a smooth manifold $\mathbb{K P}^{1}$ is just a sphere with dimension equal to that of $\mathbb{K}$ :

$$
\begin{aligned}
& \mathbb{R} \mathbb{P}^{1} \cong S^{1} \\
& \mathbb{C P}^{1} \cong S^{2} \\
& \mathbb{H} \mathbb{P}^{1} \cong S^{4} \\
& \mathbb{O P}^{1} \cong S^{8}
\end{aligned}
$$

We can think of it as the one-point compactification of $\mathbb{K}$. The 'southern hemisphere', 'northern hemisphere', and 'equator' of $\mathbb{K}$ have descriptions exactly like those given above for the complex case. Also, as in the complex case, the maps $x \mapsto[(x, 1)]$ and $y \mapsto[(1, y)]$ are angle-preserving with respect to the usual Euclidean metric on $\mathbb{K}$ and the round metric on the sphere.

One of the nice things about $\mathbb{K} \mathbb{P}^{1}$ is that it comes equipped with a vector bundle whose fiber over the point $[(x, y)]$ is the line through the origin corresponding to this point. This bundle is called the canonical line bundle, $L_{\mathbb{K}}$. Of course, when we are working with a particular division algebra, 'line' means a copy of this division algebra, so if we think of them as real vector bundles, $L_{\mathbb{R}}, L_{\mathbb{C}}, L_{\mathbb{H}}$ and $L_{\mathbb{O}}$ have dimensions $1,2,4$, and 8 , respectively.

These bundles play an important role in topology, so it is good to understand them in a number of ways. In general, any $k$-dimensional real vector bundle over $S^{n}$ can be formed by taking trivial bundles over the northern and southern hemispheres and gluing them together along the equator via a map $f: S^{n-1} \rightarrow \mathrm{O}(k)$. We must 
therefore be able to build the canonical line bundles $L_{\mathbb{R}}, L_{\mathbb{C}}, L_{\mathbb{H}}$ and $L_{\mathbb{O}}$ using maps

$$
\begin{array}{lll}
f_{\mathbb{R}}: & S^{0} \rightarrow \mathrm{O}(1) \\
f_{\mathbb{C}}: & S^{1} \rightarrow \mathrm{O}(2) \\
f_{\mathbb{H}}: & S^{3} \rightarrow \mathrm{O}(4) \\
f_{\mathbb{O}}: & S^{7} \rightarrow \mathrm{O}(8) .
\end{array}
$$

What are these maps? We can describe them all simultaneously. Suppose $\mathbb{K}$ is a normed division algebra of dimension $n$. In the southern hemisphere of $\mathbb{K P}^{1}$, we can identify any fiber of $L_{\mathbb{K}}$ with $\mathbb{K}$ by mapping the point $(\alpha x, \alpha)$ in the line $[(x, 1)]$ to the element $\alpha \in \mathbb{K}$. This trivializes the canonical line bundle over the southern hemisphere. Similarly, we can trivialize this bundle over the northern hemisphere by mapping the point $(\beta, \beta y)$ in the line $[(1, y)]$ to the element $\beta \in \mathbb{K}$. If $x \in \mathbb{K}$ has norm one, $[(x, 1)]=\left[\left(1, x^{-1}\right)\right]$ is a point on the equator, so we get two trivializations of the fiber over this point. These are related as follows: if $(\alpha x, \alpha)=\left(\beta, \beta x^{-1}\right)$, then $\beta=\alpha x$. The map $\alpha \mapsto \beta$ is thus right multiplication by $x$. In short,

$$
f_{\mathbb{K}}: S^{n-1} \rightarrow \mathrm{O}(n)
$$

is just the map sending any norm-one element $x \in \mathbb{K}$ to the operation of right multiplication by $x$.

If we take the homotopy class of the map $f_{\mathbb{K}}$, we obtain an element $\left[f_{\mathbb{K}}\right]$ in the homotopy group $\pi_{n-1}(\mathrm{O}(n))$. We shall not prove this here, but in every case this element happens to generate the relevant homotopy group! In other words:

- $\left[f_{\mathbb{R}}\right]$ generates $\pi_{0}(\mathrm{O}(1)) \cong \mathbb{Z}_{2}$.

- $\left[f_{\mathbb{C}}\right]$ generates $\pi_{1}(\mathrm{O}(2)) \cong \mathbb{Z}$.

- $\left[f_{\mathbb{H}}\right]$ generates $\pi_{3}(\mathrm{O}(4)) \cong \mathbb{Z}$.

- $\left[f_{\mathbb{O}}\right]$ generates $\pi_{7}(\mathrm{O}(8)) \cong \mathbb{Z}$.

Another nice perspective on the canonical line bundles $L_{\mathbb{K}}$ comes from looking at their unit sphere bundles. Any fiber of $L_{\mathbb{K}}$ is naturally an inner product space, since it is a line through the origin in $\mathbb{K}^{2}$. If we take the unit sphere in each fiber, we get a bundle of $(n-1)$-spheres over $\mathbb{K} \mathbb{P}^{1}$ called the Hopf bundle:

$$
p_{\mathbb{K}}: E_{\mathbb{K}} \rightarrow \mathbb{K \mathbb { P } ^ { 1 }}
$$

The projection $p_{\mathbb{K}}$ is called the Hopf map. The total space $E_{\mathbb{K}}$ consists of all the unit vectors in $\mathbb{K}^{2}$, so it is a sphere of dimension $2 n-1$. In short, the Hopf bundles look like this:

$$
\begin{aligned}
& \mathbb{K}=\mathbb{R}: \quad S^{0} \hookrightarrow S^{1} \quad \rightarrow \quad S^{1} \\
& \mathbb{K}=\mathbb{C}: \quad S^{1} \hookrightarrow S^{3} \quad \rightarrow S^{2} \\
& \mathbb{K}=\mathbb{H}: \quad S^{3} \hookrightarrow S^{7} \quad \rightarrow S^{4} \\
& \mathbb{K}=\mathbb{O}: \quad S^{7} \hookrightarrow S^{15} \quad \rightarrow \quad S^{8}
\end{aligned}
$$

We can understand the Hopf maps better by thinking about inverse images of points. The inverse image $p_{\mathbb{K}}^{-1}(x)$ of any point $x \in S^{n}$ is a $(n-1)$-sphere in $S^{2 n-1}$, and the inverse image of any pair of distinct points is a pair of linked spheres of this sort. When $\mathbb{K}=\mathbb{C}$ we get linked circles in $S^{3}$, which form the famous Hopf link: 


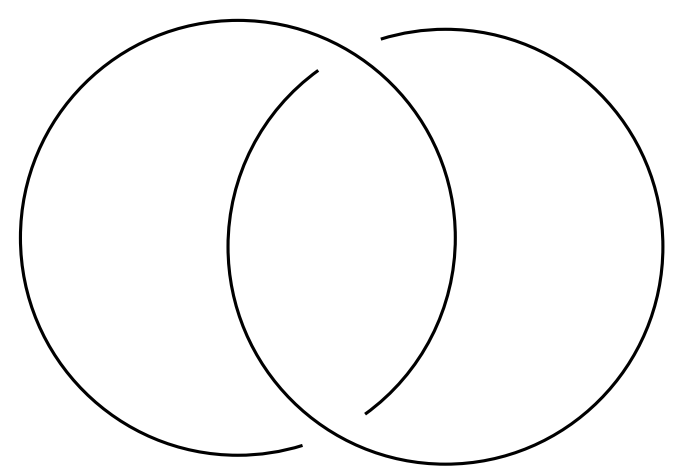

When $\mathbb{K}=\mathbb{O}$, we get a pair of linked 7-spheres in $S^{15}$.

To quantify this notion of linking, we can use the 'Hopf invariant'. Suppose for a moment that $n$ is any natural number greater than one, and let $f: S^{2 n-1} \rightarrow S^{n}$ be any smooth map. If $\omega$ is the normalized volume form on $S^{n}$, then $f^{*} \omega$ is a closed $n$-form on $S^{2 n-1}$. Since the $n$th cohomology of $S^{2 n-1}$ vanishes, $f^{*} \omega=d \alpha$ for some $(n-1)$-form $\alpha$. We define the Hopf invariant of $f$ to be the number

$$
H(f)=\int_{S^{2 n-1}} \alpha \wedge d \alpha
$$

This is easily seen to be invariant under smooth homotopies of the map $f$.

To see how the Hopf invariant is related to linking, we can compute it using homology rather than cohomology. If we take any two regular values of $f$, say $x$ and $y$, the inverse images of these points are compact oriented $(n-1)$-dimensional submanifolds of $S^{2 n-1}$. We can always find an oriented $n$-dimensional submanifold $X \subset S^{2 n-1}$ that has boundary equal to $f^{-1}(x)$ and that intersects $f^{-1}(y)$ transversely. The dimensions of $X$ and $f^{-1}(y)$ add up to $2 n-1$, so their intersection number is well-defined. By the duality between homology and cohomology, this number equals the Hopf invariant $H(f)$. This shows that the Hopf invariant is an integer. Moreover, it shows that when the Hopf invariant is nonzero, the inverse images of $x$ and $y$ are linked.

Using either of these approaches we can compute the Hopf invariant of $p_{\mathbb{C}}, p_{\mathbb{H}}$ and $p_{\mathbb{O}}$. They all turn out to have Hopf invariant 1 . This implies, for example, that the inverse images of distinct points under $p_{\mathbb{O}}$ are nontrivially linked 7 -spheres in $S^{15}$. It also implies that $p_{\mathbb{C}}, p_{\mathbb{H}}$ and $p_{\mathbb{O}}$ give nontrivial elements of $\pi_{2 n-1}\left(S^{n}\right)$ for $n=2,4$, and 8 . In fact, these elements generate the relevant homotopy groups! The real case is a bit different:

- $\left[p_{\mathbb{R}}\right]$ is twice the generator of $\pi_{1}\left(S^{1}\right) \cong \mathbb{Z}$.

- $\left[p_{\mathbb{C}}\right]$ generates $\pi_{3}\left(S^{2}\right) \cong \mathbb{Z}$.

- $\left[p_{\mathbb{H}}\right]$ generates $\pi_{7}\left(S^{3}\right) \cong \mathbb{Z}$.

- $\left[p_{\mathbb{O}}\right]$ generates $\pi_{15}\left(S^{7}\right) \cong \mathbb{Z}$.

A deep study of the Hopf invariant is one way to prove that any division algebra must have dimension 1, 2, 4 or 8 . One can show that if there exists an $n$-dimensional division algebra, then $S^{n-1}$ must be parallelizable: it must admit $n-1$ pointwise linearly independent smooth vector fields. One can also show that for $n>1, S^{n-1}$ is parallelizable iff there exists a map $f: S^{2 n-1} \rightarrow S^{n}$ with $H(f)=1$ [3, 11, 61]. The hard part is showing that a map from $S^{2 n-1}$ to $S^{n}$ can have Hopf invariant 1 only if $n=2,4$, or 8 . This was proved by Adams sometime about 1958 [1]. 
3.2. $\mathbb{O P}^{1}$ and Bott periodicity. We already touched upon Bott periodicity when we mentioned that the Clifford algebra $\mathrm{Cliff}_{n+8}$ is isomorphic to the algebra of $16 \times 16$ matrices with entries lying in $\mathrm{Cliff}_{n}$. This is but one of many related 'period8 ' phenomena that go by the name of Bott periodicity. The appearance of the number 8 here is no coincidence: all these phenomena are related to the octonions! Since this marvelous fact is somewhat underappreciated, it seems worthwhile to say a bit about it. Here we shall focus on those aspects that are related to $\mathbb{O P}^{1}$ and the canonical octonionic line bundle over this space.

Let us start with K-theory. This is a way of gaining information about a topological space by studying the vector bundles over it. If the space has holes in it, there will be nontrivial vector bundles that have 'twists' as we go around these holes. The simplest example is the 'Möbius strip' bundle over $S^{1}$, a 1-dimensional real vector bundle which has a $180^{\circ}$ twist as we go around the circle. In fact, this is just the canonical line bundle $L_{\mathbb{R}}$. The canonical line bundles $L_{\mathbb{C}}, L_{\mathbb{H}}$ and $L_{\mathbb{O}}$ provide higher-dimensional analogues of this example.

$\mathrm{K}$-theory tells us to study the vector bundles over a topological space $X$ by constructing an abelian group as follows. First, take the set consisting of all isomorphism classes of real vector bundles over $X$. Our ability to take direct sums of vector bundles gives this set an 'addition' operation making it into a commutative monoid. Next, adjoin formal 'additive inverses' for all the elements of this set, obtaining an abelian group. This group is called $K O(X)$, the real K-theory of $X$. Alternatively we could start with complex vector bundles and get a group called $K(X)$, but here we will be interested in real vector bundles.

Any real vector $E$ over $X$ gives an element $[E] \in K O(X)$, and these elements generate this group. If we pick a point in $X$, there is an obvious homomorphism $\operatorname{dim}: K O(X) \rightarrow \mathbb{Z}$ sending $[E]$ to the dimension of the fiber of $E$ at this point. Since the dimension is a rather obvious and boring invariant of vector bundles, it is nice to work with the kernel of this homomorphism, which is called the reduced real K-theory of $X$ and is denoted $\widetilde{K O}(X)$. This is an invariant of pointed spaces, i.e. spaces equipped with a designated point or basepoint.

Any sphere becomes a pointed space if we take the north pole as basepoint. The reduced real K-theory of the first eight spheres looks like this:

$$
\begin{aligned}
& \widetilde{K O}\left(S^{1}\right) \cong \mathbb{Z}_{2} \\
& \widetilde{K O}\left(S^{2}\right) \cong \mathbb{Z}_{2} \\
& \widetilde{K O}\left(S^{3}\right) \cong 0 \\
& \widetilde{K O}\left(S^{4}\right) \cong \mathbb{Z} \\
& \widetilde{K O}\left(S^{5}\right) \cong 0 \\
& \widetilde{K O}\left(S^{6}\right) \cong 0 \\
& \widetilde{K O}\left(S^{7}\right) \cong 0 \\
& \widetilde{K O}\left(S^{8}\right) \cong \mathbb{Z}
\end{aligned}
$$

where, as one might guess,

- $\left[L_{\mathbb{R}}\right]$ generates $\widetilde{K O}\left(S^{1}\right)$.

- $\left[L_{\mathbb{C}}\right]$ generates $\widetilde{K O}\left(S^{2}\right)$. 
- $\left[L_{\mathbb{H}}\right]$ generates $\widetilde{K O}\left(S^{4}\right)$.

- $\left[L_{\mathbb{O}}\right]$ generates $\widetilde{K O}\left(S^{8}\right)$.

As mentioned in the previous section, one can build any $k$-dimensional real vector bundle over $S^{n}$ using a map $f: S^{n-1} \rightarrow \mathrm{O}(k)$. In fact, isomorphism classes of such bundles are in one-to-one correspondence with homotopy classes of such maps. Moreover, two such bundles determine the same element of $\widetilde{K O}(X)$ if and only if the corresponding maps become homotopy equivalent after we compose them with the inclusion $\mathrm{O}(k) \hookrightarrow \mathrm{O}(\infty)$, where $\mathrm{O}(\infty)$ is the direct limit of the groups $\mathrm{O}(k)$. It follows that

$$
\widetilde{K O}\left(S^{n}\right) \cong \pi_{n-1}(\mathrm{O}(\infty)) \text {. }
$$

This fact gives us the list of homotopy groups of $\mathrm{O}(\infty)$ which appears in the Introduction. It also means that to prove Bott periodicity for these homotopy groups:

$$
\pi_{i+8}(\mathrm{O}(\infty)) \cong \pi_{i}(\mathrm{O}(\infty)),
$$

it suffices to prove Bott periodicity for real K-theory:

$$
\widetilde{K O}\left(S^{n+8}\right) \cong \widetilde{K O}\left(S^{n}\right) .
$$

Why do we have Bott periodicity in real K-theory? It turns out that there is a graded ring $K O$ with

$$
K O_{n}=\widetilde{K O}\left(S^{n}\right) .
$$

The product in this ring comes from our ability to take 'smash products' of spheres and also of real vector bundles over these spheres. Multiplying by $\left[L_{\mathbb{O}}\right]$ gives an isomorphism

$$
\begin{array}{ccc}
\widetilde{K O}\left(S^{n}\right) & \rightarrow \widetilde{K O}\left(S^{n+8}\right) \\
x & \mapsto & {\left[L_{\mathbb{O}}\right] x .}
\end{array}
$$

In other words, the canonical octonionic line bundle over $\mathbb{O P}^{1}$ generates Bott periodicity!

There is much more to say about this fact and how it relates to Bott periodicity for Clifford algebras, but alas, this would take us too far afield. We recommend that the interested reader turn to some introductory texts on K-theory, for example the one by Dale Husemoller [55. Unfortunately, all the books I know downplay the role of the octonions. To spot it, one must bear in mind the relation between the octonions and Clifford algebras, discussed in Section 2.3 above.

3.3. $\mathbb{O P}^{1}$ and Lorentzian geometry. In Section 3.1 we sketched a systematic approach to projective lines over the normed division algebras. The most famous example is the Riemann sphere, $\mathbb{C P}^{1}$. As emphasized by Penrose [76, this space has a fascinating connection to Lorentzian geometry - or in other words, special relativity. All conformal transformations of the Riemann sphere come from fractional linear transformations

$$
z \mapsto \frac{a z+b}{c z+d}, \quad a, b, c, d \in \mathbb{C} .
$$

It is easy to see that the group of such transformations is isomorphic to $\operatorname{PSL}(2, \mathbb{C})$ : $2 \times 2$ complex matrices with determinant 1 , modulo scalar multiples of the identity. Less obviously, it is also isomorphic to the Lorentz group $\mathrm{SO}_{0}(3,1)$ : the identity 
component of the group of linear transformations of $\mathbb{R}^{4}$ that preserve the Minkowski metric

$$
x \cdot y=x_{1} y_{1}+x_{2} y_{2}+x_{3} y_{3}-x_{4} y_{4} .
$$

This fact has a nice explanation in terms of the 'heavenly sphere'. Mathematically, this is the 2-sphere consisting of all lines of the form $\{\alpha x\}$ where $x \in \mathbb{R}^{4}$ has $x \cdot x=0$. In special relativity such lines represent light rays, so the heavenly sphere is the sphere on which the stars appear to lie when you look at the night sky. This sphere inherits a conformal structure from the Minkowski metric on $\mathbb{R}^{4}$. This allows us to identify the heavenly sphere with $\mathbb{C P}^{1}$, and it implies that the Lorentz group acts as conformal transformations of $\mathbb{C P}^{1}$. In concrete terms, what this means is that if you shoot past the earth at nearly the speed of light, the constellations in the sky will appear distorted, but all angles will be preserved.

In fact, these results are not special to the complex case: the same ideas work for the other normed division algebras as well! The algebras $\mathbb{R}, \mathbb{C}, \mathbb{H}$ and $\mathbb{O}$ are related to Lorentzian geometry in 3, 4, 6, and 10 dimensions, respectively [67], [68], 69], [84], [92]. Even better, a full explanation of this fact brings out new relationships between the normed division algebras and spinors. In what follows we explain how this works for all 4 normed division algebras, with special attention to the peculiarities of the octonionic case.

To set the stage, we first recall the most mysterious of the four infinite series of Jordan algebras listed at the beginning of Section 3 the spin factors. We described these quite concretely, but a more abstract approach brings out their kinship to Clifford algebras. Given an $n$-dimensional real inner product space $V$, let the spin factor $\mathrm{J}(V)$ be the Jordan algebra freely generated by $V$ modulo relations

$$
v^{2}=\|v\|^{2}
$$

Polarizing and applying the commutative law, we obtain

$$
v \circ w=\langle v, w\rangle
$$

so $\mathrm{J}(V)$ is isomorphic to $V \oplus \mathbb{R}$ with the product

$$
(v, \alpha) \circ(w, \beta)=(\alpha w+\beta v,\langle v, w\rangle+\alpha \beta) .
$$

Though Jordan algebras were invented to study quantum mechanics, the spin factors are also deeply related to special relativity. We can think of $\mathrm{J}(V) \cong V \oplus \mathbb{R}$ as Minkowksi spacetime, with $V$ as space and $\mathbb{R}$ as time. The reason is that $\mathrm{J}(V)$ is naturally equipped with a symmetric bilinear form of signature $(n, 1)$, the Minkowski metric:

$$
(v, \alpha) \cdot(w, \beta)=\langle v, w\rangle-\alpha \beta .
$$

The group of linear transformations preserving the Minkowski metric is called $\mathrm{O}(n, 1)$, and the identity component of this is called the Lorentz group, $\mathrm{SO}_{0}(n, 1)$. We define the lightcone $\mathrm{C}(V)$ to consist of all nonzero $x \in \mathrm{J}(V)$ with $x \cdot x=0$. A 1-dimensional subspace of $\mathrm{J}(V)$ spanned by an element of the lightcone is called a light ray, and the space of all light rays is called the heavenly sphere $\mathrm{S}(V)$. We can identify the heavenly sphere with the unit sphere in $V$, since every light ray is spanned by an element of the form $(v, 1)$ where $v \in V$ has norm one. Here is a picture of the lightcone and the heavenly sphere when $V$ is 2-dimensional: 


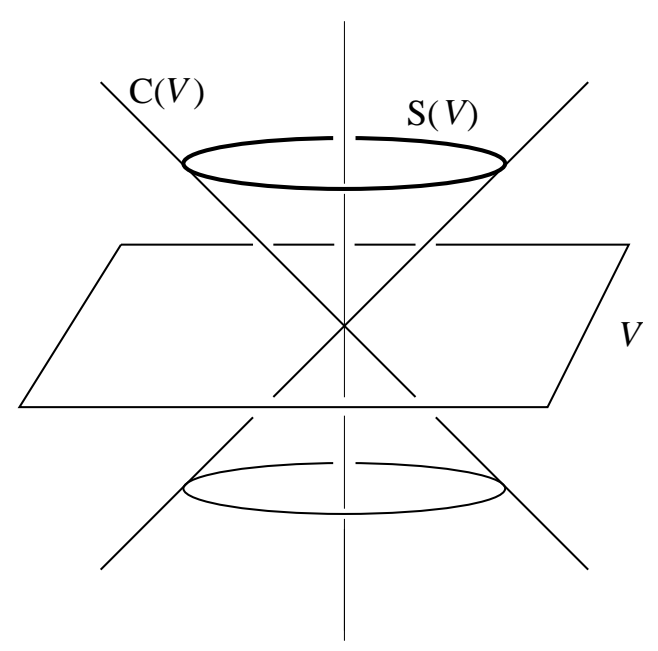

When $V$ is at least 2-dimensional, we can build a projective space from the Jordan algebra $\mathrm{J}(V)$. The result is none other than the heavenly sphere! To see this, note that aside from the elements 0 and 1 , all projections in $\mathrm{J}(V)$ are of the form $p=\frac{1}{2}(v, 1)$ where $v \in V$ has norm one. These are the points of our projective space, but as we have seen, they also correspond to points of the heavenly sphere. Our projective space has just one line, corresponding to the projection $1 \in \mathrm{J}(V)$. We can visualize this line as the heavenly sphere itself.

What does all this have to do with normed division algebras? To answer this, let $\mathbb{K}$ be a normed division algebra of dimension $n$. Then the Jordan algebra $h_{2}(\mathbb{K})$ is secretly a spin factor! There is an isomorphism

$$
\phi: \mathfrak{h}_{2}(\mathbb{K}) \rightarrow J(\mathbb{K} \oplus \mathbb{R}) \cong \mathbb{K} \oplus \mathbb{R} \oplus \mathbb{R}
$$

given by

$$
\phi\left(\begin{array}{cc}
\alpha+\beta & x \\
x^{*} & \alpha-\beta
\end{array}\right)=(x, \beta, \alpha), \quad x \in \mathbb{K}, \alpha, \beta \in \mathbb{R} .
$$

Furthermore, the determinant of matrices in $\mathfrak{h}_{2}(\mathbb{K})$ is well-defined even when $\mathbb{K}$ is noncommutative or nonassociative:

$$
\operatorname{det}\left(\begin{array}{cc}
\alpha+\beta & x \\
x^{*} & \alpha-\beta
\end{array}\right)=\alpha^{2}-\beta^{2}-\|x\|^{2},
$$

and clearly we have

$$
\operatorname{det}(a)=-\phi(a) \cdot \phi(a)
$$

for all $a \in \mathfrak{h}_{2}(\mathbb{K})$.

These facts have a number of nice consequences. First of all, since the Jordan algebras $J(\mathbb{K} \oplus \mathbb{R})$ and $\mathfrak{h}_{2}(\mathbb{K})$ are isomorphic, so are their associated projective spaces. We have seen that the former space is the heavenly sphere $S(\mathbb{K} \oplus \mathbb{R})$, and that the latter is $\mathbb{K \mathbb { P } ^ { 1 }}$. It follows that

$$
\mathbb{K} \mathbb{P}^{1} \cong \mathrm{S}(\mathbb{K} \oplus \mathbb{R}) .
$$

This gives another proof of something we already saw in Section $3.1 \quad \mathbb{K} \mathbb{P}^{1}$ is an $n$-sphere. But it shows more. The Lorentz group $\mathrm{SO}_{0}(n+1,1)$ has an obvious action on the heavenly sphere, and the usual conformal structure on the sphere 
is invariant under this action. Using the above isomorphism we can transfer this group action and invariant conformal structure to $\mathbb{K} \mathbb{P}^{1}$ in a natural way.

Secondly, it follows that the determinant-preserving linear transformations of $\mathfrak{h}_{2}(\mathbb{K})$ form a group isomorphic to $\mathrm{O}(n+1,1)$. How can we find some transformations of this sort? If $\mathbb{K}=\mathbb{R}$, this is easy: when $g \in \mathrm{SL}(2, \mathbb{R})$ and $x \in \mathfrak{h}_{2}(\mathbb{R})$, we again have $g x g^{*} \in \mathfrak{h}_{2}(\mathbb{R})$, and

$$
\operatorname{det}\left(g x g^{*}\right)=\operatorname{det}(x) .
$$

This gives a homomorphism from $\mathrm{SL}(2, \mathbb{R})$ to $\mathrm{O}(2,1)$. This homomorphism is twoto-one, since both $g=1$ and $g=-1$ act trivially, and it maps $\operatorname{SL}(2, \mathbb{R})$ onto the identity component of $\mathrm{O}(2,1)$. It follows that $\mathrm{SL}(2, \mathbb{R})$ is a double cover of $\mathrm{SO}_{0}(2,1)$. The exact same construction works for $\mathbb{K}=\mathbb{C}$, so $\mathrm{SL}(2, \mathbb{C})$ is a double cover of $\mathrm{SO}_{0}(3,1)$.

For the other two normed division algebras the above calculation involving determinants breaks down, and it even becomes tricky to define the group $\operatorname{SL}(2, \mathbb{K})$, so we start by working at the Lie algebra level. We say an $m \times m$ matrix with entries in the normed division algebra $\mathbb{K}$ is traceless if the sum of its diagonal entries is zero. Any such traceless matrix acts as a real-linear operator on $\mathbb{K}^{m}$. When $\mathbb{K}$ is commutative and associative, the space of operators coming from $m \times m$ traceless matrices with entries in $\mathbb{K}$ is closed under commutators, but otherwise it is not, so we define $\mathfrak{s l}(m, \mathbb{K})$ to be the Lie algebra of operators on $\mathbb{K}^{m}$ generated by operators of this form. This Lie algebra in turn generates a Lie group of real-linear operators on $\mathbb{K}^{m}$, which we call $\mathrm{SL}(m, \mathbb{K})$. Note that multiplication in this group is given by composition of real-linear operators, which is associative even for $\mathbb{K}=\mathbb{O}$.

The Lie algebra $\mathfrak{s l}(m, \mathbb{K})$ comes born with a representation: its fundamental representation as real-linear operators on $\mathbb{K}^{m}$, given by

$$
a: x \mapsto a x, \quad x \in \mathbb{K}^{m}
$$

whenever $a \in \mathfrak{s l}(m, \mathbb{K})$ actually corresponds to a traceless $m \times m$ matrix with entries in $\mathbb{K}$. Tensoring the fundamental representation with its dual, we get a representation of $\mathfrak{s l}(m, \mathbb{K})$ on the space of matrices $\mathbb{K}[m]$, given by

$$
a: x \mapsto a x+x a^{*}, \quad x \in \mathbb{K}[m]
$$

whenever $a$ is a traceless matrix with entries in $\mathbb{K}$. Since $a x+x a^{*}$ is hermitian whenever $x$ is, this representation restricts to a representation of $\mathfrak{s l}(m, \mathbb{K})$ on $\mathfrak{h}_{m}(\mathbb{K})$. This in turn can be exponentiated to obtain a representation of the group $\mathrm{SL}(m, \mathbb{K})$ on $\mathfrak{h}_{m}(\mathbb{K})$.

Now let us return to the case $m=2$. One can prove that the representation of $\mathrm{SL}(2, \mathbb{K})$ on $\mathfrak{h}_{2}(\mathbb{K})$ is determinant-preserving simply by checking that

$$
\left.\frac{d}{d t} \operatorname{det}\left(x+t\left(a x+x a^{*}\right)\right)\right|_{t=0}=0
$$

when $x$ lies in $\mathfrak{h}_{2}(\mathbb{K})$ and $a \in \mathbb{K}[2]$ is traceless. Here the crucial thing is to make sure that the calculation is not spoiled by noncommutativity or nonassociativity. It follows that we have a homomorphism

$$
\alpha_{\mathbb{K}}: \mathrm{SL}(2, \mathbb{K}) \rightarrow \mathrm{SO}_{0}(n+1,1) .
$$

One can check that this is onto and that its kernel consists of the matrices \pm 1 . Thus if we define

$$
\operatorname{PSL}(2, \mathbb{K})=\mathrm{SL}(2, \mathbb{K}) /\{ \pm 1\}
$$


we get isomorphisms

$$
\begin{aligned}
& \operatorname{PSL}(2, \mathbb{R}) \cong \mathrm{SO}_{0}(2,1) \\
& \operatorname{PSL}(2, \mathbb{C}) \cong \mathrm{SO}_{0}(3,1) \\
& \operatorname{PSL}(2, \mathbb{H}) \cong \mathrm{SO}_{0}(6,1) \\
& \operatorname{PSL}(2, \mathbb{O}) \cong \mathrm{SO}_{0}(9,1) .
\end{aligned}
$$

Putting this together with our earlier observations, it follows that $\operatorname{PSL}(2, \mathbb{K})$ acts as conformal transformations of $\mathbb{K P}^{1}$.

We conclude with some words about how all this relates to spinors. The machinery of Clifford algebras and spinors extends effortlessly from the case of inner product spaces to vector spaces equipped with an indefinite metric. In particular, the Lorentz group $\mathrm{SO}_{0}(n+1,1)$ has a double cover called $\operatorname{Spin}(n+1,1)$, and this group has certain representations called spinor representations. When $n=1,2,4$ or 8 , we actually have

$$
\operatorname{Spin}(n+1,1) \cong \mathrm{SL}(2, \mathbb{K})
$$

where $\mathbb{K}$ is the normed division algebra of dimension $n$. The fundamental representation of $\operatorname{SL}(2, \mathbb{K})$ on $\mathbb{K}^{2}$ is the left-handed spinor representation of $\operatorname{Spin}(n+1,1)$. Its dual is the right-handed spinor representation. Moreover, the interaction between vectors and spinors that serves as the basis of supersymmetric theories of physics in spacetimes of dimension $3,4,6$ and 10 is just the action of $\mathfrak{h}_{2}(\mathbb{K})$ on $\mathbb{K}^{2}$ by matrix multiplication. In a Feynman diagram, this is represented as follows:

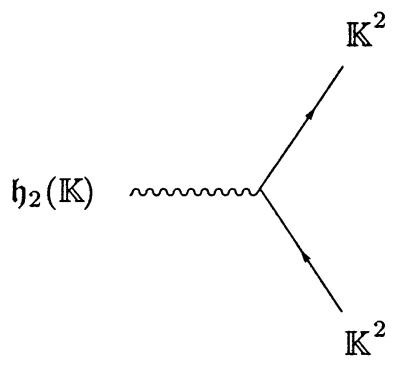

In the case $\mathbb{K}=\mathbb{C}$, Penrose [76] has described a nice trick for getting points on the heavenly sphere from spinors. In fact, it also works for other normed division algebras: if $(x, y) \in \mathbb{K}^{2}$ is nonzero, the hermitian matrix

$$
\left(\begin{array}{l}
x \\
y
\end{array}\right)\left(\begin{array}{ll}
x^{*} & y^{*}
\end{array}\right)=\left(\begin{array}{ll}
x x^{*} & x y^{*} \\
y x^{*} & y y^{*}
\end{array}\right)
$$

is nonzero but has determinant zero, so it defines a point on the heavenly sphere. If we restrict to spinors of norm one, this trick reduces to the Hopf map. Moreover, it clarifies the curious double role of $\mathbb{K P}^{1}$ as both the heavenly sphere in special relativity and a space of propositions in the quantum logic associated to the Jordan algebra $\mathfrak{h}_{2}(\mathbb{K})$ : any point on the heavenly sphere corresponds to a proposition specifying the state of a spinor!

3.4. $\mathbb{O P}^{2}$ and the exceptional Jordan algebra. The octonions are fascinating in themselves, but the magic really starts when we use them to construct the exceptional Jordan algebra $\mathfrak{h}_{3}(\mathbb{O})$ and its associated projective space, the octonionic 
projective plane. The symmetry groups of these structures turn out to be exceptional Lie groups, and triality gains an eerie pervasive influence over the proceedings, since an element of $\mathfrak{h}_{3}(\mathbb{O})$ consists of 3 octonions and 3 real numbers. Using the relation between normed division algebras and trialities, we get an isomorphism

$$
\begin{aligned}
& \mathfrak{h}_{3}(\mathbb{O}) \quad \cong \mathbb{R}^{3} \oplus V_{8} \oplus S_{8}^{+} \oplus S_{8}^{-} \\
& \left(\begin{array}{ccc}
\alpha & z^{*} & y^{*} \\
z & \beta & x \\
y & x^{*} & \gamma
\end{array}\right) \mapsto \quad((\alpha, \beta, \gamma), x, y, z)
\end{aligned}
$$

where $\alpha, \beta, \gamma \in \mathbb{R}$ and $x, y, z \in \mathbb{O}$. Examining the Jordan product in $\mathfrak{h}_{3}(\mathbb{O})$ then reveals a wonderful fact: while superficially this product is defined using the *algebra structure of $\mathbb{O}$, it can actually be defined using only the natural maps

$$
V_{8} \times S_{8}^{+} \rightarrow S_{8}^{-}, \quad V_{8} \times S_{8}^{-} \rightarrow S_{8}^{+}, \quad S_{8}^{+} \times S_{8}^{-} \rightarrow V_{8}
$$

together with the inner products on these 3 spaces. All this information is contained in the normed triality

$$
t_{8}: V_{8} \times S_{8}^{+} \times S_{8}^{-} \rightarrow \mathbb{R},
$$

so any automorphism of this triality gives a automorphism of $\mathfrak{h}_{3}(\mathbb{O})$. In Section 2.4 we saw that $\operatorname{Aut}\left(t_{8}\right) \cong \operatorname{Spin}(8)$. With a little thought, it follows that

$$
\operatorname{Spin}(8) \subseteq \operatorname{Aut}\left(\mathfrak{h}_{3}(\mathbb{O})\right) .
$$

However, this picture of $\mathfrak{h}_{3}(\mathbb{O})$ in terms of 8-dimensional Euclidean geometry is just part of a bigger picture - a picture set in 10-dimensional Minkowski spacetime! If we regard $\mathfrak{h}_{2}(\mathbb{O})$ as sitting in the lower right-hand corner of $\mathfrak{h}_{3}(\mathbb{O})$, we get an isomorphism

$$
\begin{aligned}
\mathfrak{h}_{3}(\mathbb{O}) & \cong \quad \mathbb{R} \oplus \mathfrak{h}_{2}(\mathbb{O}) \oplus \mathbb{O}^{2} \\
\left(\begin{array}{cc}
\alpha & \psi^{*} \\
\psi & a
\end{array}\right) & \mapsto \quad(\alpha, a, \psi) .
\end{aligned}
$$

We saw in Section 3.3 that $a \in \mathfrak{h}_{2}(\mathbb{O})$ and $\psi \in \mathbb{O}^{2}$ can be identified with a vector and a spinor in 10-dimensional Minkowski spacetime, respectively. Similarly, $\alpha$ is a scalar.

This picture gives a representation of $\operatorname{Spin}(9,1)$ as linear transformations of $\mathfrak{h}_{3}(\mathbb{O})$. Unfortunately, most of these transformations do not preserve the Jordan product on $\mathfrak{h}_{3}(\mathbb{O})$. As we shall see, they only preserve a lesser structure on $\mathfrak{h}_{3}(\mathbb{O})$ : the determinant. However, the transformations coming from the subgroup $\operatorname{Spin}(9) \subset \operatorname{Spin}(9,1)$ do preserve the Jordan product. We can see this as follows. As a representation of $\operatorname{Spin}(9), \mathfrak{h}_{2}(\mathbb{O})$ splits into 'space' and 'time':

$$
\mathfrak{h}_{2}(\mathbb{O}) \cong V_{9} \oplus \mathbb{R}
$$

with the two pieces corresponding to the traceless elements of $\mathfrak{h}_{2}(\mathbb{O})$ and the real multiples of the identity, respectively. On the other hand, the spinor representation of $\mathfrak{s o}(9)$ splits as $S_{8}^{+} \oplus S_{8}^{-}$when we restrict it to $\mathfrak{s o}(8)$, so we have

$$
\mathbb{O}^{2} \cong S_{9} \text {. }
$$


We thus obtain an isomorphism

$$
\begin{aligned}
& \mathfrak{h}_{3}(\mathbb{O}) \quad \cong \mathbb{R}^{2} \oplus V_{9} \oplus S_{9} \\
& \left(\begin{array}{cc}
\alpha & \psi^{*} \\
\psi & a+\beta
\end{array}\right) \mapsto \quad((\alpha, \beta), a, \psi)
\end{aligned}
$$

where $a \in \mathfrak{h}_{2}(\mathbb{O})$ has vanishing trace and $\beta$ is a real multiple of the identity. In these terms, one can easily check that the Jordan product in $\mathfrak{h}_{3}(\mathbb{O})$ is built from invariant operations on scalars, vectors and spinors in 9 dimensions. It follows that

$$
\operatorname{Spin}(9) \subseteq \operatorname{Aut}\left(\mathfrak{h}_{3}(\mathbb{O})\right) .
$$

For more details on this, see Harvey's book [53].

This does not exhaust all the symmetries of $\mathfrak{h}_{3}(\mathbb{O})$, since there are other automorphisms coming from the permutation group on 3 letters which acts on $(\alpha, \beta, \gamma) \in \mathbb{R}^{3}$ and $(x, y, z) \in \mathbb{O}^{3}$ in an obvious way. Also, any matrix $g \in \mathrm{O}(3)$ acts by conjugation as an automorphism of $\mathfrak{h}_{3}(\mathbb{O})$; since the entries of $g$ are real, there is no problem with nonassociativity here. The group $\operatorname{Spin}(9)$ is 36 -dimensional, but the full automorphism group $\mathfrak{h}_{3}(\mathbb{O})$ is much bigger: it is 52 -dimensional. As we explain in Section 4.2 it goes by the name of $\mathrm{F}_{4}$.

However, we can already do something interesting with the automorphisms we have: we can use them to diagonalize any element of $\mathfrak{h}_{3}(\mathbb{O})$. To see this, first note that the rotation group, and thus Spin(9), acts transitively on the unit sphere in $V_{9}$. This means we can use an automorphism in our Spin(9) subgroup to bring any element of $\mathfrak{h}_{3}(\mathbb{O})$ to the form

$$
\left(\begin{array}{ccc}
\alpha & z^{*} & y^{*} \\
z & \beta & x \\
y & x^{*} & \gamma
\end{array}\right)
$$

where $x$ is real. The next step is to apply an automorphism that makes $y$ and $z$ real while leaving $x$ alone. To do this, note that the subgroup of Spin(9) fixing any nonzero vector in $V_{9}$ is isomorphic to $\operatorname{Spin}(8)$. When we restrict the representation $S_{9}$ to this subgroup, it splits as $S_{8}^{+} \oplus S_{8}^{-}$, and with some work [53] one can show that $\operatorname{Spin}(8)$ acts on $S_{8}^{+} \oplus S_{8}^{-} \cong \mathbb{O}^{2}$ in such a way that any element $(y, z) \in \mathbb{O}^{2}$ can be carried to an element with both components real. The final step is to take our element of $\mathfrak{h}_{3}(\mathbb{O})$ with all real entries and use an automorphism to diagonalize it. We can do this by conjugating it with a suitable matrix in $\mathrm{O}(3)$.

To understand $\mathbb{O P}^{2}$, we need to understand projections in $\mathfrak{h}_{3}(\mathbb{O})$. Here is where our ability to diagonalize matrices in $\mathfrak{h}_{3}(\mathbb{O})$ via automorphisms comes in handy. Up to automorphism, every projection in $\mathfrak{h}_{3}(\mathbb{O})$ looks like one of these four:

$$
\begin{array}{ll}
p_{0}=\left(\begin{array}{lll}
0 & 0 & 0 \\
0 & 0 & 0 \\
0 & 0 & 0
\end{array}\right), & p_{1}=\left(\begin{array}{lll}
1 & 0 & 0 \\
0 & 0 & 0 \\
0 & 0 & 0
\end{array}\right), \\
p_{2}=\left(\begin{array}{lll}
1 & 0 & 0 \\
0 & 1 & 0 \\
0 & 0 & 0
\end{array}\right), & p_{3}=\left(\begin{array}{lll}
1 & 0 & 0 \\
0 & 1 & 0 \\
0 & 0 & 1
\end{array}\right) .
\end{array}
$$

Now, the trace of a matrix in $\mathfrak{h}_{3}(\mathbb{O})$ is invariant under automorphisms, because we can define it using only the Jordan algebra structure:

$$
\operatorname{tr}(a)=\frac{1}{9} \operatorname{tr}\left(L_{a}\right), \quad a \in \mathfrak{h}_{3}(\mathbb{O})
$$


where $L_{a}$ is left multiplication by $a$. It follows that the trace of any projection in $\mathfrak{h}_{3}(\mathbb{O})$ is $0,1,2$, or 3 . Furthermore, the rank of any projection $p \in \mathfrak{h}_{3}(\mathbb{O})$ equals its trace. To see this, first note that $\operatorname{tr}(p) \geq \operatorname{rank}(p)$, since $p<q$ implies $\operatorname{tr}(p)<\operatorname{tr}(q)$, and the trace goes up by integer steps. Thus we only need to show $\operatorname{tr}(p) \leq \operatorname{rank}(p)$. For this it suffices to consider the four projections shown above, as both trace and rank are invariant under automorphisms. Since $p_{0}<p_{1}<p_{2}<p_{3}$, it is clear that for these projections we indeed have $\operatorname{tr}(p) \leq \operatorname{rank}(p)$.

It follows that the points of the octonionic projective plane are projections with trace 1 in $\mathfrak{h}_{3}(\mathbb{O})$, while the lines are projections with trace 2. A calculation 53 shows that any projection with trace 1 has the form

$$
p=\left(\begin{array}{l}
x \\
y \\
z
\end{array}\right)\left(\begin{array}{lll}
x^{*} & y^{*} & z^{*}
\end{array}\right)=\left(\begin{array}{lll}
x x^{*} & x y^{*} & x z^{*} \\
y x^{*} & y y^{*} & y z^{*} \\
z x^{*} & z y^{*} & z z^{*}
\end{array}\right)
$$

where $(x, y, z) \in \mathbb{O}^{3}$ has

$$
(x y) z=x(y z), \quad\|x\|^{2}+\|y\|^{2}+\|z\|^{2}=1 .
$$

On the other hand, any projection with trace 2 is of the form $1-p$ where $p$ has trace 1. This sets up a one-to-one correspondence between points and lines in the octonionic projective plane. If we use this correspondence to think of both as trace- 1 projections, the point $p$ lies on the line $p^{\prime}$ if and only if $p<1-p^{\prime}$. Of course, $p<1-p^{\prime}$ iff $p^{\prime}<1-p$. The symmetry of this relation means the octonionic projective plane is self-dual! This is also true of the real, complex and quaternionic projective planes. In all cases, the operation that switches points and lines corresponds in quantum logic to the 'negation' of propositions 97.

We use $\mathbb{O P}^{2}$ to stand for the set of points in the octonionic projective plane. Given any nonzero element $(x, y, z) \in \mathbb{O}^{3}$ with $(x y) z=x(y z)$, we can normalize it and then use equation (10) to obtain a point $[(x, y, z)] \in \mathbb{O P}^{2}$. Copying the strategy that worked for $\mathbb{O P}^{1}$, we can make $\mathbb{O P}^{2}$ into a smooth manifold by covering it with three coordinate charts:

- one chart containing all points of the form $[(x, y, 1)]$,

- one chart containing all points of the form $[(x, 1, z)]$,

- one chart containing all points of the form $[(1, y, z)]$.

Checking that this works is a simple calculation. The only interesting part is to make sure that whenever the associative law might appear necessary, we can either use the alternativity of the octonions or the fact that only triples with $(x y) z=x(y z)$ give points $[(x, y, z)] \in \mathbb{O} \mathbb{P}^{2}$.

We thus obtain the following picture of the octonionic projective plane. As a manifold, $\mathbb{O P}^{2}$ is 16 -dimensional. The lines in $\mathbb{O P}^{2}$ are copies of $\mathbb{O P}^{1}$, and thus 8 -spheres. For any two distinct points in $\mathbb{O P}^{2}$, there is a unique line on which they both lie. For any two distinct lines, there is a unique point lying on both of them. There is a 'duality' transformation that maps points to lines and vice versa while preserving this incidence relation. In particular, since the space of all points lying on any given line is a copy of $\mathbb{O P}^{1}$, so is the space of all lines containing a given point!

To dig more deeply into the geometry of $\mathbb{O P}^{2}$ one needs another important structure on the exceptional Jordan algebra: the determinant. We saw in Section 3.3 that despite noncommutativity and nonassociativity, the determinant of a matrix 
in $\mathfrak{h}_{2}(\mathbb{O})$ is a well-defined and useful concept. The same holds for $\mathfrak{h}_{3}(\mathbb{O})$ ! We can define the determinant of a matrix in $\mathfrak{h}_{3}(\mathbb{O})$ by

$$
\operatorname{det}\left(\begin{array}{ccc}
\alpha & z^{*} & y^{*} \\
z & \beta & x \\
y & x^{*} & \gamma
\end{array}\right)=\alpha \beta \gamma-\left(\alpha\|x\|^{2}+\beta\|y\|^{2}+\gamma\|z\|^{2}\right)+2 \operatorname{Re}(x y z) .
$$

We can express this in terms of the trace and product via

$$
\operatorname{det}(a)=\frac{1}{3} \operatorname{tr}\left(a^{3}\right)-\frac{1}{2} \operatorname{tr}\left(a^{2}\right) \operatorname{tr}(a)+\frac{1}{6} \operatorname{tr}(a)^{3} .
$$

This shows that the determinant is invariant under all automorphisms of $\mathfrak{h}_{3}(\mathbb{O})$. However, the determinant is invariant under an even bigger group of linear transformations. As we shall see in Section 4.4 this group is 78-dimensional: it is a noncompact real form of the exceptional Lie group $\mathrm{E}_{6}$. This extra symmetry makes it worth seeing how much geometry we can do starting with just the determinant and the vector space structure of $\mathfrak{h}_{3}(\mathbb{O})$.

The determinant is a cubic form on $\mathfrak{h}_{3}(\mathbb{O})$, so there is a unique trilinear form

$$
(\cdot, \cdot, \cdot): \mathfrak{h}_{3}(\mathbb{O}) \times \mathfrak{h}_{3}(\mathbb{O}) \times \mathfrak{h}_{3}(\mathbb{O}) \rightarrow \mathbb{R}
$$

such that

$$
(a, a, a)=\operatorname{det}(a) .
$$

By dualizing this, we obtain the so-called cross product

$$
\times: \mathfrak{h}_{3}(\mathbb{O}) \times \mathfrak{h}_{3}(\mathbb{O}) \rightarrow \mathfrak{h}_{3}(\mathbb{O})^{*} .
$$

Explicitly, this is given by

$$
(a \times b)(c)=(a, b, c) .
$$

Despite its name, this product is commutative.

We have already seen that points of $\mathbb{O P}^{2}$ correspond to trace-1 projections in $\mathfrak{h}_{3}(\mathbb{O})$. Freudenthal [39] noticed that these are the same as elements $p \in \mathfrak{h}_{3}(\mathbb{O})$ with $\operatorname{tr}(p)=1$ and $p \times p=0$. Even better, we can drop the equation $\operatorname{tr}(p)=1$ as long as we promise to work with equivalence classes of nonzero elements satisfying $p \times p=0$, where two such elements are equivalent when one is a nonzero real multiple of the other. Each such equivalence class $[p]$ corresponds to a unique point of $\mathbb{O P}^{2}$, and we get all the points this way.

Given two points $[p]$ and $[q]$, their cross product $p \times q$ is well-defined up to a nonzero real multiple. This suggests that we define a 'line' to be an equivalence class of elements $p \times q \in \mathfrak{h}_{3}(\mathbb{O})^{*}$, where again two such elements are deemed equivalent if one is a nonzero real multiple of the other. Freudenthal showed that we get a projective plane isomorphic to $\mathbb{O P}^{2}$ if we take these as our definitions of points and lines and decree that the point $[p]$ lies on the line $[L]$ if and only if $L(p)=0$. Note that this equation makes sense even though $L$ and $p$ are only well-defined up to nonzero real multiples.

One consequence of all this is that one can recover the structure of $\mathbb{O P}^{2}$ as a projective plane starting from just the determinant on $\mathfrak{h}_{3}(\mathbb{O})$ : we did not need the Jordan algebra structure! However, to get a 'duality' map switching points and lines while preserving the incidence relation, we need a bit more: we need the nondegenerate pairing

$$
\langle a, b\rangle=\operatorname{tr}(a b)
$$


on $\mathfrak{h}_{3}(\mathbb{O})$. This sets up an isomorphism

$$
\mathfrak{h}_{3}(\mathbb{O}) \cong \mathfrak{h}_{3}(\mathbb{O})^{*} .
$$

This isomorphism turns out to map points to lines, and in fact, it sets up a one-toone correspondence between points and lines. We can use this correspondence to think of both points and lines in $\mathbb{O P}^{2}$ as equivalence classes of elements of $\mathfrak{h}_{3}(\mathbb{O})$. In these terms, the point $p$ lies on the line $\ell$ iff $\langle\ell, p\rangle=0$. This relationship is symmetrical! It follows that if we switch points and lines using this correspondence, the incidence relation is preserved.

We thus obtain a very pretty setup for working with $\mathbb{O P}^{2}$. If we use the isomorphism between $\mathfrak{h}_{3}(\mathbb{O})$ and its dual to reinterpret the cross product as a map

$$
\times: \mathfrak{h}_{3}(\mathbb{O}) \times \mathfrak{h}_{3}(\mathbb{O}) \rightarrow \mathfrak{h}_{3}(\mathbb{O}),
$$

then not only is the line through distinct points $[p]$ and $[q]$ given by $[p \times q]$, but also the point in which two distinct lines $[\ell]$ and $[m]$ meet is given by $[\ell \times m]$. A triple of points $[p],[q]$ and $[r]$ is collinear iff $(p, q, r)=0$, and a triple of lines $[\ell]$, $[m],[n]$ meets at a point iff $(\ell, m, n)=0$. In addition, there is a delightful bunch of identities relating the Jordan product, the determinant, the cross product and the inner product in $\mathfrak{h}_{3}(\mathbb{O})$.

For more on octonionic geometry, the reader is urged to consult the original papers by Freudenthal [38, 39], [40, [41], Jacques Tits [93, 94] and Tonny Springer [86], [87, 88. The book by Helmut Salzmann et al. is also good [81. Unfortunately, we must now bid goodbye to this subject and begin our trip through the exceptional groups. However, we shall return to study the symmetries of $\mathbb{O P}^{2}$ and the exceptional Jordan algebra in Sections 4.2 and 4.4 .

\section{Exceptional Lie algebras}

On October 18th, 1887, Wilhelm Killing wrote a letter to Friedrich Engel saying that he had classified the simple Lie algebras. In the next three years, this revolutionary work was published in a series of papers 62. Besides what we now call the 'classical' simple Lie algebras, he claimed to have found 6 'exceptional' ones - new mathematical objects whose existence had never before been suspected. In fact he gave a rigorous construction of only the smallest of these. In his 1894 thesis, Cartan [13] constructed all of them and noticed that the two 52-dimensional exceptional Lie algebras discovered by Killing were isomorphic, so that there are really only 5 .

The Killing-Cartan classification of simple Lie algebras introduced much of the technology that is now covered in any introductory course on the subject, such as roots and weights. In what follows we shall avoid this technology, since we wish instead to see the exceptional Lie algebras as octonionic relatives of the classical ones - slightly eccentric relatives, but still having a close connection to geometry, in particular the Riemannian geometry of projective planes. It is also for this reason that we shall focus on the compact real forms of the simple Lie algebras.

The classical simple Lie algebras can be organized in three infinite families:

$$
\begin{aligned}
& \mathfrak{s o}(n)=\left\{x \in \mathbb{R}[n]: x^{*}=-x, \operatorname{tr}(x)=0\right\}, \\
& \mathfrak{s u}(n)=\left\{x \in \mathbb{C}[n]: x^{*}=-x, \operatorname{tr}(x)=0\right\} \\
& \mathfrak{s p}(n)=\left\{x \in \mathbb{H}[n]: x^{*}=-x\right\} .
\end{aligned}
$$


The corresponding Lie groups are

$$
\begin{aligned}
& \mathrm{SO}(n)=\left\{x \in \mathbb{R}[n]: x x^{*}=1, \operatorname{det}(x)=1\right\}, \\
& \mathrm{SU}(n)=\left\{x \in \mathbb{C}[n]: x x^{*}=1, \operatorname{det}(x)=1\right\}, \\
& \mathrm{Sp}(n)=\left\{x \in \mathbb{H}[n]: x x^{*}=1\right\} .
\end{aligned}
$$

These arise naturally as symmetry groups of projective spaces over $\mathbb{R}, \mathbb{C}$, and $\mathbb{H}$, respectively. More precisely, they arise as groups of isometries: transformations that preserve a specified Riemannian metric. Let us sketch how this works, as a warmup for the exceptional groups.

First consider the projective space $\mathbb{R} \mathbb{P}^{n}$. We can think of this as the unit sphere in $\mathbb{R}^{n+1}$ with antipodal points $x$ and $-x$ identified. It thus inherits a Riemannian metric from the sphere, and the obvious action of the rotation group $\mathrm{O}(n+1)$ as isometries of the sphere yields an action of this group as isometries of $\mathbb{R P}^{n}$ with this metric. In fact, with this metric, the group of all isometries of $\mathbb{R} \mathbb{P}^{n}$ is just

$$
\operatorname{Isom}\left(\mathbb{R P}^{n}\right) \cong \mathrm{O}(n+1) / \mathrm{O}(1)
$$

where $\mathrm{O}(1)=\{ \pm 1\}$ is the subgroup of $\mathrm{O}(n+1)$ that acts trivially on $\mathbb{R P}^{n}$. The Lie algebra of this isometry group is

$$
\mathfrak{i s o m}\left(\mathbb{R} \mathbb{P}^{n}\right) \cong \mathfrak{s o}(n+1) .
$$

The case of $\mathbb{C P}^{n}$ is very similar. We can think of this as the unit sphere in $\mathbb{C}^{n+1}$ with points $x$ and $\alpha x$ identified whenever $\alpha$ is a unit complex number. It thus inherits a Riemannian metric from this sphere, and the unitary group $\mathrm{U}(n+1)$ acts as isometries. If we consider only the connected component of the isometry group and ignore the orientation-reversing isometries coming from complex conjugation, we have

$$
\operatorname{Isom}_{0}\left(\mathbb{C P}^{n}\right) \cong \mathrm{U}(n+1) / \mathrm{U}(1)
$$

where $\mathrm{U}(1)$ is the subgroup that acts trivially on $\mathbb{C P}^{n}$. The Lie algebra of this isometry group is

$$
\mathfrak{i s o m}\left(\mathbb{C P}^{n}\right) \cong \mathfrak{s u}(n+1) .
$$

The case of $\mathbb{H}^{n}$ is subtler, since we must take the noncommutativity of the quaternions into account. We can think of $\mathbb{H} \mathbb{P}^{n}$ as the unit sphere in $\mathbb{H}^{n+1}$ with points $x$ and $\alpha x$ identified whenever $\alpha$ is a unit quaternion, and as before, $\mathbb{H}^{n}$ inherits a Riemannian metric. The group $\operatorname{Sp}(n+1)$ acts as isometries of $\mathbb{H} \mathbb{P}^{n}$, but this action comes from right multiplication, so

$$
\operatorname{Isom}\left(\mathbb{H} \mathbb{P}^{n}\right) \cong \operatorname{Sp}(n+1) /\{ \pm 1\}
$$

since not $\operatorname{Sp}(1)$ but only its center $\{ \pm 1\}$ acts trivially on $\mathbb{H} \mathbb{P}^{n}$ by right multiplication. At the Lie algebra level, this gives

$$
\mathfrak{i s o m}\left(\mathbb{H} \mathbb{P}^{n}\right) \cong \mathfrak{s p}(n+1) .
$$

For lovers of the octonions, it is tempting to try a similar construction starting with $\mathbb{O P}^{2}$. While nonassociativity makes things a bit tricky, we show in Section 4.2 that it can in fact be done. It turns out that $\operatorname{Isom}\left(\mathbb{O P}^{2}\right)$ is one of the exceptional Lie groups, namely $\mathrm{F}_{4}$. Similarly, the exceptional Lie groups $\mathrm{E}_{6}, \mathrm{E}_{7}$ and $\mathrm{E}_{8}$ are in a certain subtle sense the isometry groups of projective planes over the algebras $\mathbb{C} \otimes \mathbb{O}, \mathbb{H} \otimes \mathbb{O}$ and $\mathbb{O} \otimes \mathbb{O}$. Together with $\mathrm{F}_{4}$, these groups can all be defined by the so-called 'magic square' construction, which makes use of much of the algebra we have described so far. We explain three versions of this construction in Section 4.3 
We then treat the groups $\mathrm{E}_{6}, \mathrm{E}_{7}$ and $\mathrm{E}_{8}$ individually in the following sections. But first, we must introduce $\mathrm{G}_{2}$ : the smallest of the exceptional Lie groups, and none other than the automorphism group of the octonions.

4.1. $\mathbf{G}_{2}$. In 1914, Élie Cartan noted that the smallest of the exceptional Lie groups, $\mathrm{G}_{2}$, is the automorphism group of the octonions [14. Its Lie algebra $\mathfrak{g}_{2}$ is therefore $\mathfrak{d} \mathfrak{e r}(\mathbb{O})$, the derivations of the octonions. Let us take these facts as definitions of $\mathrm{G}_{2}$ and its Lie algebra and work out some of the consequences.

What are automorphisms of the octonions like? One way to analyze this involves subalgebras of the octonions. Any octonion $e_{1}$ whose square is -1 generates a subalgebra of $\mathbb{O}$ isomorphic to $\mathbb{C}$. If we then pick any octonion $e_{2}$ with square equal to -1 that anticommutes with $e_{1}$, the elements $e_{1}, e_{2}$ generate a subalgebra isomorphic to $\mathbb{H}$. Finally, if we pick any octonion $e_{3}$ with square equal to -1 that anticommutes with $e_{1}, e_{2}$, and $e_{1} e_{2}$, the elements $e_{1}, e_{2}, e_{3}$ generate all of $\mathbb{O}$. We call such a triple of octonions a basic triple. Given any basic triple, there exists a unique way to define $e_{4}, \ldots, e_{7}$ so that the whole multiplication table in Section 2 holds. In fact, this follows from the remarks on the Cayley-Dickson construction at the end of Section 2.3

It follows that given any two basic triples, there exists a unique automorphism of (1) mapping the first to the second. Conversely, it is obvious that any automorphism maps basic triples to basic triples. This gives a nice description of the group $\mathrm{G}_{2}$, as follows.

Fix a basic triple $e_{1}, e_{2}, e_{3}$. There is a unique automorphism of the octonions mapping this to any other basic triple, say $e_{1}^{\prime}, e_{2}^{\prime}, e_{3}^{\prime}$. Now our description of basic triples so far has been purely algebraic, but we can also view them more geometrically as follows: a basic triple is any triple of unit imaginary octonions (i.e. imaginary octonions of norm one) such that each is orthogonal to the algebra generated by the other two. This means that our automorphism can map $e_{1}$ to any point $e_{1}^{\prime}$ on the 6-sphere of unit imaginary octonions, then map $e_{2}$ to any point $e_{2}^{\prime}$ on the 5 -sphere of unit imaginary octonions that are orthogonal to $e_{1}^{\prime}$, and then map $e_{3}$ to any point $e_{3}^{\prime}$ on the 3 -sphere of unit imaginary octonions that are orthogonal to $e_{1}^{\prime}, e_{2}^{\prime}$ and $e_{1}^{\prime} e_{2}^{\prime}$. It follows that

$$
\operatorname{dim} \mathrm{G}_{2}=\operatorname{dim} S^{6}+\operatorname{dim} S^{5}+\operatorname{dim} S^{3}=14 .
$$

The triality description of the octonions in Section 2.4 gives another picture of $\mathrm{G}_{2}$. First, recall that $\operatorname{Spin}(8)$ is the automorphism group of the triality $t_{8}: V_{8} \times$ $S_{8}^{+} \times S_{8}^{-} \rightarrow \mathbb{R}$. To construct the octonions from this triality we need to pick unit vectors in any two of these spaces, so we can think of $\mathrm{G}_{2}$ as the subgroup of $\operatorname{Spin}(8)$ fixing unit vectors in $V_{8}$ and $S_{8}^{+}$. The subgroup of $\operatorname{Spin}(8)$ fixing a unit vector in $V_{8}$ is just $\operatorname{Spin}(7)$, and when we restrict the representation $S_{8}^{+}$to $\operatorname{Spin}(7)$, we get the spinor representation $S_{7}$. Thus $\mathrm{G}_{2}$ is the subgroup of $\operatorname{Spin}(7)$ fixing a unit vector in $S_{7}$. Since $\operatorname{Spin}(7)$ acts transitively on the unit sphere $S^{7}$ in this spinor representation [2], we have

$$
\operatorname{Spin}(7) / \mathrm{G}_{2}=S^{7} .
$$

It follows that

$$
\operatorname{dim} \mathrm{G}_{2}=\operatorname{dim}(\operatorname{Spin}(7))-\operatorname{dim} S^{7}=21-7=14 .
$$

This picture becomes a bit more vivid if we remember that after choosing unit vectors in $V_{8}$ and $S_{8}^{+}$, we can identify both these representations with the octonions, 
with both unit vectors corresponding to $1 \in \mathbb{O}$. Thus what we are really saying is this: the subgroup of $\operatorname{Spin}(8)$ that fixes 1 in the vector representation on $(\mathbb{O}$ is $\operatorname{Spin}(7)$; the subgroup that fixes 1 in both the vector and right-handed spinor representations is $G_{2}$. This subgroup also fixes the element 1 in the left-handed spinor representation of $\operatorname{Spin}(8)$ on $\mathbb{O}$.

Now, using the vector representation of $\operatorname{Spin}(8)$ on $\mathbb{O}$, we get homomorphisms

$$
\mathrm{G}_{2} \hookrightarrow \operatorname{Spin}(8) \rightarrow \mathrm{SO}(\mathbb{O})
$$

where $\mathrm{SO}(\mathbb{O}) \cong \mathrm{SO}(8)$ is the rotation group of the octonions, viewed as a real vector space with the inner product $\langle x, y\rangle=\operatorname{Re}\left(x^{*} y\right)$. The map from $\operatorname{Spin}(8)$ to $\operatorname{SO}(\mathbb{O})$ is two-to-one, but when we restrict it to $\mathrm{G}_{2}$ we get a one-to-one map

$$
\mathrm{G}_{2} \hookrightarrow \mathrm{SO}(\mathbb{O}) \text {. }
$$

At the Lie algebra level, this construction gives an inclusion

$$
\mathfrak{g}_{2} \hookrightarrow \mathfrak{s o}(\mathbb{O})
$$

where $\mathfrak{s o}(\mathbb{O}) \cong \mathfrak{s o}(8)$ is the Lie algebra of skew-adjoint real-linear transformations of the octonions. Since $\mathfrak{g}_{2}$ is 14 -dimensional and $\mathfrak{s o}(\mathbb{O})$ is 28 -dimensional, it is nice to see exactly where the extra 14 dimensions come from. In fact, they come from two copies of $\operatorname{Im}(\mathbb{O})$, the 7 -dimensional space consisting of all imaginary octonions.

More precisely, we have:

$$
\mathfrak{s o}(\mathbb{O})=\mathfrak{g}_{2} \oplus L_{\operatorname{Im}(\mathbb{O})} \oplus R_{\operatorname{Im}(\mathbb{O})}
$$

(a direct sum of vector spaces, not Lie algebras), where $L_{\operatorname{Im}(\mathbb{O})}$ is the space of linear transformations of $\mathbb{O}$ given by left multiplication by imaginary octonions and $R_{\operatorname{Im}(\mathbb{O})}$ is the space of linear transformations of $\mathbb{O}$ given by right multiplication by imaginary octonions 83]. To see this, we first check that left multiplication by an imaginary octonion is skew-adjoint. Using polarization, it suffices to note that

$$
\langle x, a x\rangle=\operatorname{Re}\left(x^{*}(a x)\right)=\operatorname{Re}\left(\left(x^{*} a\right) x\right)=\operatorname{Re}\left(\left(a^{*} x\right)^{*} x\right)=-\operatorname{Re}\left((a x)^{*} x\right)=-\langle a x, x\rangle
$$

for all $a \in \operatorname{Im}(\mathbb{O})$ and $x \in \mathbb{O}$. Note that this calculation only uses the alternative law, not the associative law, since $x, x^{*}$ and $a$ all lie in the algebra generated by the two elements $a$ and $\operatorname{Im}(x)$. A similar argument shows that right multiplication by an imaginary octonion is skew-adjoint. It follows that $\mathfrak{g}_{2}, L_{\operatorname{Im}(\mathbb{O})}$ and $R_{\operatorname{Im}(\mathbb{O})}$ all naturally lie in $\mathfrak{s o}(8)$. Next, with some easy calculations we can check that

$$
L_{\operatorname{Im}(\mathbb{O})} \cap R_{\operatorname{Im}(\mathbb{O})}=\{0\}
$$

and

$$
\mathfrak{g}_{2} \cap\left(L_{\operatorname{Im}(\mathbb{O})}+R_{\operatorname{Im}(\mathbb{O})}\right)=\{0\} .
$$

Using the fact that the dimensions of the 3 pieces add to 28 , equation (11) follows.

We have seen that $\mathrm{G}_{2}$ sits inside $\mathrm{SO}(8)$, but we can do better: it actually sits inside $\mathrm{SO}(7)$. After all, every automorphism of the octonions fixes the identity and thus preserves the space of octonions orthogonal to the identity. This space is just $\operatorname{Im}(\mathbb{O})$, so we have an inclusion

$$
\mathrm{G}_{2} \hookrightarrow \mathrm{SO}(\operatorname{Im}(\mathbb{O}))
$$

where $\mathrm{SO}(\operatorname{Im}(\mathbb{O})) \cong \mathrm{SO}(7)$ is the rotation group of the imaginary octonions. At the Lie algebra level this gives an inclusion

$$
\mathfrak{g}_{2} \hookrightarrow \mathfrak{s o}(\operatorname{Im}(\mathbb{O})) .
$$


Since $\mathfrak{g}_{2}$ is 14-dimensional and $\mathfrak{s o}(\operatorname{Im}(\mathbb{O}))$ is 21 -dimensional, it is nice to see where the 7 extra dimensions come from. Examining equation (11), it is clear that these extra dimensions must come from the transformations in $L_{\operatorname{Im}(\mathbb{O})} \oplus R_{\operatorname{Im}(\mathbb{O})}$ that annihilate the identity $1 \in \mathbb{O}$. The transformations that do this are precisely those of the form

$$
\operatorname{ad}_{a}=L_{a}-R_{a}
$$

for $a \in \operatorname{Im}(\mathbb{O})$. We thus have

$$
\mathfrak{s o}(\operatorname{Im}(\mathbb{O})) \cong \mathfrak{d e r}(\mathbb{O}) \oplus \operatorname{ad}_{\operatorname{Im}(\mathbb{O})}
$$

where $\operatorname{ad}_{\operatorname{Im}(\mathbb{O})}$ is the 7 -dimensional space of such transformations.

We may summarize some of the above results as follows:

Theorem 4. The compact real form of the Lie algebra $\mathfrak{g}_{2}$ is given by

$$
\mathfrak{g}_{2}=\mathfrak{d e r}(\mathbb{O}) \subset \mathfrak{s o}(\operatorname{Im}(\mathbb{O})) \subset \mathfrak{s o}(\mathbb{O})
$$

and we have

$$
\begin{aligned}
\mathfrak{s o}(\operatorname{Im}(\mathbb{O})) & =\mathfrak{d e r}(\mathbb{O}) \oplus \operatorname{ad}_{\operatorname{Im}(\mathbb{O})} \\
\mathfrak{s o}(\mathbb{O}) & =\mathfrak{d e r}(\mathbb{O}) \oplus L_{\operatorname{Im}(\mathbb{O})} \oplus R_{\operatorname{Im}(\mathbb{O})}
\end{aligned}
$$

where the Lie brackets in $\mathfrak{s o}(\operatorname{Im}(\mathbb{O}))$ and $\mathfrak{s o}(\mathbb{O})$ are built from natural bilinear operations on the summands.

As we have seen, $G_{2}$ has a 7-dimensional representation $\operatorname{Im}(\mathbb{O})$. In fact, this is the smallest nontrivial representation of $\mathrm{G}_{2}$, so it is worth understanding in as many ways as possible. The space $\operatorname{Im}(\mathbb{O})$ has at least three natural structures that are preserved by the transformations in $\mathrm{G}_{2}$. These give more descriptions of $\mathrm{G}_{2}$ as a symmetry group, and they also shed some new light on the octonions. The first two of the structures we describe are analogous to more familiar ones that exist on the 3-dimensional space of imaginary quaternions, $\operatorname{Im}(\mathbb{H})$. The third makes explicit use of the nonassociativity of the octonions.

First, both $\operatorname{Im}(\mathbb{H})$ and $\operatorname{Im}(\mathbb{O})$ are closed under the commutator. In the case of $\operatorname{Im}(\mathbb{H})$, the commutator divided by 2 is the familiar cross product in 3 dimensions:

$$
a \times b=\frac{1}{2}[a, b] .
$$

We can make the same definition for $\operatorname{Im}(\mathbb{O})$, obtaining a 7-dimensional analog of the cross product. For both $\operatorname{Im}(\mathbb{H})$ and $\operatorname{Im}(\mathbb{O})$ the cross product is bilinear and anticommutative. The cross product makes $\operatorname{Im}(\mathbb{H})$ into a Lie algebra, but not $\operatorname{Im}(\mathbb{O})$. For both $\operatorname{Im}(\mathbb{H})$ and $\operatorname{Im}(\mathbb{O})$, the cross product has two nice geometrical properties. On the one hand, its norm is determined by the formula

$$
\|a \times b\|^{2}+\langle a, b\rangle^{2}=\|a\|^{2}\|b\|^{2}
$$

or equivalently,

$$
\|a \times b\|=|\sin \theta|\|a\|\|b\|,
$$

where $\theta$ is the angle between $a$ and $b$. On the other hand, $a \times b$ is orthogonal to $a$ and $b$. Both these properties follow from easy calculations. For $\operatorname{Im}(\mathbb{H})$, these two properties are enough to determine $x \times y$ up to a $\operatorname{sign}$. For $\operatorname{Im}(\mathbb{O})$ they are not but they become so if we also use the fact that $x \times y$ lies inside a copy of $\operatorname{Im}(\mathbb{H})$ that contains $x$ and $y$. 
It is clear that the group of all real-linear transformations of $\operatorname{Im}(\mathbb{H})$ preserving the cross product is just $\mathrm{SO}(3)$, which is also the automorphism group of the quaternions. One can similarly show that the group of real-linear transformations of $\operatorname{Im}(\mathbb{O})$ preserving the cross product is exactly $G_{2}$. To see this, start by noting that any element of $\mathrm{G}_{2}$ preserves the cross product on $\operatorname{Im}(\mathbb{O})$, since the cross product is defined using octonion multiplication. To show that conversely any transformation preserving the cross product lies in $\mathrm{G}_{2}$, it suffices to express the multiplication of imaginary octonions in terms of their cross product. Using this identity:

$$
a \times b=a b+\langle a, b\rangle,
$$

it actually suffices to express the inner product on $\operatorname{Im}(\mathbb{O})$ in terms of the cross product. Here the following identity does the job:

$$
\langle a, b\rangle=-\frac{1}{6} \operatorname{tr}(a \times(b \times \cdot))
$$

where the right-hand side refers to the trace of the map

$$
a \times(b \times \cdot): \operatorname{Im}(\mathbb{O}) \rightarrow \operatorname{Im}(\mathbb{O}) .
$$

Second, both $\operatorname{Im}(\mathbb{H})$ and $\operatorname{Im}(\mathbb{O})$ are equipped with a natural 3-form, or in other words, an alternating trilinear functional. This is given by

$$
\phi(x, y, z)=\langle x, y z\rangle .
$$

In the case of $\operatorname{Im}(\mathbb{H})$ this is just the usual volume form, and the group of reallinear transformations preserving it is $\mathrm{SL}(3, \mathbb{R})$. In the case of $\operatorname{Im}(\mathbb{O})$, the reallinear transformations preserving $\phi$ are exactly those in the group $\mathrm{G}_{2}$. A proof of this by Robert Bryant can be found in Reese Harvey's book [53]. The 3-form $\phi$ is important in the theory of 'Joyce manifolds' [59], which are 7-dimensional Riemannian manifolds with holonomy group equal to $\mathrm{G}_{2}$.

Third, both $\operatorname{Im}(\mathbb{H})$ and $\operatorname{Im}(\mathbb{O})$ are closed under the associator. For $\operatorname{Im}(\mathbb{H})$ this is boring, since the associator vanishes. On the other hand, for $\operatorname{Im}(\mathbb{O})$ the associator is interesting. In fact, it follows from results of Harvey [53] that a real-linear transformation $T: \operatorname{Im}(\mathbb{O}) \rightarrow \operatorname{Im}(\mathbb{O})$ preserves the associator if and only if $\pm T$ lies in $G_{2}$. Thus the symmetry group of the associator is slightly bigger than $G_{2}$ : it is $\mathrm{G}_{2} \times \mathbb{Z}_{2}$.

Now we must make an embarrassing admission: these three structures on $\operatorname{Im}(\mathbb{O})$ are all almost the same thing! Starting with the cross product

$$
\times: \operatorname{Im}(\mathbb{O}) \times \operatorname{Im}(\mathbb{O}) \rightarrow \operatorname{Im}(\mathbb{O})
$$

we can recover the usual inner product on $\operatorname{Im}(\mathbb{O})$ by equation $(13)$. This inner product allows us to dualize the cross product and obtain a trilinear functional, which is, up to a constant, just the 3 -form

$$
\phi: \operatorname{Im}(\mathbb{O}) \times \operatorname{Im}(\mathbb{O}) \times \operatorname{Im}(\mathbb{O}) \rightarrow \mathbb{R} .
$$

The cross product also determines an orientation on $\operatorname{Im}(\mathbb{O})$ (we leave this as an exercise for the reader). This allows us to take the Hodge dual of $\phi$, obtaining a 4 -form $\psi$, i.e. an alternating tetralinear functional

$$
\psi: \operatorname{Im}(\mathbb{O}) \times \operatorname{Im}(\mathbb{O}) \times \operatorname{Im}(\mathbb{O}) \times \operatorname{Im}(\mathbb{O}) \rightarrow \mathbb{R} .
$$

Dualizing yet again, this gives a ternary operation which, up to a constant multiple, is the associator:

$$
[\cdot, \cdot, \cdot]: \operatorname{Im}(\mathbb{O}) \times \operatorname{Im}(\mathbb{O}) \times \operatorname{Im}(\mathbb{O}) \rightarrow \operatorname{Im}(\mathbb{O})
$$


We conclude this section with a handy explicit formula for all the derivations of the octonions. In an associative algebra $A$, any element $x$ defines an inner derivation $\operatorname{ad}_{x}: A \rightarrow A$ by

$$
\operatorname{ad}_{x}(a)=[x, a]
$$

where the bracket denotes the commutator $x a-a x$. In a nonassociative algebra, this formula usually does not define a derivation. However, if $A$ is alternative, any pair of elements $x, y \in A$ define a derivation $D_{x, y}: A \rightarrow A$ by

$$
D_{x, y} a=[[x, y], a]-3[x, y, a]
$$

where $[a, b, x]$ denotes the associator $(a b) x-a(b x)$. Moreover, when $A$ is a normed division algebra, every derivation is a linear combination of derivations of this form. Unfortunately, proving these facts seems to require some brutal calculations 83 .

4.2. $\mathbf{F}_{4}$. The second smallest of the exceptional Lie groups is the 52-dimensional group $\mathrm{F}_{4}$. The geometric meaning of this group became clear in a number of nearly simultaneous papers by various mathematicians. In 1949, Jordan constructed the octonionic projective plane using projections in $\mathfrak{h}_{3}(\mathbb{O})$. One year later, Armand Borel [10] noted that $\mathrm{F}_{4}$ is the isometry group of a 16-dimensional projective plane. In fact, this plane is none other than $\mathbb{O P}^{2}$. Also in 1950 , Claude Chevalley and Richard Schafer [20] showed that $\mathrm{F}_{4}$ is the automorphism group of $\mathfrak{h}_{3}(\mathbb{O})$. In 1951, Freudenthal [38] embarked upon a long series of papers in which he described not only $\mathrm{F}_{4}$ but also the other exceptional Lie groups using octonionic projective geometry. To survey these developments, one still cannot do better than to read his classic 1964 paper on Lie groups and the foundations of geometry [41].

Let us take Chevalley and Schafer's result as the definition of $\mathrm{F}_{4}$ :

$$
\mathrm{F}_{4}=\operatorname{Aut}\left(\mathfrak{h}_{3}(\mathbb{O})\right) .
$$

Its Lie algebra is thus

$$
\mathfrak{f}_{4}=\mathfrak{d e r}\left(\mathfrak{h}_{3}(\mathbb{O})\right) .
$$

As we saw in Section 3.4 points of $\mathbb{O P}^{2}$ correspond to trace-1 projections in the exceptional Jordan algebra. It follows that $\mathrm{F}_{4}$ acts as transformations of $\mathbb{O P}^{2}$. In fact, we can equip $\mathbb{O P}^{2}$ with a Riemannian metric for which $\mathrm{F}_{4}$ is the isometry group. To get a sense of how this works, let us describe $\mathbb{O P}^{2}$ as a quotient space of $\mathrm{F}_{4}$.

In Section 3.4 we saw that the exceptional Jordan algebra can be built using natural operations on the scalar, vector and spinor representations of $\operatorname{Spin}(9)$. This implies that $\operatorname{Spin}(9)$ is a subgroup of $\mathrm{F}_{4}$. Equation (9) makes it clear that $\operatorname{Spin}(9)$ is precisely the subgroup fixing the element

$$
\left(\begin{array}{lll}
1 & 0 & 0 \\
0 & 0 & 0 \\
0 & 0 & 0
\end{array}\right) .
$$

Since this element is a trace-one projection, it corresponds to a point of $\mathbb{O P}^{2}$. We have already seen that $\mathrm{F}_{4}$ acts transitively on $\mathbb{O P}^{2}$. It follows that

$$
\mathbb{O P}^{2} \cong \mathrm{F}_{4} / \operatorname{Spin}(9) \text {. }
$$


This fact has various nice spinoffs. First, it gives an easy way to compute the dimension of $\mathrm{F}_{4}$ :

$$
\operatorname{dim}\left(\mathrm{F}_{4}\right)=\operatorname{dim}(\operatorname{Spin}(9))+\operatorname{dim}\left(\mathbb{O P}^{2}\right)=36+16=52 .
$$

Second, since $\mathrm{F}_{4}$ is compact, we can take any Riemannian metric on $\mathbb{O P}^{2}$ and average it with respect to the action of this group. The isometry group of the resulting metric will automatically include $\mathrm{F}_{4}$ as a subgroup. With more work [6], one can show that actually

$$
\mathrm{F}_{4}=\operatorname{Isom}\left(\mathbb{O P}^{2}\right)
$$

and thus

$$
\mathfrak{f}_{4}=\mathfrak{i s o m}\left(\mathbb{O P} \mathbb{P}^{2}\right)
$$

Equation (15) also implies that the tangent space of our chosen point in $\mathbb{O P}^{2}$ is isomorphic to $\mathfrak{f}_{4} / \mathfrak{s o}(9)$. But we already know that this tangent space is just $\mathbb{O}^{2}$, or in other words, the spinor representation of $\mathfrak{s o}(9)$. We thus have

$$
\mathfrak{f}_{4} \cong \mathfrak{s o}(9) \oplus S_{9}
$$

as vector spaces, where $\mathfrak{s o}(9)$ is a Lie subalgebra. The bracket in $\mathfrak{f}_{4}$ is built from the bracket in $\mathfrak{s o}(9)$, the action $\mathfrak{s o}(9) \otimes S_{9} \rightarrow S_{9}$, and the map $S_{9} \otimes S_{9} \rightarrow \mathfrak{s o}(9)$ obtained by dualizing this action. We can also rewrite this description of $\mathfrak{f}_{4}$ in terms of the octonions, as follows:

$$
\mathfrak{f}_{4} \cong \mathfrak{s o}(\mathbb{O} \oplus \mathbb{R}) \oplus \mathbb{O}^{2}
$$

This last formula suggests that we decompose $\mathfrak{f}_{4}$ further using the splitting of $\mathbb{O} \oplus \mathbb{R}$ into $\mathbb{O}$ and $\mathbb{R}$. It is easily seen by looking at matrices that for all $n, m$ we have

$$
\mathfrak{s o}(n+m) \cong \mathfrak{s o}(n) \oplus \mathfrak{s o}(m) \oplus V_{n} \otimes V_{m}
$$

Moreover, when we restrict the representation $S_{9}$ to $\mathfrak{s o}(8)$, it splits as a direct sum $S_{8}^{+} \oplus S_{8}^{-}$. Using these facts and equation (16), we see

$$
\mathfrak{f}_{4} \cong \mathfrak{s o}(8) \oplus V_{8} \oplus S_{8}^{+} \oplus S_{8}^{-}
$$

This formula emphasizes the close relation between $\mathfrak{f}_{4}$ and triality: the Lie bracket in $\mathfrak{f}_{4}$ is completely built out of maps involving $\mathfrak{s o}(8)$ and its three 8 -dimensional irreducible representations! We can rewrite this in a way that brings out the role of the octonions:

$$
\mathfrak{f}_{4} \cong \mathfrak{s o}(\mathbb{O}) \oplus \mathbb{O}^{3} \text {. }
$$

While elegant, none of these descriptions of $\mathfrak{f}_{4}$ gives a convenient picture of all the derivations of the exceptional Jordan algebra. In fact, there is a nice picture of this sort for $\mathfrak{h}_{3}(\mathbb{K})$ whenever $\mathbb{K}$ is a normed division algebra. One way to get a derivation of the Jordan algebra $\mathfrak{h}_{3}(\mathbb{K})$ is to take a derivation of $\mathbb{K}$ and let it act on each entry of the matrices in $\mathfrak{h}_{3}(\mathbb{K})$. Another way uses elements of

$$
\mathfrak{s a}_{3}(\mathbb{K})=\left\{x \in \mathbb{K}[3]: x^{*}=-x, \operatorname{tr}(x)=0\right\} .
$$

Given $x \in \mathfrak{s a}_{3}(\mathbb{K})$, there is a derivation $\operatorname{ad}_{x}$ of $\mathfrak{h}_{3}(\mathbb{K})$ given by

$$
\operatorname{ad}_{x}(a)=[x, a] .
$$


In fact [5], every derivation of $\mathfrak{h}_{3}(\mathbb{K})$ can be uniquely expressed as a linear combination of derivations of these two sorts, so we have

$$
\mathfrak{d e r}\left(\mathfrak{h}_{3}(\mathbb{K})\right) \cong \mathfrak{d e r}(\mathbb{K}) \oplus \mathfrak{s a} \mathfrak{a}_{3}(\mathbb{K})
$$

as vector spaces. In the case of the octonions, this decomposition says that

$$
\mathfrak{f}_{4} \cong \mathfrak{g}_{2} \oplus \mathfrak{s a}_{3}(\mathbb{O}) \text {. }
$$

In equation (19), the subspace $\mathfrak{d e r}(\mathbb{K})$ is always a Lie subalgebra, but $\mathfrak{s a} \mathfrak{a}_{3}(\mathbb{K})$ is not unless $\mathbb{K}$ is commutative and associative - in which case $\mathfrak{d e r}(\mathbb{K})$ vanishes. Nonetheless, there is a formula for the brackets in $\mathfrak{d e r}\left(\mathfrak{h}_{3}(\mathbb{K})\right)$ which applies in every case 75]. Given $D, D^{\prime} \in \mathfrak{d e r}(\mathbb{K})$ and $x, y \in \mathfrak{s a}_{3}(\mathbb{K})$, we have

$$
\begin{aligned}
& {\left[D, D^{\prime}\right]=D D^{\prime}-D^{\prime} D} \\
& {\left[D, \operatorname{ad}_{x}\right]=\operatorname{ad}_{D x}} \\
& {\left[\operatorname{ad}_{x}, \operatorname{ad}_{y}\right]=\operatorname{ad}_{[x, y]_{0}}+\frac{1}{3} \sum_{i, j=1}^{3} D_{x_{i j}, y_{i j}}}
\end{aligned}
$$

where $D$ acts on $x$ componentwise, $[x, y]_{0}$ is the trace-free part of the commutator $[x, y]$, and $D_{x_{i j}, y_{i j}}$ is the derivation of $\mathbb{K}$ defined using equation (14).

Summarizing these different descriptions of $\mathfrak{f}_{4}$, we have:

Theorem 5. The compact real form of $\mathfrak{f}_{4}$ is given by

$$
\begin{aligned}
\mathfrak{f}_{4} & \cong \mathfrak{i s o m}\left(\mathbb{O P} \mathbb{P}^{2}\right) \\
& \cong \mathfrak{d e r}\left(\mathfrak{h}_{3}(\mathbb{O})\right) \\
& \cong \mathfrak{d e r}(\mathbb{O}) \oplus \mathfrak{s a} \mathfrak{a}_{3}(\mathbb{O}) \\
& \cong \mathfrak{s o}(\mathbb{O} \oplus \mathbb{R}) \oplus \mathbb{O}^{2} \\
& \cong \mathfrak{s o}(\mathbb{O}) \oplus \mathbb{O}^{3}
\end{aligned}
$$

where in each case the Lie bracket is built from natural bilinear operations on the summands.

4.3. The magic square. Around 1956, Boris Rosenfeld [78] had the remarkable idea that just as $\mathrm{F}_{4}$ is the isometry group of the projective plane over the octonions, the exceptional Lie groups $\mathrm{E}_{6}, \mathrm{E}_{7}$ and $\mathrm{E}_{8}$ are the isometry groups of projective planes over the following three algebras, respectively:

- the bioctonions, $\mathbb{C} \otimes \mathbb{O}$,

- the quateroctonions, $\mathbb{H} \otimes \mathbb{O}$,

- the octooctonions, $\mathbb{O} \otimes \mathbb{O}$.

There is definitely something right about this idea, because one would expect these projective planes to have dimensions 32,64 , and 128, and there indeed do exist compact Riemannian manifolds with these dimensions having $\mathrm{E}_{6}, \mathrm{E}_{7}$ and $\mathrm{E}_{8}$ as their isometry groups. The problem is that the bioctonions, quateroctonions and and octooctonions are not division algebras, so it is a nontrivial matter to define projective planes over them!

The situation is not so bad for the bioctonions: $\mathfrak{h}_{3}(\mathbb{C} \otimes \mathbb{O})$ is a simple Jordan algebra, though not a formally real one, and one can use this to define $(\mathbb{C} \otimes \mathbb{O}) \mathbb{P}^{2}$ in a manner modeled after one of the constructions of $\mathbb{O P}^{2}$. Rosenfeld claimed that a similar construction worked for the quateroctonions and octooctonions, but this appears to be false. Among other problems, $\mathfrak{h}_{3}(\mathbb{H} \otimes \mathbb{O})$ and $\mathfrak{h}_{3}(\mathbb{O} \otimes \mathbb{O})$ do not become Jordan algebras under the product $a \circ b=\frac{1}{2}(a b+b a)$. Scattered throughout the 
literature 6], 41, 42] one can find frustrated comments about the lack of a really nice construction of $(\mathbb{H} \otimes \mathbb{O}) \mathbb{P}^{2}$ and $(\mathbb{O} \otimes \mathbb{O}) \mathbb{P}^{2}$. One problem is that these spaces do not satisfy the usual axioms for a projective plane. Tits addressed this problem in his theory of 'buildings', which allows one to construct a geometry having any desired algebraic group as symmetries [96]. But alas, it still seems that the quickest way to get our hands on the quateroctonionic and octooctonionic 'projective planes' is by starting with the Lie groups $\mathrm{E}_{7}$ and $\mathrm{E}_{8}$ and then taking quotients by suitable subgroups.

In short, more work must be done before we can claim to fully understand the geometrical meaning of the Lie groups $\mathrm{E}_{6}, \mathrm{E}_{7}$ and $\mathrm{E}_{8}$. Luckily, Rosenfeld's ideas can be used to motivate a nice construction of their Lie algebras. This goes by the name of the 'magic square'. Tits 95] and Freudenthal 40 found two very different versions of this construction in about 1958, but we shall start by presenting a simplified version published by E. B. Vinberg 98 in 1966.

First consider the projective plane $\mathbb{K} \mathbb{P}^{2}$ when $\mathbb{K}$ is a normed division algebra $\mathbb{K}$. The points of this plane are the rank- 1 projections in the Jordan algebra $\mathfrak{h}_{3}(\mathbb{K})$, and this plane admits a Riemannian metric such that

$$
\mathfrak{i s o m}\left(\mathbb{K} \mathbb{P}^{2}\right) \cong \mathfrak{d e r}\left(\mathfrak{h}_{3}(\mathbb{K})\right) .
$$

Moreover, we have seen in equation (19) that

$$
\mathfrak{d e r}\left(\mathfrak{h}_{3}(\mathbb{K})\right) \cong \mathfrak{d e r}(\mathbb{K}) \oplus \mathfrak{s a}_{3}(\mathbb{K}) .
$$

Combined with Rosenfeld's observations, these facts might lead one to hope that whenever we have a pair of normed division algebras $\mathbb{K}$ and $\mathbb{K}^{\prime}$, there is a Riemannian manifold $\left(\mathbb{K} \otimes \mathbb{K}^{\prime}\right) \mathbb{P}^{2}$ with

$$
\mathfrak{i s o m}\left(\left(\mathbb{K} \otimes \mathbb{K}^{\prime}\right) \mathbb{P}^{2}\right) \cong \mathfrak{d e r}(\mathbb{K}) \oplus \mathfrak{d e r}\left(\mathbb{K}^{\prime}\right) \oplus \mathfrak{s a} \mathfrak{a}_{3}\left(\mathbb{K} \otimes \mathbb{K}^{\prime}\right)
$$

where for any $*$-algebra $A$ we define

$$
\begin{aligned}
& \mathfrak{s h}_{n}(A)=\left\{x \in A[n]: x^{*}=x, \operatorname{tr}(x)=0\right\} \\
& \mathfrak{s a}_{n}(A)=\left\{x \in A[n]: x^{*}=-x, \operatorname{tr}(x)=0\right\} .
\end{aligned}
$$

This motivated Vinberg's definition of the magic square Lie algebras:

$$
\mathrm{M}\left(\mathbb{K}, \mathbb{K}^{\prime}\right)=\mathfrak{d} \mathfrak{e r}(\mathbb{K}) \oplus \mathfrak{d e r}\left(\mathbb{K}^{\prime}\right) \oplus \mathfrak{s a} \mathfrak{a}_{3}\left(\mathbb{K} \otimes \mathbb{K}^{\prime}\right) .
$$

Now, when $\mathbb{K} \otimes \mathbb{K}^{\prime}$ is commutative and associative, $\mathfrak{s a}_{3}\left(\mathbb{K} \otimes \mathbb{K}^{\prime}\right)$ is a Lie algebra with the commutator as its Lie bracket, but in the really interesting cases it is not. Thus to make $\mathrm{M}\left(\mathbb{K}, \mathbb{K}^{\prime}\right)$ into a Lie algebra we must give it a rather subtle bracket. We have already seen the special case $\mathbb{K}^{\prime}=\mathbb{R}$ in equation (20). In general, the Lie bracket in $\mathrm{M}\left(\mathbb{K}, \mathbb{K}^{\prime}\right)$ is given as follows:

1. $\mathfrak{d e r}(\mathbb{K})$ and $\mathfrak{d e r}\left(\mathbb{K}^{\prime}\right)$ are commuting Lie subalgebras of $\mathrm{M}\left(\mathbb{K}, \mathbb{K}^{\prime}\right)$.

2. The bracket of $D \in \mathfrak{d e r}(K) \oplus \mathfrak{d e r}\left(\mathbb{K}^{\prime}\right)$ with $x \in \mathfrak{s a}_{3}\left(\mathbb{K} \otimes \mathbb{K}^{\prime}\right)$ is given by applying $D$ to every entry of the matrix $x$, using the natural action of $\mathfrak{d e r}(K) \oplus \mathfrak{d e r}\left(\mathbb{K}^{\prime}\right)$ as derivations of $\mathbb{K} \otimes \mathbb{K}^{\prime}$.

3. Given $X, Y \in \mathfrak{s a}_{3}\left(\mathbb{K} \otimes \mathbb{K}^{\prime}\right)$,

$$
[X, Y]=[X, Y]_{0}+\frac{1}{3} \sum_{i, j=1}^{3} D_{X_{i j}, Y_{i j}} .
$$


Here $[X, Y]_{0}$ is the traceless part of the $3 \times 3$ matrix $[X, Y]$, and given $x, y \in$ $\mathbb{K} \otimes \mathbb{K}^{\prime}$ we define $D_{x, y} \in \mathfrak{d e r}(\mathbb{K}) \oplus \mathfrak{d e r}\left(\mathbb{K}^{\prime}\right)$ in the following way: $D_{x, y}$ is realbilinear in $x$ and $y$, and

$$
D_{a \otimes a^{\prime}, b \otimes b^{\prime}}=\left\langle a^{\prime}, b^{\prime}\right\rangle D_{a, b}+\langle a, b\rangle D_{a^{\prime}, b^{\prime}}
$$

where $a, b \in \mathbb{K}, a^{\prime}, b^{\prime} \in \mathbb{K}^{\prime}$, and $D_{a, b}, D_{a^{\prime}, b^{\prime}}$ are defined as in equation (14).

With this construction we magically obtain the following square of Lie algebras:

\begin{tabular}{|l|c|c|c|c|}
\hline & $\mathbb{K}^{\prime}=\mathbb{R}$ & $\mathbb{K}^{\prime}=\mathbb{C}$ & $\mathbb{K}^{\prime}=\mathbb{H}$ & $\mathbb{K}^{\prime}=\mathbb{O}$ \\
\hline $\mathbb{K}=\mathbb{R}$ & $\mathfrak{s}(3)$ & $\mathfrak{s u}(3)$ & $\mathfrak{s p}(3)$ & $\mathfrak{f}_{4}$ \\
\hline $\mathbb{K}=\mathbb{C}$ & $\mathfrak{s u}(3)$ & $\mathfrak{s u}(3) \oplus \mathfrak{s u}(3)$ & $\mathfrak{s u}(6)$ & $\mathfrak{e}_{6}$ \\
\hline $\mathbb{K}=\mathbb{H}$ & $\mathfrak{s p}(3)$ & $\mathfrak{s u}(6)$ & $\mathfrak{s o}(12)$ & $\mathfrak{e}_{7}$ \\
\hline $\mathbb{K}=\mathbb{O}$ & $\mathfrak{f}_{4}$ & $\mathfrak{e}_{6}$ & $\mathfrak{e}_{7}$ & $\mathfrak{e}_{8}$ \\
\hline
\end{tabular}

TABle 5. Magic Square Lie Algebras $\mathrm{M}\left(\mathbb{K}, \mathbb{K}^{\prime}\right)$

We will mainly be interested in the last row (or column), which is the one involving the octonions. In this case we can take the magic square construction as defining the Lie algebras $\mathfrak{f}_{4}, \mathfrak{e}_{6}, \mathfrak{e}_{7}$ and $\mathfrak{e}_{8}$. This definition turns out to be consistent with our earlier definition of $\mathfrak{f}_{4}$.

Starting from Vinberg's definition of the magic square Lie algebras, we can easily recover Tits' original definition. To do so, we need two facts. First,

$$
\mathfrak{s a}_{3}\left(\mathbb{K} \otimes \mathbb{K}^{\prime}\right) \cong \mathfrak{s a}_{3}\left(\mathbb{K}^{\prime}\right) \oplus\left(\operatorname{Im}(\mathbb{K}) \otimes \mathfrak{s h}_{3}\left(\mathbb{K}^{\prime}\right)\right)
$$

This is easily seen by direct examination of the relevant matrices. Second,

$$
\mathfrak{d e r}\left(\mathfrak{h}_{3}(\mathbb{K})\right) \cong \mathfrak{d e r}(\mathbb{K}) \oplus \mathfrak{s a} \mathfrak{a}_{3}(\mathbb{K})
$$

as vector spaces. This is just equation (19). Starting with Vinberg's definition and applying these two facts, we obtain

$$
\begin{aligned}
& \mathrm{M}\left(\mathbb{K}, \mathbb{K}^{\prime}\right)=\operatorname{der}(\mathbb{K}) \oplus \mathfrak{d e r}\left(\mathbb{K}^{\prime}\right) \oplus \mathfrak{s a} \mathfrak{a}_{3}\left(\mathbb{K} \otimes \mathbb{K}^{\prime}\right) \\
& \left.\cong \mathfrak{d e r}(\mathbb{K}) \oplus \mathfrak{d e r}\left(\mathbb{K}^{\prime}\right) \oplus \mathfrak{s a} \mathfrak{a}_{3}\left(\mathbb{K}^{\prime}\right) \oplus(\operatorname{Im}(\mathbb{K}) \otimes \mathfrak{s h})_{3}\left(\mathbb{K}^{\prime}\right)\right) \\
& \cong \operatorname{der}(\mathbb{K}) \oplus \mathfrak{d e r}\left(\mathfrak{h}_{3}\left(\mathbb{K}^{\prime}\right)\right) \oplus\left(\operatorname{Im}(\mathbb{K}) \otimes \mathfrak{s h}_{3}\left(\mathbb{K}^{\prime}\right)\right) \text {. }
\end{aligned}
$$

The last line is Tits' definition of the magic square Lie algebras. Unlike Vinberg's, it is not manifestly symmetrical in $\mathbb{K}$ and $\mathbb{K}^{\prime}$. This unhappy feature is somewhat made up for by the fact that $\mathfrak{d e r}(\mathbb{K}) \oplus \mathfrak{d e r}\left(\mathfrak{h}_{3}\left(\mathbb{K}^{\prime}\right)\right)$ is a nice big Lie subalgebra. This subalgebra acts on $\operatorname{Im}(\mathbb{K}) \otimes \mathfrak{s h}_{3}\left(\mathbb{K}^{\prime}\right)$ in an obvious way, using the fact that any derivation of $\mathbb{K}$ maps $\operatorname{Im}(\mathbb{K})$ to itself, and any derivation of $\mathfrak{h}_{3}\left(\mathbb{K}^{\prime}\right)$ maps $\mathfrak{s h}_{3}\left(\mathbb{K}^{\prime}\right)$ to itself. However, the bracket of two elements of $\left(\operatorname{Im}(\mathbb{K}) \otimes \mathfrak{s h}_{3}\left(\mathbb{K}^{\prime}\right)\right)$ is a bit of a mess.

Yet another description of the magic square was recently given by Barton and Sudbery [5]. This one emphasizes the role of trialities. Let $\mathfrak{t r i}(\mathbb{K})$ be the Lie algebra of the group Aut $(t)$, where $t$ is the normed triality giving the normed division algebra $\mathbb{K}$. From equation (5) we have

$$
\begin{aligned}
& \mathfrak{t r i}(\mathbb{R}) \cong\{0\} \\
& \mathfrak{t r i}(\mathbb{C}) \cong \mathfrak{u}(1)^{2} \\
& \mathfrak{t r i}(\mathbb{H}) \cong \mathfrak{s}(1)^{3} \\
& \mathfrak{t r i}(\mathbb{O}) \cong \mathfrak{s o}(8)
\end{aligned}
$$


To express the magic square in terms of these Lie algebras, we need three facts. First, it is easy to see that

$$
\mathfrak{s h}_{3}(\mathbb{K}) \cong \mathbb{K}^{3} \oplus \mathbb{R}^{2}
$$

Second, Barton and Sudbery show that as vector spaces,

$$
\mathfrak{d e r}\left(\mathfrak{h}_{3}(\mathbb{K})\right) \cong \mathfrak{t r i}(\mathbb{K}) \oplus \mathbb{K}^{3} .
$$

This follows in a case-by-case way from equation (21), but they give a unified proof that covers all cases. Third, they show that as vector spaces,

$$
\mathfrak{t r i}(\mathbb{K}) \cong \mathfrak{d} \mathfrak{e r}(\mathbb{K}) \oplus \operatorname{Im}(\mathbb{K})^{2} .
$$

Now starting with Tits' definition of the magic square, applying the first two facts, regrouping terms, and applying the third fact, we obtain Barton and Sudbery's version of the magic square:

$$
\begin{aligned}
\mathrm{M}\left(\mathbb{K}, \mathbb{K}^{\prime}\right) & \cong \mathfrak{d e r}(\mathbb{K}) \oplus \mathfrak{d e r}\left(\mathfrak{h}_{3}\left(\mathbb{K}^{\prime}\right)\right) \oplus\left(\operatorname{Im}(\mathbb{K}) \otimes \mathfrak{s h}_{3}\left(\mathbb{K}^{\prime}\right)\right) \\
& \cong \mathfrak{d e r}(\mathbb{K}) \oplus \mathfrak{t r i}\left(\mathbb{K}^{\prime}\right) \oplus \mathbb{K}^{\prime 3} \oplus \operatorname{Im}(\mathbb{K}) \otimes\left(\mathbb{K}^{\prime 3} \oplus \mathbb{R}^{2}\right) \\
& \cong \mathfrak{d e r}(\mathbb{K}) \oplus \operatorname{Im}(\mathbb{K})^{2} \oplus \mathfrak{t r i}\left(\mathbb{K}^{\prime}\right) \oplus\left(\mathbb{K} \otimes \mathbb{K}^{\prime}\right)^{3} \\
& \cong \mathfrak{t r i}(\mathbb{K}) \oplus \mathfrak{t r i}\left(\mathbb{K}^{\prime}\right) \oplus\left(\mathbb{K} \otimes \mathbb{K}^{\prime}\right)^{3}
\end{aligned}
$$

In the next three sections we use all these different versions of the magic square to give lots of octonionic descriptions of $\mathfrak{e}_{6}, \mathfrak{e}_{7}$ and $\mathfrak{e}_{8}$. To save space, we usually omit the formulas for the Lie bracket in these descriptions. However, the patient reader can reconstruct these with the help of Barton and Sudbery's paper, which is packed with useful formulas.

As we continue our tour through the exceptional Lie algebras, we shall make contact with Adams' work 2] constructing $\mathfrak{f}_{4}, \mathfrak{e}_{6}, \mathfrak{e}_{7}$, and $\mathfrak{e}_{8}$ with the help of spinors and rotation group Lie algebras:

$$
\begin{aligned}
\mathfrak{f}_{4} \cong \mathfrak{s o}(9) \oplus S_{9} \\
\mathfrak{e}_{6} \cong \mathfrak{s o}(10) \oplus \mathfrak{u}(1) \oplus S_{10} \\
\mathfrak{e}_{7} \cong \mathfrak{s o}(12) \oplus \mathfrak{s p}(1) \oplus S_{12}^{+} \\
\mathfrak{e}_{8} \cong \mathfrak{s o}(16) \oplus S_{16}^{+}
\end{aligned}
$$

as vector spaces. Note that the numbers $9,10,12$ and 16 are 8 more than the dimensions of $\mathbb{R}, \mathbb{C}, \mathbb{H}$ and $\mathbb{O}$. As usual, this is no coincidence! In terms of the octonions, Bott periodicity implies that

$$
S_{n+8} \cong S_{n} \otimes \mathbb{O}^{2} \text {. }
$$

This gives the following description of spinors in dimensions $\leq 16$ :

\begin{tabular}{|l|l|}
\hline$S_{1}=\mathbb{R}$ & $S_{9}=\mathbb{O}^{2}$ \\
\hline$S_{2}=\mathbb{C}$ & $S_{10}=(\mathbb{C} \otimes \mathbb{O})^{2}$ \\
\hline$S_{3}=\mathbb{H}$ & $S_{11}=(\mathbb{H} \otimes \mathbb{O})^{2}$ \\
\hline$S_{4}^{ \pm}=\mathbb{H}$ & $S_{12}^{ \pm}=(\mathbb{H} \otimes \mathbb{O})^{2}$ \\
\hline$S_{5}=\mathbb{H}^{2}$ & $S_{13}=\left(\mathbb{H}^{2} \otimes \mathbb{O}\right)^{2}$ \\
\hline$S_{6}=\mathbb{C}^{4}$ & $S_{14}=\left(\mathbb{C}^{4} \otimes \mathbb{O}\right)^{2}$ \\
\hline$S_{7}=\mathbb{O}$ & $S_{15}=(\mathbb{O} \otimes \mathbb{O})^{2}$ \\
\hline$S_{8}^{ \pm}=\mathbb{O}$ & $S_{16}^{ \pm}=(\mathbb{O} \otimes \mathbb{O})^{2}$ \\
\hline
\end{tabular}

TABLE 6. Spinor Representations Revisited 
Since spinors in dimensions $1,2,4$ and 8 are isomorphic to the division algebras $\mathbb{R}, \mathbb{C}, \mathbb{H}$ and $\mathbb{O}$, spinors in dimensions 8 higher are isomorphic to the 'planes' $\mathbb{O}^{2}$, $(\mathbb{C} \otimes \mathbb{O})^{2},(\mathbb{H} \otimes \mathbb{O})^{2}$ and $(\mathbb{O} \otimes \mathbb{O})^{2}$ - and are thus closely linked to $\mathfrak{f}_{4}, \mathfrak{e}_{6}, \mathfrak{e}_{7}$ and $\mathfrak{e}_{8}$, thanks to the magic square.

4.4. $\mathbf{E}_{6}$. We begin with the 78-dimensional exceptional Lie group $\mathrm{E}_{6}$. As we mentioned in Section 3.4, there is a nice description of a certain noncompact real form of $\mathrm{E}_{6}$ as the group of collineations of $\mathbb{O P}^{2}$, or equivalently, the group of determinantpreserving linear transformations of $\mathfrak{h}_{3}(\mathbb{O})$. But before going into these, we consider the magic square constructions of the Lie algebra $\mathfrak{e}_{6}$. Vinberg's construction gives

$$
\mathfrak{e}_{6}=\mathfrak{d e r}(\mathbb{O}) \oplus \mathfrak{s a} \mathfrak{a}_{3}(\mathbb{C} \otimes \mathbb{O}) .
$$

Tits' construction, which is asymmetrical, gives

$$
\mathfrak{e}_{6} \cong \mathfrak{d e r}\left(\mathfrak{h}_{3}(\mathbb{O})\right) \oplus \mathfrak{s h}_{3}(\mathbb{O})
$$

and also

$$
\mathfrak{e}_{6} \cong \mathfrak{d e r}(\mathbb{O}) \oplus \mathfrak{d e r}\left(\mathfrak{h}_{3}(\mathbb{C})\right) \oplus\left(\operatorname{Im}(\mathbb{O}) \otimes \mathfrak{s h} \mathfrak{h}_{3}(\mathbb{C})\right) .
$$

The Barton-Sudbery construction gives

$$
\mathfrak{e}_{6} \cong \operatorname{tri}(\mathbb{O}) \oplus \mathfrak{t r i}(\mathbb{C}) \oplus(\mathbb{C} \otimes \mathbb{O})^{3} .
$$

We can use any of these to determine the dimension of $\mathfrak{e}_{6}$. For example, we have

$$
\operatorname{dim}\left(\mathfrak{e}_{6}\right)=\operatorname{dim}\left(\mathfrak{d e r}\left(\mathfrak{h}_{3}(\mathbb{O})\right)\right)+\operatorname{dim}\left(\mathfrak{s h}_{3}(\mathbb{O})\right)=52+26=78 .
$$

Starting from the Barton-Sudbery construction and using the concrete descriptions of $\mathfrak{t r i}(\mathbb{O})$ and $\mathfrak{t r i}(\mathbb{C})$ from equation (21), we obtain

$$
\mathfrak{e}_{6} \cong \mathfrak{s o}(\mathbb{O}) \oplus \mathfrak{s o}(\mathbb{C}) \oplus \operatorname{Im}(\mathbb{C}) \oplus(\mathbb{C} \otimes \mathbb{O})^{3} .
$$

Using equation (17), we may rewrite this as

$$
\mathfrak{e}_{6} \cong \mathfrak{s o}(\mathbb{O} \oplus \mathbb{C}) \oplus \operatorname{Im}(\mathbb{C}) \oplus(\mathbb{C} \otimes \mathbb{O})^{2},
$$

and it turns out that the summand $\mathfrak{s o}(\mathbb{O} \oplus \mathbb{C}) \oplus \operatorname{Im}(\mathbb{C})$ is actually a Lie subalgebra of $\mathfrak{e}_{6}$. This result can also be found in Adams' book [2], phrased as follows:

$$
\mathfrak{e}_{6} \cong \mathfrak{s o}(10) \oplus \mathfrak{u}(1) \oplus S_{10} .
$$

In fact, he describes the bracket in $\mathfrak{e}_{6}$ in terms of natural operations involving $\mathfrak{s o}(10)$ and its spinor representation $S_{10}$. The funny-looking factor of $\mathfrak{u}(1)$ comes from the fact that this spinor representation is complex. The bracket of an element of $\mathfrak{u}(1)$ and an element of $S_{10}$ is another element of $S_{10}$, defined using the obvious action of $\mathfrak{u}(1)$ on this complex vector space.

If we define $\mathrm{E}_{6}$ to be the simply connected group with Lie algebra $\mathfrak{e}_{6}$, it follows from results of Adams that the subgroup generated by the Lie subalgebra $\mathfrak{s o}(10) \oplus$ $\mathfrak{u}(1)$ is isomorphic to $(\operatorname{Spin}(10) \times \mathrm{U}(1)) / \mathbb{Z}_{4}$. This lets us define the bioctonionic projective plane by

$$
(\mathbb{C} \otimes \mathbb{O}) \mathbb{P}^{2}=\mathrm{E}_{6} /\left((\operatorname{Spin}(10) \times \mathrm{U}(1)) / \mathbb{Z}_{4}\right)
$$

and conclude that the tangent space at any point of this manifold is isomorphic to $S_{10} \cong(\mathbb{C} \otimes \mathbb{O})^{2}$.

Since $\mathrm{E}_{6}$ is compact, we can put an $\mathrm{E}_{6}$-invariant Riemannian metric on the bioctonionic projective plane by averaging any metric with respect to the action of 
this group. It turns out [6] that the isometry group of this metric is exactly $\mathrm{E}_{6}$, so we have

$$
\mathrm{E}_{6} \cong \operatorname{Isom}\left((\mathbb{C} \otimes \mathbb{O}) \mathbb{P}^{2}\right)
$$

It follows that

$$
\mathfrak{e}_{6} \cong \mathfrak{i s o m}\left((\mathbb{C} \otimes \mathbb{O}) \mathbb{P}^{2}\right) .
$$

Summarizing, we have 6 octonionic descriptions of $\mathfrak{e}_{6}$ :

Theorem 6. The compact real form of $\mathfrak{e}_{6}$ is given by

$$
\begin{aligned}
\mathfrak{e}_{6} & \cong \mathfrak{i s o m}\left((\mathbb{C} \otimes \mathbb{O}) \mathbb{P}^{2}\right) \\
& \cong \operatorname{der}(\mathbb{O}) \oplus \mathfrak{d e r}\left(\mathfrak{h}_{3}(\mathbb{C})\right) \oplus\left(\operatorname{Im}(\mathbb{O}) \otimes \mathfrak{s h} \mathfrak{h}_{3}(\mathbb{C})\right) \\
& \cong \mathfrak{d e r}\left(\mathfrak{h}_{3}(\mathbb{O})\right) \oplus \mathfrak{s h}(\mathbb{O}) \\
& \cong \mathfrak{d e r}(\mathbb{O}) \oplus \mathfrak{s a} \mathfrak{a}_{3}(\mathbb{C} \otimes \mathbb{O}) \\
& \cong \mathfrak{s o}(\mathbb{O} \oplus \mathbb{C}) \oplus \operatorname{Im}(\mathbb{C}) \oplus(\mathbb{C} \otimes \mathbb{O})^{2} \\
& \cong \mathfrak{s o}(\mathbb{O}) \oplus \mathfrak{s o}(\mathbb{C}) \oplus \operatorname{Im}(\mathbb{C}) \oplus(\mathbb{C} \otimes \mathbb{O})^{3}
\end{aligned}
$$

where in each case the Lie bracket of $\mathfrak{e}_{6}$ is built from natural bilinear operations on the summands.

The smallest nontrivial representations of $\mathrm{E}_{6}$ are 27-dimensional: in fact it has two inequivalent representations of this dimension, which are dual to one another. Now, the exceptional Jordan algebra is also 27-dimensional, and in 1950 this clue led Chevalley and Schafer [20] to give a nice description of $\mathrm{E}_{6}$ as symmetries of this algebra. These symmetries do not preserve the product, but only the determinant.

More precisely, the group of determinant-preserving linear transformations of $\mathfrak{h}_{3}(\mathbb{O})$ turns out to be a noncompact real form of $\mathrm{E}_{6}$. This real form is sometimes called $\mathrm{E}_{6(-26)}$, because its Killing form has signature -26 . To see this, note that any automorphism of $\mathfrak{h}_{3}(\mathbb{O})$ preserves the determinant, so we get an inclusion

$$
\mathrm{F}_{4} \hookrightarrow \mathrm{E}_{6(-26)} .
$$

This means that $\mathrm{F}_{4}$ is a compact subgroup of $\mathrm{E}_{6(-26)}$. In fact it is a maximal compact subgroup, since if there were a larger one, we could average a Riemannian metric group on $\mathbb{O P}^{2}$ with respect to this group and get a metric with an isometry group larger than $\mathrm{F}_{4}$, but no such metric exists. It follows that the Killing form on the Lie algebra $\mathfrak{e}_{6(-26)}$ is negative definite on its 52-dimensional maximal compact Lie algebra, $\mathfrak{f}_{4}$, and positive definite on the complementary 26-dimensional subspace, giving a signature of $26-52=-26$.

We saw in Section 3.4 that the projective plane structure of $\mathbb{O P}^{2}$ can be constructed starting only with the determinant function on the vector space $\mathfrak{h}_{3}(\mathbb{O})$. It follows that $\mathrm{E}_{6(-26)}$ acts as collineations on $\mathbb{O P}^{2}$, that is, line-preserving transformations. In fact, the group of collineations of $\mathbb{O P}^{2}$ is precisely $\mathrm{E}_{6(-26)}$ :

$$
\mathrm{E}_{6(-26)} \cong \operatorname{Coll}\left(\mathbb{O P}^{2}\right) \text {. }
$$

Moreover, just as the group of isometries of $\mathbb{O P}^{2}$ fixing a specific point is a copy of $\operatorname{Spin}(9)$, the group of collineations fixing a specific point is $\operatorname{Spin}(9,1)$. This fact follows with some work starting from equation (8), and it gives us a commutative 
square of inclusions:

$$
\begin{array}{ccc}
\operatorname{Spin}(9) & \longrightarrow & \operatorname{Isom}\left(\mathbb{O P}^{2}\right) \cong \mathrm{F}_{4} \\
\downarrow & & \downarrow \\
\operatorname{Spin}(9,1) & \longrightarrow & \operatorname{Coll}\left(\mathbb{O P}^{2}\right) \cong \mathrm{E}_{6(-26)}
\end{array}
$$

where the groups on the top are maximal compact subgroups of those on the bottom. Thus in a very real sense, $\mathrm{F}_{4}$ is to 9-dimensional Euclidean geometry as $\mathrm{E}_{6(-26)}$ is to 10-dimensional Lorentzian geometry.

4.5. $\mathbf{E}_{7}$. Next we turn to the 133-dimensional exceptional Lie group $\mathrm{E}_{7}$. In 1954 , Freudenthal [40] described this group as the automorphism group of a 56-dimensional octonionic structure now called a 'Freudenthal triple system'. We sketch this idea below, but first we give some magic square constructions. Vinberg's version of the magic square gives

$$
\mathfrak{e}_{7}=\mathfrak{d e r}(\mathbb{H}) \oplus \mathfrak{d e r}(\mathbb{O}) \oplus \mathfrak{s a} \mathfrak{a}_{3}(\mathbb{H} \otimes \mathbb{O}) .
$$

Tits' version gives

$$
\mathfrak{e}_{7} \cong \mathfrak{d e r}(\mathbb{H}) \oplus \mathfrak{d e r}\left(\mathfrak{h}_{3}(\mathbb{O})\right) \oplus\left(\operatorname{Im}(\mathbb{H}) \otimes \mathfrak{s h} \mathfrak{h}_{3}(\mathbb{O})\right)
$$

and also

$$
\mathfrak{e}_{7} \cong \mathfrak{d e r}(\mathbb{O}) \oplus \mathfrak{d e r}\left(\mathfrak{h}_{3}(\mathbb{H})\right) \oplus\left(\operatorname{Im}(\mathbb{O}) \otimes \mathfrak{s h} \mathfrak{h}_{3}(\mathbb{H})\right) .
$$

The Barton-Sudbery version gives

$$
\mathfrak{e}_{7} \cong \mathfrak{t r i}(\mathbb{O}) \oplus \mathfrak{t r i}(\mathbb{H}) \oplus(\mathbb{H} \otimes \mathbb{O})^{3} .
$$

Starting from equation (22) and using the fact that $\mathfrak{d e r}(\mathbb{H}) \cong \operatorname{Im}(\mathbb{H})$ is 3 dimensional, we obtain the elegant formula

$$
\mathfrak{e}_{7} \cong \mathfrak{d e r}\left(\mathfrak{h}_{3}(\mathbb{O})\right) \oplus \mathfrak{h}_{3}(\mathbb{O})^{3} .
$$

This gives an illuminating way to compute the dimension of $\mathfrak{e}_{7}$ :

$$
\operatorname{dim}\left(\mathfrak{e}_{7}\right)=\operatorname{dim}\left(\mathfrak{d e r}\left(\mathfrak{h}_{3}(\mathbb{O})\right)\right)+3 \operatorname{dim}\left(\mathfrak{h}_{3}(\mathbb{O})\right)=52+3 \cdot 27=133 .
$$

Starting from equation $(23)$ and using the concrete descriptions of $\operatorname{tri}(\mathbb{H})$ and $\operatorname{tri}(\mathbb{O})$ from equation (21), we obtain

$$
\mathfrak{e}_{7} \cong \mathfrak{s o}(\mathbb{O}) \oplus \mathfrak{s o}(\mathbb{H}) \oplus \operatorname{Im}(\mathbb{H}) \oplus(\mathbb{H} \otimes \mathbb{O})^{3} .
$$

Using equation (17), we may rewrite this as

$$
\mathfrak{e}_{7} \cong \mathfrak{s o}(\mathbb{O} \oplus \mathbb{H}) \oplus \operatorname{Im}(\mathbb{H}) \oplus(\mathbb{H} \otimes \mathbb{O})^{2} .
$$

Though not obvious from what we have done, the direct summand $\mathfrak{s o}(\mathbb{O} \oplus \mathbb{H}) \oplus \operatorname{Im}(\mathbb{H})$ here is really a Lie subalgebra of $\mathfrak{e}_{7}$. In less octonionic language, this result can also be found in Adams' book [2]:

$$
\mathfrak{e}_{7} \cong \mathfrak{s o}(12) \oplus \mathfrak{s p}(1) \oplus S_{12}^{+} .
$$

He describes the bracket in $\mathfrak{e}_{7}$ in terms of natural operations involving $\mathfrak{s o}(12)$ and its spinor representation $S_{12}^{+}$. The funny-looking factor of $\mathfrak{s p}(1)$ comes from the fact that this representation is quaternionic. The bracket of an element of $\mathfrak{s p}(1)$ and an element of $S_{12}^{+}$is the element of $S_{12}^{+}$defined using the natural action of $\mathfrak{s p}(1)$ on this space.

If we let $\mathrm{E}_{7}$ be the simply connected group with Lie algebra $\mathfrak{e}_{7}$, it follows from results of Adams [2] that the subgroup generated by the Lie subalgebra $\mathfrak{s o}(12) \oplus \mathfrak{s} \mathfrak{p}(1)$ 
is isomorphic to $(\operatorname{Spin}(12) \times \operatorname{Sp}(1)) / \mathbb{Z}_{2}$. This lets us define the quateroctonionic projective plane by

$$
(\mathbb{H} \otimes \mathbb{O}) \mathbb{P}^{2}=\mathrm{E}_{7} /\left((\operatorname{Spin}(12) \times \mathrm{Sp}(1)) / \mathbb{Z}_{2}\right)
$$

and conclude that the tangent space at any point of this manifold is isomorphic to $S_{12}^{+} \cong(\mathbb{H} \otimes \mathbb{O})^{2}$. We can put an $\mathrm{E}_{7}$-invariant Riemannian metric on this manifold by the technique of averaging over the group action. It then turns out [6] that

$$
\mathrm{E}_{7} \cong \operatorname{Isom}\left((\mathbb{H} \otimes \mathbb{O}) \mathbb{P}^{2}\right)
$$

and thus

$$
\mathfrak{e}_{7} \cong \mathfrak{i s o m}\left((\mathbb{H} \otimes \mathbb{O}) \mathbb{P}^{2}\right) .
$$

Summarizing, we have the following 7 octonionic descriptions of $\mathfrak{e}_{7}$ :

Theorem 7. The compact real form of $\mathfrak{e}_{7}$ is given by

$$
\begin{aligned}
\mathfrak{e}_{7} & \cong \mathfrak{i s o m}\left((\mathbb{H} \otimes \mathbb{O}) \mathbb{P}^{2}\right) \\
& \cong \mathfrak{d e r}\left(\mathfrak{h}_{3}(\mathbb{O})\right) \oplus \mathfrak{h}_{3}(\mathbb{O})^{3} \\
& \cong \mathfrak{d e r}(\mathbb{O}) \oplus \mathfrak{d e r}\left(\mathfrak{h}_{3}(\mathbb{H})\right) \oplus\left(\operatorname{Im}(\mathbb{O}) \otimes \mathfrak{s h} \mathfrak{h}_{3}(\mathbb{H})\right) \\
& \cong \mathfrak{d e r}(\mathbb{H}) \oplus \mathfrak{d e r}\left(\mathfrak{h}_{3}(\mathbb{O})\right) \oplus\left(\operatorname{Im}(\mathbb{H}) \otimes \mathfrak{s h} \mathfrak{H}_{3}(\mathbb{O})\right) \\
& \cong \mathfrak{d e r}(\mathbb{O}) \oplus \mathfrak{d e r}(\mathbb{H}) \oplus \mathfrak{s a} \mathfrak{a}_{3}(\mathbb{H} \otimes \mathbb{O}) \\
& \cong \mathfrak{s o}(\mathbb{O} \oplus \mathbb{H}) \oplus \operatorname{Im}(\mathbb{H}) \oplus(\mathbb{H} \otimes \mathbb{O})^{2} \\
& \cong \mathfrak{s o}(\mathbb{O}) \oplus \mathfrak{s} \mathfrak{H}(\mathbb{H}) \oplus \operatorname{Im}(\mathbb{H}) \oplus(\mathbb{H} \otimes \mathbb{O})^{3}
\end{aligned}
$$

where in each case the Lie bracket of $\mathfrak{e}_{7}$ is built from natural bilinear operations on the summands.

Before the magic square was developed, Freudenthal 40 used another octonionic construction to study $\mathrm{E}_{7}$. The smallest nontrivial representation of this group is 56-dimensional. Freudenthal realized we can define a 56-dimensional space

$$
F=\left\{\left(\begin{array}{cc}
\alpha & x \\
y & \beta
\end{array}\right): x, y \in \mathfrak{h}_{3}(\mathbb{O}), \alpha, \beta \in \mathbb{R}\right\}
$$

and equip this space with a symplectic structure

$$
\omega: F \times F \rightarrow \mathbb{R}
$$

and trilinear product

$$
\tau: F \times F \times F \rightarrow F
$$

such that the group of linear transformations preserving both these structures is a certain noncompact real form of $\mathrm{E}_{7}$, namely $\mathrm{E}_{7(-25)}$. The symplectic structure and trilinear product on $F$ satisfy some relations, and algebraists have made these into the definition of a 'Freudenthal triple system' [12], 35], [71]. The geometrical significance of this rather complicated sort of structure has recently been clarified by some physicists working on string theory. At the end of the previous section, we mentioned a relation between 9-dimensional Euclidean geometry and $\mathrm{F}_{4}$, and a corresponding relation between 10-dimensional Lorentzian geometry and $\mathrm{E}_{6(-26)}$. Murat Günaydin [47] has extended this to a relation between 10-dimensional conformal geometry and $\mathrm{E}_{7(-25)}$, and in work with Kilian Koepsell and Hermann Nikolai [48] has explicated how this is connected to Freudenthal triple systems. 
4.6. $\mathbf{E}_{8}$. With 248 dimensions, $\mathrm{E}_{8}$ is the biggest of the exceptional Lie groups, and in some ways the most mysterious. The easiest way to understand a group is to realize it as symmetries of a structure one already understands. Of all the simple Lie groups, $\mathrm{E}_{8}$ is the only one whose smallest nontrivial representation is the adjoint representation. This means that in the context of linear algebra, $\mathrm{E}_{8}$ is most simply described as the group of symmetries of its own Lie algebra! One way out of this vicious circle would be to describe $\mathrm{E}_{8}$ as isometries of a Riemannian manifold. As already mentioned, $\mathrm{E}_{8}$ is the isometry group of a 128-dimensional manifold called $(\mathbb{O} \otimes \mathbb{O}) \mathbb{P}^{2}$. But alas, nobody seems to know how to define $(\mathbb{O} \otimes \mathbb{O}) \mathbb{P}^{2}$ without first defining $\mathrm{E}_{8}$. Thus this group remains a bit enigmatic.

At present, to get our hands on $\mathrm{E}_{8}$ we must start with its Lie algebra. We can define this using any of the three equivalent magic square constructions explained in Section 4.3. Vinberg's construction gives

$$
\mathfrak{e}_{8}=\mathfrak{d e r}(\mathbb{O}) \oplus \mathfrak{d e r}(\mathbb{O}) \oplus \mathfrak{s a} \mathfrak{a}_{3}(\mathbb{O} \otimes \mathbb{O}) .
$$

Tits' construction gives

$$
\mathfrak{e}_{8} \cong \mathfrak{d e r}(\mathbb{O}) \oplus \mathfrak{d e r}\left(\mathfrak{h}_{3}(\mathbb{O})\right) \oplus\left(\operatorname{Im}(\mathbb{O}) \otimes \mathfrak{s h} \mathfrak{h}_{3}(\mathbb{O})\right) .
$$

The Barton-Sudbery construction gives

$$
\begin{aligned}
\mathfrak{e}_{8} & \cong \operatorname{tri}(\mathbb{O}) \oplus \mathfrak{t r i}(\mathbb{O}) \oplus(\mathbb{O} \otimes \mathbb{O})^{3} \\
& \cong \mathfrak{s o}(\mathbb{O}) \oplus \mathfrak{s o}(\mathbb{O}) \oplus(\mathbb{O} \otimes \mathbb{O})^{3} .
\end{aligned}
$$

We can use any of these to count the dimension of $\mathfrak{e}_{8}$; for example, the last one gives

$$
\operatorname{dim} \mathfrak{e}_{8}=28+28+3 \cdot 8^{2}=248 .
$$

To emphasize the importance of triality, we can rewrite equation (24) as:

$$
\mathfrak{e}_{8} \cong \mathfrak{s o}(8) \oplus \mathfrak{s o}(8) \oplus\left(V_{8} \otimes V_{8}\right) \oplus\left(S_{8}^{+} \otimes S_{8}^{+}\right) \oplus\left(S_{8}^{-} \otimes S_{8}^{-}\right) .
$$

Here the Lie bracket is built from natural maps relating $\mathfrak{s o}(8)$ and its three 8dimensional irreducible representations. In particular, $\mathfrak{s o}(8) \oplus \mathfrak{s o}(8)$ is a Lie subalgebra, and the first copy of $\mathfrak{s o}(8)$ acts on the first factor in $V_{8} \otimes V_{8}, S_{8}^{+} \otimes S_{8}^{+}$, and $S_{8}^{-} \otimes S_{8}^{-}$, while the second copy acts on the second factor in each of these. The reader should compare this to the description of $\mathfrak{f}_{4}$ in equation (18).

Now, equation (17) implies that

$$
\mathfrak{s o}(16) \cong \mathfrak{s o}(8) \oplus \mathfrak{s o}(8) \oplus\left(V_{8} \otimes V_{8}\right) .
$$

Together with equation (25), this suggests that $\mathfrak{e}_{8}$ contains $\mathfrak{s o}(16)$ as a Lie subalgebra. In fact this is true! Even better, if we restrict the right-handed spinor representation of $\mathfrak{s o}(16)$ to $\mathfrak{s o}(8) \oplus \mathfrak{s o}(8)$, it decomposes as

$$
S_{16}^{+} \cong\left(S_{8}^{+} \otimes S_{8}^{+}\right) \oplus\left(S_{8}^{-} \otimes S_{8}^{-}\right),
$$

so we obtain

$$
\mathfrak{e}_{8} \cong \mathfrak{s o}(16) \oplus S_{16}^{+}
$$

or in more octonionic language,

$$
\mathfrak{e}_{8} \cong \mathfrak{s o}(\mathbb{O} \oplus \mathbb{O}) \oplus(\mathbb{O} \otimes \mathbb{O})^{2}
$$

where we use $\mathfrak{s o}(V)$ to mean the Lie algebra of skew-adjoint real-linear transformations of the real inner product space $V$. 
The really remarkable thing about equation (26) is that the Lie bracket in $\mathfrak{e}_{8}$ is entirely built from natural maps involving $\mathfrak{s o}(16)$ and $S_{16}^{+}$:

$$
\mathfrak{s o}(16) \otimes \mathfrak{s o}(16) \rightarrow \mathfrak{s o}(16), \quad \mathfrak{s o}(16) \otimes S_{16}^{+} \rightarrow S_{16}^{+}, \quad S_{16}^{+} \otimes S_{16}^{+} \rightarrow \mathfrak{s o}(16) .
$$

The first of these is the Lie bracket in $\mathfrak{s o}(16)$, the second is the action of $\mathfrak{s o}(16)$ on its right-handed spinor representation, and the third is obtained from the second by duality, using the natural inner product on $\mathfrak{s o}(16)$ and $S_{16}^{+}$to identify these spaces with their duals. In fact, this is a very efficient way to define $\mathfrak{e}_{8}$. If we take this approach, we must verify the Jacobi identity:

$$
[[a, b], c]]=[a,[b, c]]-[b,[a, c]]
$$

When all three of $a, b, c$ lie in $\mathfrak{s o}(16)$ this is just the Jacobi identity for $\mathfrak{s o}(16)$. When two of them lie in $\mathfrak{s o}(16)$, it boils down to the fact that spinors indeed form a representation of $\mathfrak{s o}(16)$. Thanks to duality, the same is true when just one lies in $\mathfrak{s o}(16)$. It thus suffices to consider the case when $a, b, c$ all lie in $S_{16}^{+}$. This is the only case that uses anything special about the number 16 . Unfortunately, at this point a brute-force calculation seems to be required. For two approaches that minimize the pain, see the books by Adams [2] and by Green, Schwarz and Witten 45]. It would be nice to find a more conceptual approach.

Starting from $\mathfrak{e}_{8}$, we can define $\mathrm{E}_{8}$ to be the simply-connected Lie group with this Lie algebra. As shown by Adams [2], the subgroup of $\mathrm{E}_{8}$ generated by the Lie subalgebra $\mathfrak{s o}(16) \subset \mathfrak{e}_{8}$ is $\operatorname{Spin}(16) / \mathbb{Z}_{2}$. This lets us define the octooctonionic projective plane by

$$
(\mathbb{O} \otimes \mathbb{O}) \mathbb{P}^{2}=\mathrm{E}_{8} /\left(\operatorname{Spin}(16) / \mathbb{Z}_{2}\right)
$$

By equation (26), the tangent space at any point of this manifold is isomorphic to $S_{16}^{+} \cong(\mathbb{O} \otimes \mathbb{O})^{2}$. This partially justifies calling it the 'octooctonionic projective plane', though it seems not to satisfy the usual axioms for a projective plane.

We can put an $\mathrm{E}_{8}$-invariant Riemannian metric on the octooctonionic projective plane by the technique of averaging over the group action. It then turns out 6 ] that

$$
\mathrm{E}_{8} \cong \operatorname{Isom}\left((\mathbb{O} \otimes \mathbb{O}) \mathbb{P}^{2}\right)
$$

and thus

$$
\mathfrak{e}_{8} \cong \mathfrak{i s o m}\left((\mathbb{O} \otimes \mathbb{O}) \mathbb{P}^{2}\right)
$$

Summarizing, we have the following octonionic descriptions of $\mathrm{E}_{8}$ :

Theorem 8. The compact real form of $\mathfrak{e}_{8}$ is given by

$$
\begin{aligned}
\mathfrak{e}_{8} & \cong \mathfrak{i s o m}\left((\mathbb{O} \otimes \mathbb{O}) \mathbb{P}^{2}\right) \\
& \cong \mathfrak{d e r}(\mathbb{O}) \oplus \mathfrak{d e r}\left(\mathfrak{h}_{3}(\mathbb{O})\right) \oplus\left(\operatorname{Im}(\mathbb{O}) \otimes \mathfrak{s h} \mathfrak{h}_{3}(\mathbb{O})\right) \\
& \cong \mathfrak{d e r}(\mathbb{O}) \oplus \mathfrak{d e r}(\mathbb{O}) \oplus \mathfrak{s a} \mathfrak{a}_{3}(\mathbb{O} \otimes \mathbb{O}) \\
& \cong \mathfrak{s o}(\mathbb{O} \oplus \mathbb{O}) \oplus(\mathbb{O} \otimes \mathbb{O})^{2} \\
& \cong \mathfrak{s o}(\mathbb{O}) \oplus \mathfrak{s o}(\mathbb{O}) \oplus(\mathbb{O} \otimes \mathbb{O})^{3}
\end{aligned}
$$

where in each case the Lie bracket on $\mathfrak{e}_{8}$ is built from natural bilinear operations on the summands. 


\section{Conclusions}

It should be clear by now that besides being a fascinating mathematical object in their own right, the octonions link together many important phenomena whose connections would otherwise be completely mysterious. Indeed, the full story of these connections is deeper and more elaborate than I have been able to explain here! It also includes:

- Attempts to set up an octonionic analogue of the theory of analytic functions (see [50] and the references therein).

- The role of Jordan pairs, Jordan triple systems and Freudenthal triple systems in the construction of exceptional Lie groups [12, 35], 36], 48, 50], [70, 71].

- Constructions of the $\mathrm{E}_{8}$ lattice and Leech lattice using integral octonions [25], 32.

- Tensor-categorical approaches to normed division algebras and the invariant of framed trivalent graphs coming from the quantum group associated to $\mathrm{G}_{2}$ [9], 64, 80].

- Octonionic constructions of vertex operator algebras [37].

- Octonionic constructions of the exceptional simple Lie superalgebras [91].

- Octonionic constructions of symmetric spaces [6].

- Octonions and the geometry of the 'squashed 7-spheres', that is, the homogeneous spaces $\operatorname{Spin}(7) / \mathrm{G}_{2}, \operatorname{Spin}(6) / \mathrm{SU}(3)$, and $\operatorname{Spin}(5) / \mathrm{SU}(2)$, all of which are diffeomorphic to $S^{7}$ with its usual smooth structure 21].

- The theory of 'Joyce manifolds', that is, 7-dimensional Riemannian manifolds with holonomy group $\mathrm{G}_{2}$ [59].

- The octonionic Hopf map and instanton solutions of the Yang-Mills equations in 8 dimensions [46].

- Octonionic aspects of 10-dimensional superstring theory and 10-dimensional super-Yang-Mills theory [24], 28, [34, 63], 84], 85].

- Octonionic aspects of 11-dimensional supergravity and supermembrane theories, and the role of Joyce manifolds in compactifying 11-dimensional supergravity to obtain theories of physics in 4 dimensions 31 .

- Geoffrey Dixon's extension of the Standard Model based on the algebra $\mathbb{C} \otimes$ $\mathbb{H} \otimes \mathbb{O}[30]$.

- Other attempts to use the octonions in physics [19], [50, 66], [74].

I urge the reader to explore these with the help of the references.

Acknowledgements. I thank John Barrett, Toby Bartels, Robert Bryant, Geoffrey Dixon, James Dolan, Tevian Dray, Bertram Kostant, Linus Kramer, Pertti Lounesto, Corinne Manogue, John McKay, David Rusin, Tony Smith, Anthony Sudbery, and Matthew Wiener for useful discussions.

\section{REFERENCES}

1. John F. Adams, On the non-existence of elements of Hopf invariant one, Ann. Math. 72 (1960), 20-104. MR 25:4530

2. John F. Adams, Lectures on Exceptional Lie Groups, eds. Zafer Mahmud and Mamoru Mimira, University of Chicago Press, Chicago, 1996. MR 98b:22001

3. Michael Atiyah and Friedrich Hirzebruch, Bott periodicity and the parallelizability of the spheres. Proc. Cambridge Philos. Soc. 57 (1961), 223-226. MR 23:A3578 
4. Helena Albuquerque and Shahn Majid, Quasialgebra structure of the octonions, J. Algebra 220 (1999), 188-224. MR 2000h:16048

5. Chris H. Barton and Anthony Sudbery, Magic squares of Lie algebras, preprint available as math.RA/0001083.

6. Arthur L. Besse, Einstein Manifolds, Springer, Berlin, 1987, pp. 313-316. MR 88f:53087

7. F. van der Blij, History of the octaves, Simon Stevin 34 (1961), 106-125. MR 24:A149

8. F. van der Blij and Tonny A. Springer, Octaves and triality, Nieuw Arch. v. Wiskunde 8 (1960), 158-169. MR 23:A947

9. Dominik Boos, Ein tensorkategorieller Zugang zum Satz von Hurwitz, Diplomarbeit, ETH Zurich, March 1998.

10. Armand Borel, Le plan projectif des octaves et les sphéres commes espaces homogènes, Compt. Rend. Acad. Sci. 230 (1950), 1378-1380. MR 11:640c

11. Raoul Bott and John Milnor, On the parallelizability of the spheres, Bull. Amer. Math. Soc. 64 (1958), 87-89. MR 21:1590]

12. Robert B. Brown, Groups of type $\mathrm{E}_{7}$, Jour. Reine Angew. Math. 236 (1969), 79-102. MR 40:1439

13. Élie Cartan, Sur la structure des groupes de tranformations finis et continus, Thèse, Paris, Nony, 1894.

14. Élie Cartan, Les groupes réels simples finis et continus, Ann. Sci. École Norm. Sup. 31 (1914), 255-262.

15. Élie Cartan, Nombres complexes, in Encyclopédie des sciences mathématiques, 1, ed. J. Molk, 1908, 329-468.

16. Élie Cartan, Le principe de dualité et la théorie des groupes simple et semi-simples, Bull. Sci. Math. 49 (1925), 361-374.

17. Arthur Cayley, On Jacobi's elliptic functions, in reply to the Rev. B. Bronwin; and on quaternions, Philos. Mag. 26 (1845), 208-211.

18. Arthur Cayley, On Jacobi's elliptic functions, in reply to the Rev. B. Bronwin; and on quaternions (appendix only), in The Collected Mathematical Papers, Johnson Reprint Co., New York, 1963, p. 127.

19. Sultan Catto, Carlos J. Moreno and Chia-Hsiung Tze, Octonionic Structures in Physics, to appear.

20. Claude Chevalley and Richard D. Schafer, The exceptional simple Lie algebras $\mathrm{F}_{4}$ and $\mathrm{E}_{6}$, Proc. Nat. Acad. Sci. USA 36 (1950), 137-141. MR 11:577b

21. Yvonne Choquet-Bruhat and Cécile DeWitt-Morette, Analysis, Manifolds and Physics, part II, Elsevier, Amsterdam, 2000, pp. 263-274. MR 91e:58001

22. William K. Clifford, Applications of Grassmann's extensive algebra, Amer. Jour. Math. 1 (1878), 350-358.

23. Frederick R. Cohen, On Whitehead squares, Cayley-Dickson algebras and rational functions, Bol. Soc. Mat. Mexicana 37 (1992), 55-62. MR 95m:55018

24. E. Corrigan and T. J. Hollowood, The exceptional Jordan algebra and the superstring, Comm. Math. Phys. 122 (1989), 393-410. MR 90j:81183

25. Harold Scott MacDonald Coxeter, Integral Cayley numbers, Duke Math. Jour. 13 (1946), 561-578. MR 8:370b

26. Michael J. Crowe, A History of Vector Analysis, University of Notre Dame Press, Notre Dame, 1967. MR 37:5070

27. C. W. Curtis, The four and eight square problem and division algebras, in Studies in Modern Algebra, ed. A. Albert, Prentice-Hall, Englewood Cliffs, New Jersey, 1963, pp. 100-125.

28. Pierre Deligne et al., eds., Quantum Fields and Strings: A Course for Mathematicians, 2 volumes, Amer. Math. Soc., Providence, Rhode Island, 1999. MR 2000e:81010

29. Leonard E. Dickson, On quaternions and their generalization and the history of the eight square theorem, Ann. Math. 20 (1919), 155-171.

30. Geoffrey M. Dixon, Division Algebras: Octonions, Quaternions, Complex Numbers and the Algebraic Design of Physics, Kluwer, Dordrecht, 1994. MR 96e:17004

31. M. J. Duff, ed., The World in Eleven Dimensions: Supergravity, Supermembranes and MTheory, Institute of Physics Publishing, Bristol, 1999. MR 2001b:81002

32. Noam Elkies and Benedict H. Gross, The exceptional cone and the Leech lattice, Internat. Math. Res. Notices 14 (1996), 665-698. MR 97g:11070 
33. Gerard G. Emch, Algebraic Methods in Statistical Mechanics and Quantum Field Theory, Wiley-Interscience, New York, 1972.

34. J. M. Evans, Supersymmetric Yang-Mills theories and division algebras, Nucl. Phys. B298 (1988), 92-108. MR 89f:81096

35. John R. Faulkner, A construction of Lie algebras from a class of ternary algebras, Trans. Amer. Math. Soc. 155 (1971), 397-408. MR 45:3494

36. John R. Faulkner and Joseph C. Ferrar, Exceptional Lie algebras and related algebraic and geometric structures, Bull. London Math. Soc. 9 (1977), 1-35. MR 56:3079

37. Alex J. Feingold, Igor B. Frenkel, and John F. X. Ries, Spinor Construction of Vertex Operator Algebras, Triality, and $\mathrm{E}_{8}^{(1)}$, Contemp. Math. 121, Amer. Math. Soc., Providence, Rhode Island, 1991. MR 92k:17041

38. Hans Freudenthal, Oktaven, Ausnahmegruppen und Oktavengeometrie, mimeographed notes, 1951. Also available in Geom. Dedicata 19 (1985), 7-63. MR 86k:17018b

39. Hans Freudenthal, Zur ebenen Oktavengeometrie, Indag. Math. 15 (1953), 195-200. MR 15:56f

40. Hans Freudenthal, Beziehungen der $\mathfrak{e}_{7}$ und $\mathfrak{e}_{8}$ zur Oktavenebene, I, II, Indag. Math. 16 (1954), 218-230, 363-368. MR 16:108b MR 16:900d III, IV, Indag. Math. 17 (1955), 151-157, 277285. MR 16:900e MR 16:900f; V-IX, Indag. Math. 21 (1959), 165-201, 447-474; X, XI, Indag. Math. 25 (1963), 457-487. MR 29:506

41. Hans Freudenthal, Lie groups in the foundations of geometry, Adv. Math. 1 (1964), 145-190.

42. Hans Freudenthal, Bericht über die Theorie der Rosenfeldschen elliptischen Ebenen, in Raumtheorie, Wege Der Forschung, CCLXX, Wissenschaftliche Buchgesellschaft, Darmstadt, 1978, pp. 283-286.

43. Lynn E. Garner, An Outline of Projective Geometry, North Holland, New York, 1981. MR 82j:51001

44. Robert Perceval Graves, Life of Sir William Rowan Hamilton, 3 volumes, Arno Press, New York, 1975

45. Michael B. Green, John H. Schwarz and Edward Witten, Superstring Theory, volume 1, Cambridge University Press, Cambridge, 1987, pp. 344-349. MR 89f:81001a

46. B. Grossman, T. E. Kephart, and James D. Stasheff, Solutions to Yang-Mills field equations in eight dimensions and the last Hopf map, Comm. Math. Phys. 96 (1984), 431-437. MR 87b:53048

47. Murat Günaydin, Generalized conformal and superconformal group actions and Jordan algebras, Mod. Phys. Lett. A 8 (1993), 1407-1416. MR 94c:17048

48. Murat Günaydin, Kilian Koepsell, and Hermann Nicolai, Conformal and quasiconformal realizations of exceptional Lie groups, Comm. Math. Phys. 221 (2001), 57-76 CMP 2001:16

49. Murat Günaydin, C. Piron and H. Ruegg, Moufang plane and octonionic quantum mechanics, Comm. Math. Phys. 61 (1978), 69-85. MR 58:19906

50. Feza Gürsey and Chia-Hsiung Tze, On the Role of Division, Jordan, and Related Algebras in Particle Physics, World Scientific, Singapore, 1996. MR 99g:81061

51. William Rowan Hamilton, Four and eight square theorems, in Appendix 3 of The Mathematical Papers of William Rowan Hamilton, vol. 3, eds. H. Halberstam and R. E. Ingram, Cambridge University Press, Cambridge, 1967, pp. 648-656.

52. Thomas L. Hankins, Sir William Rowan Hamilton, Johns Hopkins University Press, Baltimore, 1980. MR 82h:01051

53. F. Reese Harvey, Spinors and Calibrations, Academic Press, Boston, 1990. MR 91e:53056

54. Adolf Hurwitz, Über die Composition der quadratischen Formen von beliebig vielen Variabeln, Nachr. Ges. Wiss. Göttingen (1898), 309-316.

55. Dale Husemoller, Fibre Bundles, Springer, Berlin, 1994. MR 94k:55001

56. Pascual Jordan, Über eine Klasse nichtassociativer hyperkomplexer Algebren, Nachr. Ges. Wiss. Göttingen (1932), 569-575.

57. Pascual Jordan, Über eine nicht-desarguessche ebene projektive Geometrie, Abh. Math. Sem. Hamburg 16 (1949), 74-76. MR 11:50h

58. Pascual Jordan, John von Neumann, Eugene Wigner, On an algebraic generalization of the quantum mechanical formalism, Ann. Math. 35 (1934), 29-64.

59. Dominic Joyce, Compact Manifolds with Special Holonomy, Oxford U. Press, Oxford, 2000. MR 2001k:53093 
60. I. L. Kantor and A. S. Solodovnikov, Hypercomplex Numbers - an Elementary Introduction to Algebras, Springer, Berlin, 1989. MR 90b:16001]

61. Michel Kervaire, Non-parallelizability of the $n$ sphere for $n>7$, Proc. Nat. Acad. Sci. USA 44 (1958), 280-283.

62. Wilhelm Killing, Die Zusammensetzung der stetigen endlichen Transformationsgruppen I, Math. Ann. 31 (1888), 252-290. II, 33 (1889) 1-48. III, 34 (1889), 57-122. IV, 36 (1890), $161-189$.

63. T. Kugo and P.-K. Townsend, Supersymmetry and the division algebras, Nucl. Phys. B 221 (1983), 357-380. MR 85e:81054

64. Greg Kuperberg, Spiders for rank 2 Lie algebras, Comm. Math. Phys. 180 (1996), 109-151. MR 97f: 17005

65. J. M. Landsberg and L. Manivel, The projective geometry of Freudenthal's magic square, J. Algebra 239 (2001), 477-512.

66. Jaak Lohmus, Eugene Paal, and Leo Sorgsepp, Nonassociative Algebras in Physics, Hadronic Press, Palm Harbor, Florida, 1994. MR 97d:81095

67. Corinne A. Manogue and Tevian Dray, Octonionic Möbius transformations, Mod. Phys. Lett. A 14 (1999), 1243-1256. MR 2000g:17003

68. Corinne A. Manogue and Jörg Schray, Finite Lorentz transformations, automorphisms, and division algebras, Jour. Math. Phys. 34 (1993), 3746-3767. MR 94h:81041

69. Corinne A. Manogue and Jörg Schray, Octonionic representations of Clifford algebras and triality, Found. Phys. 26 (1996), 17-70. MR 97d:15035

70. Kevin McCrimmon, Jordan algebras and their applications, Bull. Amer. Math. Soc. 84 (1978), 612-627. MR 57:6115

71. K. Meyberg, Eine Theorie der Freudenthalschen Tripelsysteme, I, II, Indag. Math. 30 (1968), 162-190. MR 37:1429

72. R. Guillermo Moreno, The zero divisors of the Cayley-Dickson algebras over the real numbers, preprint available at q-alg/9710013.

73. Ruth Moufang, Alternativkörper und der Satz vom vollständigen Vierseit, Abh. Math. Sem. Hamburg 9 (1933), 207-222.

74. Susumu Okubo, Introduction to Octonion and Other Non-Associative Algebras in Physics, Cambridge University Press, Cambridge, 1995. MR 96j:81052

75. A. L. Onishchik and E. B. Vinberg, eds., Lie Groups and Lie Algebras III, Springer, Berlin, 1991, pp. 167-178. MR 96d:22001

76. Roger Penrose and Wolfgang Rindler, Spinors and Space-Time, 2 volumes, Cambridge U. Press, Cambridge, 1985-86. MR 88h:83009 MR 89d:83010

77. Ian R. Porteous, Topological Geometry, Cambridge U. Press, 1981. MR 82c:51018

78. Boris A. Rosenfeld, Geometrical interpretation of the compact simple Lie groups of the class E (Russian), Dokl. Akad. Nauk. SSSR 106 (1956), 600-603.

79. Boris A. Rosenfeld, Geometry of Lie Groups, Kluwer, Dordrecht, 1997. MR 98i:53002

80. Markus Rost, On the dimension of a composition algebra, Doc. Math. 1 (1996), 209-214. MR 97c:17004

81. Helmut Salzmann et al., Compact Projective Planes: With an Introduction to Octonion Geometry, de Gruyter, Berlin, 1995. MR 97b:51009

82. Richard D. Schafer, On algebras formed by the Cayley-Dickson process, Amer. Jour. of Math. 76 (1954), 435-446. MR 15:774d

83. Richard D. Schafer, Introduction to Non-Associative Algebras, Dover, New York, 1995. MR 96j:17001

84. Jörg Schray, Octonions and Supersymmetry, Ph.D. thesis, Department of Physics, Oregon State University, 1994.

85. G. Sierra, An application of the theories of Jordan algebras and Freudenthal triple systems to particles and strings, Class. Quant. Grav. 4 (1987), 227-236. MR 88h:81059

86. Tonny A. Springer, The projective octave plane, I-II, Indag. Math. 22 (1960), 74-101. MR 23:A3492

87. Tonny A. Springer, Characterization of a class of cubic forms, Indag. Math. 24 (1962), 259265. MR 25:2104]

88. Tonny A. Springer, On the geometric algebra of the octave planes, Indag. Math. 24 (1962), 451-468. MR 25:5439 
89. Tonny A. Springer and Ferdinand D. Veldkamp, Octonions, Jordan Algebras and Exceptional Groups, Springer, Berlin, 2000. MR 2001f:17006

90. Frederick W. Stevenson, Projective Planes, W. H. Freeman and Company, San Francisco, 1972. MR 49:9734

91. Anthony Sudbery, Octonionic description of exceptional Lie superalgebras, Jour. Math. Phys. 24 (1983), 1986-1988. MR 85c:17011

92. Anthony Sudbery, Division algebras, (pseudo)orthogonal groups and spinors, Jour. Phys. A17 (1984), 939-955. MR 85i:22042

93. Jacques Tits, Le plan projectif des octaves et les groupes de Lie exceptionnels, Bull. Acad. Roy. Belg. Sci. 39 (1953), 309-329. MR 14:947f

94. Jacques Tits, Le plan projectif des octaves et les groupes exceptionnels $\mathrm{E}_{6}$ et $\mathrm{E}_{7}$, Bull. Acad. Roy. Belg. Sci. 40 (1954), 29-40. MR 16:119

95. Jacques Tits, Algèbres alternatives, algèbres de Jordan et algèbres de Lie exceptionnelles, Indag. Math. 28 (1966), 223-237. MR 36:2658

96. Jacques Tits, Buildings of Spherical Type and Finite BN-Pairs, Lecture Notes in Mathematics, Vol. 386, Springer, Berlin, 1974. MR 57:9866

97. V. S. Varadarajan, Geometry of Quantum Theory, Springer-Verlag, Berlin, 1985. MR 87a: 81009

98. E. B. Vinberg, A construction of exceptional simple Lie groups (Russian), Tr. Semin. Vektorn. Tensorn. Anal. 13 (1966), 7-9.

99. Max Zorn, Theorie der alternativen Ringe, Abh. Math. Sem. Univ. Hamburg 8 (1930), 123147.

100. Max Zorn, Alternativkörper und quadratische Systeme, Abh. Math. Sem. Univ. Hamburg 9 (1933), 395-402.

Department of Mathematics, University of California, Riverside, CA 92521

E-mail address: baez@math.ucr.edu 